\title{
On the Security of LEO Satellite Communication Systems: Vulnerabilities, Countermeasures, and Future Trends
}

This paper was downloaded from TechRxiv (https://www.techrxiv.org).

\section{LICENSE}

CC BY-NC-SA 4.0

SUBMISSION DATE / POSTED DATE

$10-01-2022$ / 11-01-2022

\section{CITATION}

Yue, Pingyue; An, Jianping; Zhang, Jiankang; Pan, Gaofeng; Wang, Shuai; Xiao, Pei; et al. (2022): On the Security of LEO Satellite Communication Systems: Vulnerabilities, Countermeasures, and Future Trends. TechRxiv. Preprint. https://doi.org/10.36227/techrxiv.18093941.v1

$\mathrm{DOI}$

10.36227/techrxiv.18093941.v1 


\title{
On the Security of LEO Satellite Communication Systems: Vulnerabilities, Countermeasures, and Future Trends
}

\author{
Pingyue Yue, Student Member, IEEE, Jianping An, Jiankang Zhang, Member, IEEE, \\ Gaofeng Pan, Senior Member, IEEE, Shuai Wang, Member, IEEE, \\ Pei Xiao, Senior Member, IEEE, and Lajos Hanzo, Life Fellow, IEEE
}

\begin{abstract}
Low Earth Orbit (LEO) satellite systems undergo a period of rapid development driven by the ever-increasing user demands, reduced costs, and technological progress. Since there is a paucity of literature on the security issues of LEO Satellite Communication Systems (SCSs), we aim for filling this knowledge gap. Specifically, we critically appraise the inherent characteristics of LEO SCSs and summarize their unique security vulnerabilities. In light of this, we further discuss their security vulnerabilities, including the issues of passive and active eavesdropping attacks, interference scenarios, single event upsets, and space debris. Subsequently, we discuss the corresponding active and passive security countermeasures, followed by unveiling a range of trade-offs, security vulnerabilities and their countermeasures. Furthermore, we shed light on several promising future research directions for enhancing the security of LEO SCSs, such as secure quantum communications, three-dimensional virtual arrays, artificial intelligence-based security measures, spacebased blockchain, and intelligent reflecting surface enabled secure transmission. Finally, the take-away messages of this paper are crystallized in our concluding design guidelines.
\end{abstract}

Index Terms-Active eavesdropping, interference, LEO SCS, passive eavesdropping, security countermeasures, security vulnerabilities, single event upsets, space debris.

\section{INTRODUCTION}

\section{A. Brief review}

Since the early 1980s, there has been a boom in launching small satellites across the globe. Explicitly, by the end of 2021, more than 4700 Low Earth Orbit (LEO) satellites have been successfully launched, accounting for nearly $86 \%$ of the total launch volume of all types of satellites [1]. During these years, LEO Satellite Communication Systems (SCSs) have found a plethora of applications, including media broadcasting,

Pingyue Yue is with the School of Information and Electronics, Beijing Institute of Technology, Beijing 100081, China (e-mails: ypy@bit.edu.cn).

Jianping An (Corresponding author), Gaofeng Pan, and Shuai Wang are with the School of Cyberspace Science and Technology, Beijing Institute of Technology, Beijing 100081, China (e-mails: an@bit.edu.cn; gaofengpan.cn@ieee.org; swang@bit.edu.cn).

Jiankang Zhang is with the Department of Computing and Informatics, Bournemouth University, Bournemouth BH12 5BB, U.K. (e-mail: jzhang3@bournemouth.ac.uk).

Pei Xiao is with the 5GIC \& 6GIC, Institute for Communication Systems, University of Surrey, GU2 7XH, U.K. (e-mail: p.xiao@surrey.ac.uk).

Lajos Hanzo is with the School of Electronics and Computer Science, University of Southampton, Southampton SO17 1BJ, U.K. (e-mail: lh@ecs.soton.ac.uk). backhauling, mobile communication, and broadband Internet [2]-[5]. In fact, LEO SCSs are capable of filling the coverage holes of terrestrial systems and at the time of writing, they tend to evolve towards a converged system, as shown in Fig. 1.

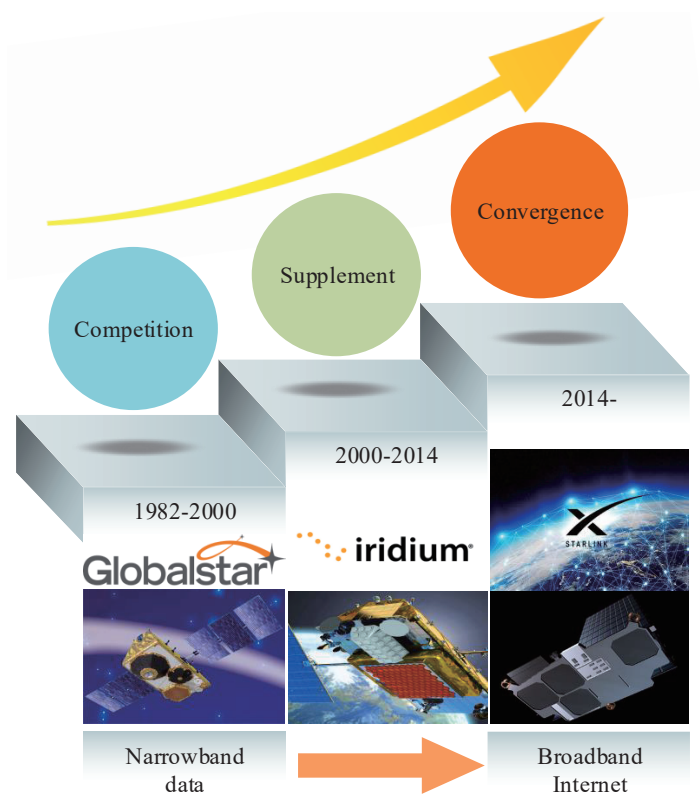

Fig. 1: Development process of LEO SCSs.

From the 1980 s to 2000 , some companies conceived satellite constellation plans. Specifically, Iridium [6], GlobalStar [7], and Orbcomm [8] aspired to build a global LEO constellation for low-speed communication and to sell their satellite communication terminals, so as to attract more users in their competition with terrestrial systems. However, they failed to construct a viable business case owing to their excessive initial investment and a limited number of users [9].

Nevertheless, from 2000 to 2014, Iridium Next [10], GlobalStar, and Orbcomm provided uninterrupted mobile communication services for high-end users roaming in extreme desert, open sea, and aviation scenarios that were not covered by terrestrial systems. Nevertheless, the limited revenues affected their wide-spread development [11].

However, as a benefit of the ever-increasing demands [12], reduced costs [13], and technological progress [14], LEO mega-constellations, such as OneWeb, Starlink, Lightspeed, 
are making a renewed effort for providing service for 'the other 3 Billion' who do not as yet have access to the Internet. They are also capable of eminently suitable for providing backhaul for terrestrial systems. These mega-constellations consist of a large number of LEO satellites supporting communications up to Gbps rates as well as a low delay of tens of milliseconds (ms) [15]. Hence, integrating LEO satellites and terrestrial systems is one of connectivity's new frontiers [16]. The basic features of a range of mega LEO SCSs are summarized in Table I.

\section{B. Serious Security Challenges}

Although the development of LEO SCSs is in full swing, mitigating their security vulnerabilities is set to increase in importance. One of the most significant weaknesses that are common to all SCSs is the use of long-range telemetry for their communication with gateways. Hence SCSs are vulnerable to both eavesdropping and malicious jamming, as it was demonstrated by eavesdropping on the Iridium network via a Software Defined Radio (SDR) device in [26]. A spokesman of the Saudi satellite network said that its Egyptian subsidiary was deliberately jammed when playing a comedy. The satellite operator identified small satellite transmitters in a pair of locations in Cairo as the source of this jamming [27].

Satellites are usually regarded as high-value Internet of Things (IoT) devices, hence they are also vulnerable to hijacking. For a few hundred dollars, hackers can set up a sophisticated high-powered antenna to target and hijack a satellite. Hackers were reported to have controlled SkyNet satellites to extort ransom [28]. Russian hackers previously hijacked commercial satellites to siphon sensitive data from diplomatic and military agencies both in the United States and in Europe [29]. If these compromised satellites are shut down by hackers or even attack other expensive satellites, this might lead to colossal political and financial loss [30].

The space environment is also harsh and each space mission is fraught with challenges. Many aircraft have failed before accomplishing their missions. Cosmic radiation can heavily affect electronic devices [31]. One of the most common effects is the so-called Single Event Upsets (SEUs), which refers to the response of a integrated circuit to a single radiation spin that may cause a temporary failure or a change of state for the IC.

However, the inherent characteristics make LEO SCSs suffer from even more serious security challenges, as shown in Fig. 2.

Given the ongoing deployment of dense LEO megaconstellations, their orbit is becoming increasingly overcrowded. At the same time, more and more spacecraft have become abandoned as well, which poses threats for LEO satellites in-orbit [32]. Additionally, the dramatic increase in the number of spacecraft will undoubtedly increase the probability of collisions.

Spectrum is important for SCSs, because the bandwidth and the propagation properties of the spectrum made available as well as the usage conditions determine both the capacity and the coverage quality, hence ultimately affecting the commercial viability of the system. The spectrum crunch problem due to the scarcity of radio resources results in inevitable spectrum coexistence between SCSs and terrestrial systems. Moreover, the specific location of LEO satellites may also lead to interference with Geostationary Earth Orbit (GEO) SCSs. Typically, a large number of LEO satellites are usually deployed at orbital altitudes of 160 to 2000 kilometers $(\mathrm{km})$, sandwiched between terrestrial systems and GEO SCSs. Severe Co-channel Interference (CCI) may arise whenever LEO satellites pass through the Line of Sight (LoS) path of a GEO satellite in spectral coexistence scenarios. The CCI between LEO satellites and terrestrial systems should also be dealt with in Space-air-ground Integrated Network (SAGIN) [33]. In addition, frequent launch activities have caused a surge in LEO space debris, which poses severe challenges for the operation of LEO satellites.

In contrast to GEO satellites, LEO satellites move at a high speed relative to the Earth. The high mobility of LEO satellites leads to frequent handovers among beams and satellites, which makes the security issues more complex. Frequent handovers require frequent authentication and security key handovers. Illegal users may imitate legitimate users with criminal intent [34], [35].

Again, the high mobility leads to severe Doppler effects, which may significantly deteriorate the system performance. Hence the authors of [36] derived specific expressions for characterizing the Doppler frequency shift, which may be beneficially exploited by sophisticated compensation techniques for maintaining reliable communication.

The weight of LEO satellites usually does not exceed 1000 kilogram $(\mathrm{kg})$ [37]. Hence LEO satellites have limited computing power and storage, which precludes the use of complex security algorithms designed for conventional terrestrial systems. Traditional encryption-based authentication cannot be directly applied to space-borne payloads. As a result, the LEO satellite's space-borne payload typically relies on lowcomplexity security measures operated at the physical-layer.

With the continuous deployment of mega-constellations, the number of LEO satellites has surged, hence it is a challenge to manage a large number of high-speed flying 'base stations'. With more satellites than ever in space, there are more attack opportunities for hackers to compromise the nodes and to threaten normally operating LEO satellites.

As a further potential issue, a large number of lowspecification components used for LEO satellites are supplied by civilian manufacturers both for cost savings, and for reducing the production cycle. For instance, OneWeb is known as another pioneer in the mass-production of satellites, whose satellite factory is in Florida and will produce two satellites per day [13]. However, loopholes in production methods and inadequate testing may lead to potential defects in satellites.

\section{Related Contributions}

Given the pivotal significance of the security, there is a plethora of technical papers aiming for tackling the aforementioned security vulnerabilities. The timeline evolving from 2019 is seen in Fig. 3. 


\begin{tabular}{|c|c|c|c|c|c|c|c|c|c|}
\hline 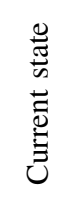 & 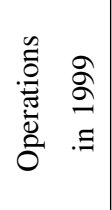 & 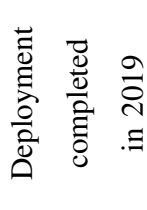 & 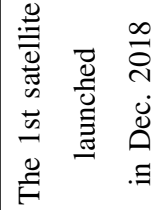 & 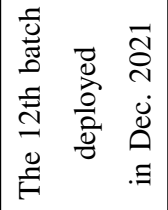 & 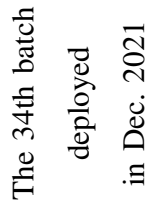 & 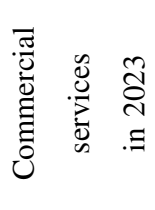 & 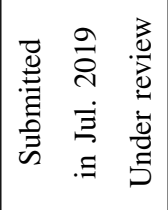 & 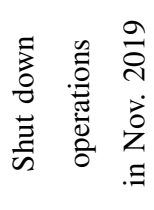 & 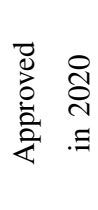 \\
\hline 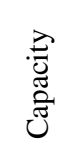 & 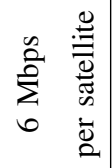 & 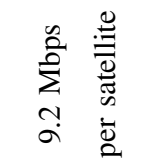 & & 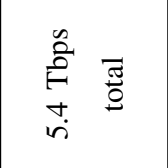 & 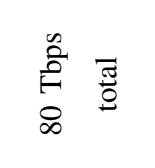 & 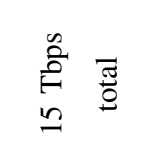 & & 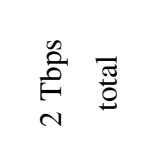 & \\
\hline 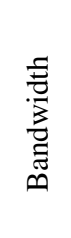 & 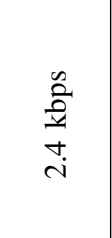 & 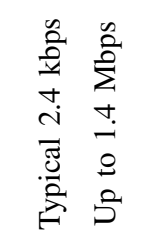 & & 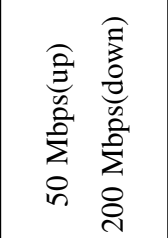 & 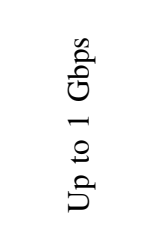 & 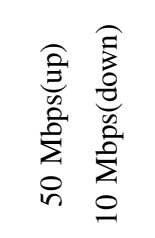 & & 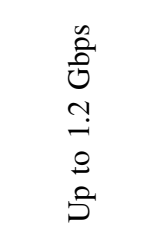 & $\begin{array}{l}0 \\
\sum_{2} \\
8 \\
o \\
o \\
o\end{array}$ \\
\hline 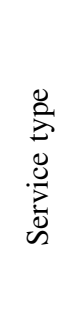 & 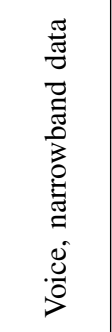 & 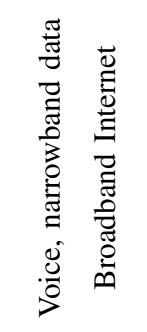 & 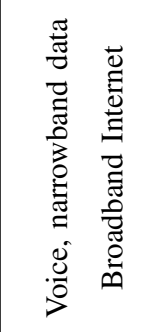 & 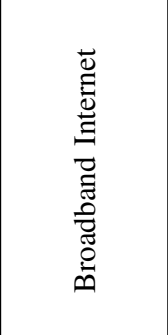 & 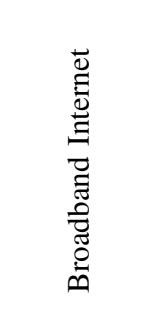 & 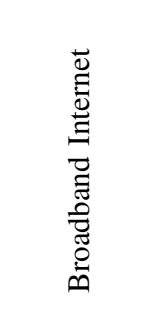 & 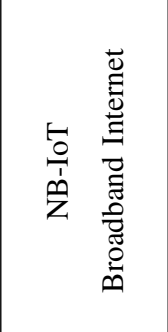 & 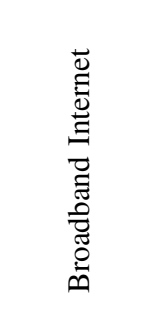 & 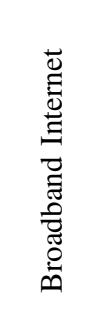 \\
\hline 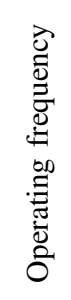 & 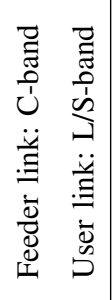 & 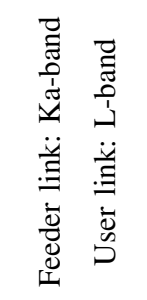 & 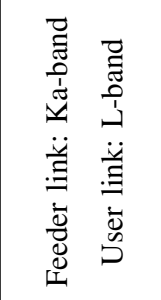 & 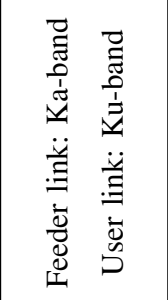 & 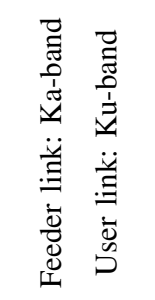 & 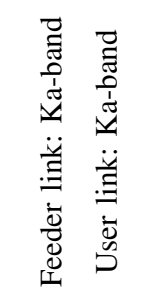 & 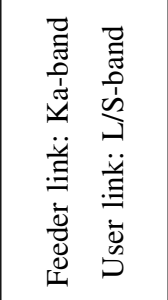 & 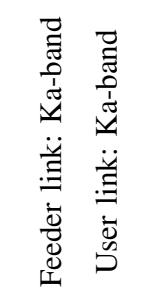 & 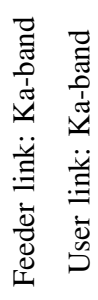 \\
\hline 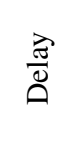 & 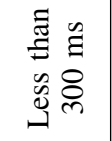 & 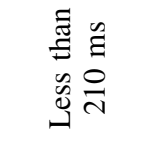 & 1 & 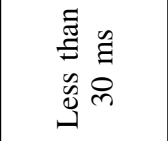 & 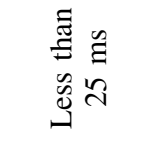 & 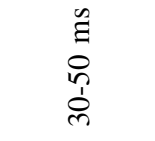 & & & \\
\hline$\hat{\tilde{\Omega}}$ & 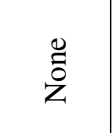 & 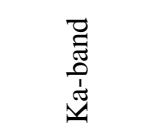 & 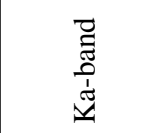 & $\begin{array}{l}\ddot{\Xi} \\
\text { Z }\end{array}$ & 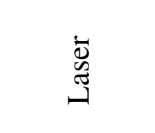 & 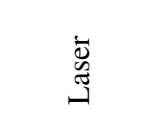 & 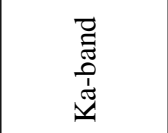 & $\begin{array}{l}\bar{\Xi} \\
\tilde{\Xi} \\
\tilde{J}\end{array}$ & 气 \\
\hline 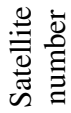 & $\stackrel{\infty}{+}$ & $\bar{\infty}$ & స్ల & $\stackrel{\mathbb{N}}{ }$ & $\widehat{\widehat{\sigma}}$ & $\stackrel{\infty}{\stackrel{\sim}{~}}$ & 昌 & 三 & లై \\
\hline 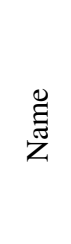 & 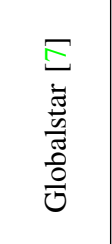 & 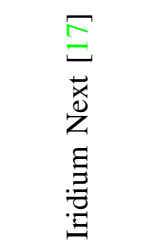 & 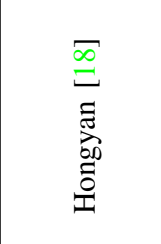 & $\begin{array}{l}\frac{\sigma}{2} \\
0 \\
00 \\
0 \\
0 \\
0\end{array}$ & 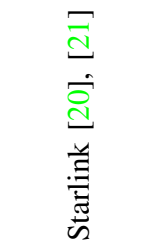 & 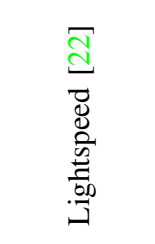 & $\begin{array}{l}\bar{d} \\
\frac{\bar{d}}{\overline{0}} \\
\frac{\vec{a}}{a}\end{array}$ & 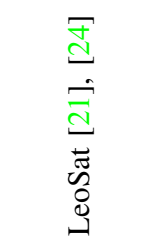 & 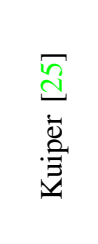 \\
\hline
\end{tabular}




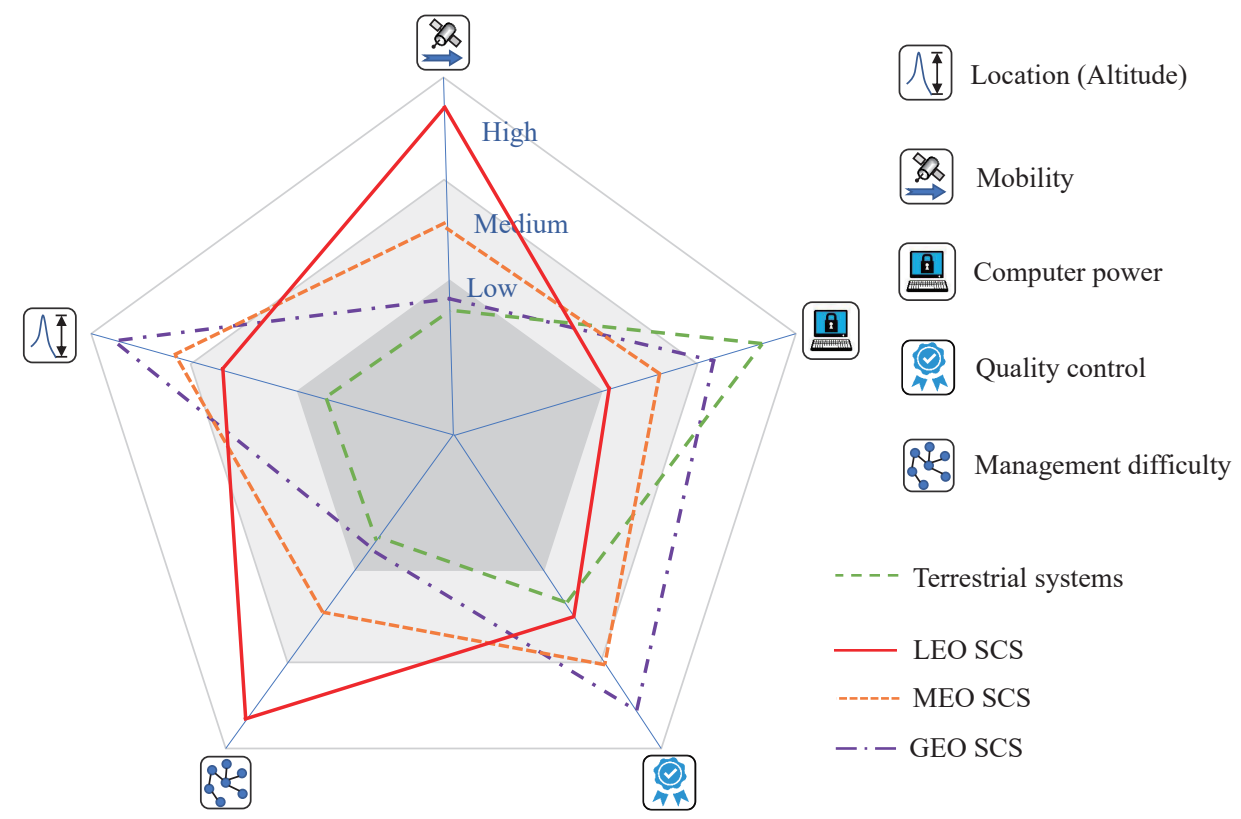

Fig. 2: The comparison of existing SCSs.

1) Eavesdropping: Lin et al. [38] proposed a robust secure Beamforming (BF) scheme for maximizing the achievable secrecy rate under the constraint of the total transmit power, which is a challenging non-convex optimization problem. A sequential convex approximation method was then employed for transforming it into a linear problem associated with a series of linear matrix inequalities and second-order cone constraints. Then the optimal BF weight vectors were obtained through an iterative algorithm. A low-complexity thresholdbased scheduling scheme was proposed by Guo et al. [39] for enhancing the secrecy performance of multiuser SCSs. Closed-form expressions were derived for the Secrecy Outage Probability (SOP) and Average Secrecy Capacity (ASC) of the system. Asymptotic expressions have also been obtained for the SOP and ASC to get deeper insights at high Signal to Noise Ratio (SNR). Xu et al. [40] discussed the secure transmission optimization of Intelligent Reflecting Surface (IRS)-assisted satellite-terrestrial integrated networks. Specifically, an IRS was employed for signal enhancement at the terrestrial network user, for interference suppression at the satellite user, and for degrading the reception at the eavesdropper.

2) Hijacking: There have been numerous contributions on dealing with the issues of hijacking [41]-[43]. Yi et al. [41] studied the vulnerability of intelligent early warning technology in the context of IoT networks. Vieira et al. [42] conceived an architecture for automated intrusion detection based on Big Data, with a special emphasis on the classification, understanding, and prediction of behavioral irregularities in distributed computing environments. He et al. [43] proposed a novel blockchain-based technique, which eliminated the security risks of a public key infrastructure. Hence it was robust against prefix hijacking attacks. A sophisticated amalgam of the credence value, collective signing, sharding, and of a penalty mechanism was conceived for safeguarding the consistency, scalability, and security of the system.
3) Node Compromise: A secure shortest path routing scheme relying on a reliable risk control algorithm was proposed by Wang et al. [44] for guarding against attacks by compromised nodes, which may be identified by deploying reputation-based systems. Chen et al. [45] considered the potential of blockchain-aided solutions designed for protecting the integrity of IoT networks. A stochastic blockchain-based data checking scheme was used for replacing the existing centralized approaches potentially leading to a single point of failure and network congestion. Pacheco et al. [46] employed artificial neural networks for intrusion detection in the context of IoT fog nodes. Their simulation results showed that this is a low-complexity scheme, imposing short execution time. Mohammad et al. [47] conceived a resilient password manager relying on physical unclonable functions, which harnessed redundancy in the proposed prototype, for avoiding password compromising events.

4) Interference: $\mathrm{Su}$ et al. [48] proposed a coverageexpansion method for LEO satellites supporting hybrid widespot beam coverage for avoiding any interference with GEO satellites. A heuristics-based Radio Resource Management (RRM) algorithm was designed for mitigating the interference between LEO and GEO satellites by Emiliano et al. [49], which was tested in a software simulator. A novel spectrum usage between GEO and LEO satellites was proposed by Wang et al. [50], which struck an attractive trade-off between the design complexity and the spectrum awareness accuracy. Ge et al. [51] proposed a Non-orthogonal Multiple Access (NOMA)-based GEO and LEO satellite network and employed Successive Interference Cancellation (SIC) for mitigating the interference.

5) Jamming: Liao et al. [52] designed a robust BF algorithm based on adaptive space-time processing for guarding against malicious jamming, which reduced the sidelobe level at a low complexity. A scheme based on classic Deep Reinforce- 


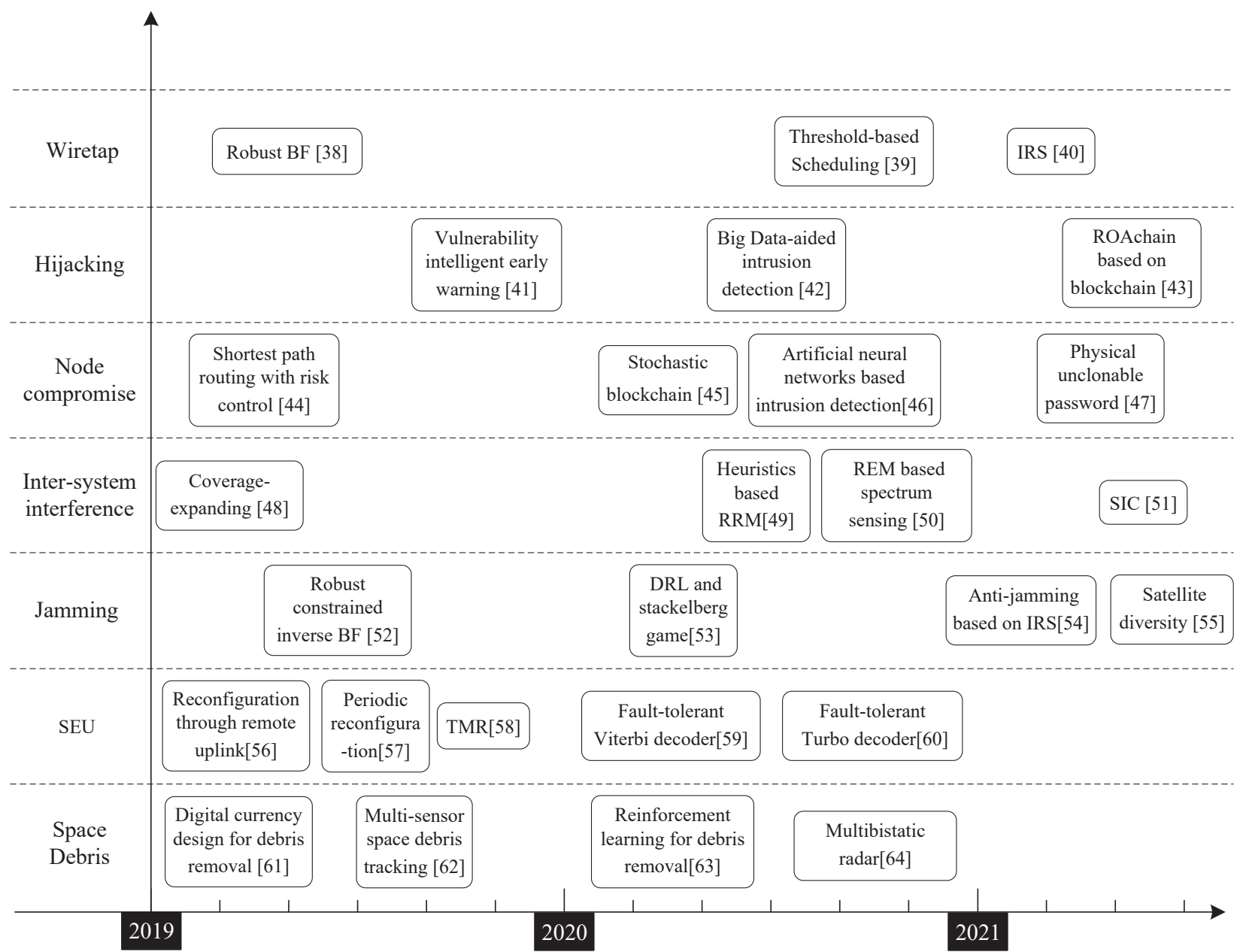

Fig. 3: Development timeline of related works for solving security vulnerabilities. The timing box indicates the major development period of the corresponding works, while the Y-axis indicates the security vulnerabilities.

ment Learning (DRL) and Stackelberg game-assisted spatial anti-jamming was proposed by Han et al. [53] for the Internet of Satellites. The communication countermeasures protecting satellite users against jammers were modeled as a Stackelberg anti-jamming routing game. Again, DRL techniques were used to deal with the interaction between the satellites and the jammers. Yang et al. [54] proposed a novel anti-jamming model based on IRSs. Jointly optimizing the power allocation and the IRS-based BF was formulated for enhancing the antijamming performance. Weerackody et al. [55] proposed the employment of satellite diversity for jamming mitigation in LEO mega-constellations.

6) Single Event Upsets: Rigo et al. [56] designed a radiation-hardened reconfigurable hardware platform, which has the ability to change the hardware configuration of the Field Programmable Gate Array (FPGA) employed by relying on the uplink control for mitigating the effect of SEUs. Monreal et al. [57] demonstrated that the periodic reconfiguration of Commercial Off The Shelf (COTS) components have the potential of mitigating the probability of SEUs. Gkiokas et al. [58] proposed a fault-tolerant processor for SEU mitigation by employing Triple Module Redundancy (TMR) voting applied to the read/write operation along with memory scrubbing. Gao et al. [59], [60] studied the fault tolerance of an SRAM-FPGAbased Viterbi decoder and Turbo decoder.

7) Space Debris: Saito et al. [61] viewed orbit debris as a kind of debt owed by the human race of existing generations to future generations. The debris removal 'currency' had the effect of transferring the corresponding credit to the present day. Hence the authors proposed to utilize a new digital currency associated with planned depreciation to build a sustainable economic model of debris removal. Wei et al. [62] proposed a multi-sensor space debris tracking scheme based on generalized labeled multi-Bernoulli filtering, which mitigated the detection uncertainty, data association uncertainty, and clutter in debris tracking. A reinforcement learning-based framework was conceived by Yang et al. [63] for solving the debris removal mission planning problem. Cataldo et al. [64] advocated the concept of multi-static radar for debris tracking. Its cost was low because already existing instruments such as illuminators and radio-telescopes were harnessed as receivers.

Against this backdrop, the objective of our paper is to provide a comprehensive survey of the security of LEO SCSs. Explicitly, the inherent characteristics, security vulnerabilities, security countermeasures, and the associated future perspectives are presented. 
In a nutshell, our contributions can be summarized as follows:

- We briefly review the evolution of LEO SCSs and their state-of-the-art, which is summarized in Table I at a glance. Furthermore, we discuss their inherent characteristics and outline their unique security challenges, as well as the deleterious effects of interference and that of space debris;

- Relying on recent research results we classify their security vulnerabilities into five categories, including passive and active eavesdropping attacks, as well as the impact of interference, SEUs, and space debris. Furthermore, the characteristics and impact of these security vulnerabilities are analyzed and summarized in Table IV at a glance;

- As a remedy, we review a rich suite of security countermeasures and classify them into active and passive security countermeasures, depending on whether they can proactively mitigate these security vulnerabilities.

- We illustrate the root causes of security vulnerabilities and the implementation of security countermeasures by carefully considering the trade-offs among numerous factors, such as the security, integrity, latency, complexity, etc. Bearing these trade-offs in mind, we infer tangible design guidelines;

- Finally, by analyzing the recent research results and the above-mentioned vulnerabilities, we highlight several promising future research directions, including secure quantum communications, three-dimensional (3D) virtual arrays, artificial intelligence-based security measures, space-based blockchain, and intelligent reflecting surface enabled secure transmission.

Indeed, there have been other security surveys and tutorials published in [65]-[72]. However, i) to the best of our knowledge, this is the first survey that provides a comprehensive security overview of LEO SCSs, and ii) our paper offers a cross-disciplinary synthesis ranging from information security to space-borne payload protection. Our contributions are boldly and explicitly contrasted to the other surveys in Table II for explicitly identifying the gaps in the literature.

\section{Paper Organization}

The organization of this paper is illustrated in Fig. 4. In Section II, the security vulnerabilities encountered by LEO SCSs are categorized. Section III introduces the family of active and passive security countermeasures conceived for safeguarding LEO SCSs. In Section IV, some open problems and research ideas concerning the security of LEO SCSs are provided. Finally, our concluding remarks and design guidelines for LEO SCSs are provided in Section V. The acronyms used in this paper can be found in the Table IX for convenience.

\section{Security Vulnerabilities}

LEO SCSs support more and more civilian and military applications, thus it is of paramount importance to eliminate their security threats. In this section, we focus our discussions on the security vulnerabilities of LEO SCSs shown in

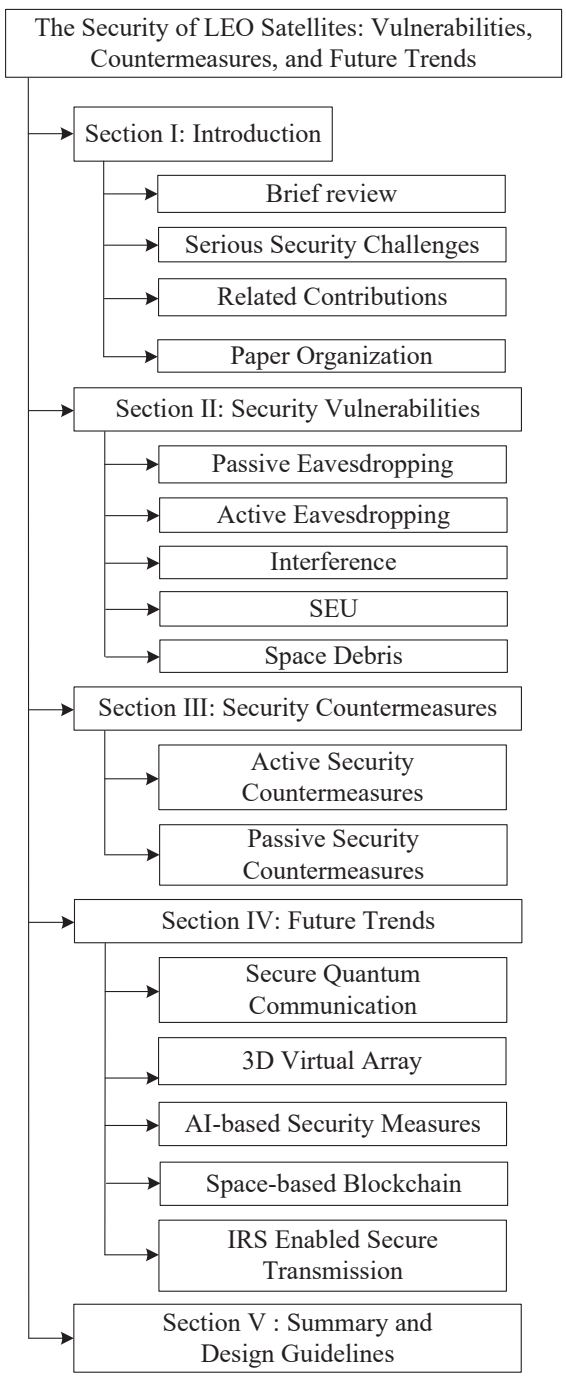

Fig. 4: Organization of this paper.

Fig. 5 at a glance, which may be divided into the following five categories: passive eavesdropping, active eavesdropping, interference, SEU, and space debris. The first three categories also have their subclasses. These vulnerabilities will be further detailed below.

\section{A. Passive Eavesdropping}

Passive eavesdropping aims for illegally obtaining confidential information during transmission. Hence their presence is hard to detect. Passive eavesdropping is divided into overhearing and transponder stealing, which will be introduced in more detail below.

1) Overhearing: Again, the open nature of wireless propagation makes legitimate transmissions vulnerable to eavesdropping, as seen in Fig. 6. The source transmits its signal to $\mathrm{D}$, while $\mathrm{E}$ is capable of overhearing the legitimate transmissions, if it is located in the coverage area of $\mathrm{S}$. This indicates a clear trade-off between the security and the integrity. Specifically, to improve the security of S, the conventional approach is to reduce the transmit power. However, the system's integrity will also be reduced simultaneously. 
TABLE II: Comparison with available surveys and tutorials

\begin{tabular}{|l|c|c|c|c|c|c|c|c|c|}
\hline \hline Paper & {$[65]$} & {$[66]$} & {$[67]$} & {$[68]$} & {$[69]$} & {$[70]$} & {$[71]$} & {$[72]$} & This work \\
\hline Year & 2015 & 2016 & 2018 & 2019 & 2020 & 2021 & 2021 & 2021 & 2021 \\
\hline Type & Tutorial & Survey & Survey & Survey & Tutorial & Survey & Survey & Survey & Survey \\
\hline Eavesdropping & $\checkmark$ & $\checkmark$ & $\checkmark$ & $\checkmark$ & $\checkmark$ & $\checkmark$ & & $\checkmark$ & $\checkmark$ \\
\hline Node compromise & $\checkmark$ & $\checkmark$ & $\checkmark$ & $\checkmark$ & & & & & $\checkmark$ \\
\hline Hijacking & & $\checkmark$ & & $\checkmark$ & & & & $\checkmark$ & $\checkmark$ \\
\hline Interference & & & & & $\checkmark$ & $\checkmark$ & & & $\checkmark$ \\
\hline Jamming & $\checkmark$ & $\checkmark$ & $\checkmark$ & $\checkmark$ & $\checkmark$ & $\checkmark$ & & $\checkmark$ & $\checkmark$ \\
\hline SEU & & & & & & & $\checkmark$ & & $\checkmark$ \\
\hline Transponder stealing & & & & & & & & & $\checkmark$ \\
\hline Space debris & & & & & & & & & $\checkmark$ \\
\hline Trade-offs & & & & & & & & & $\checkmark$ \\
\hline
\end{tabular}

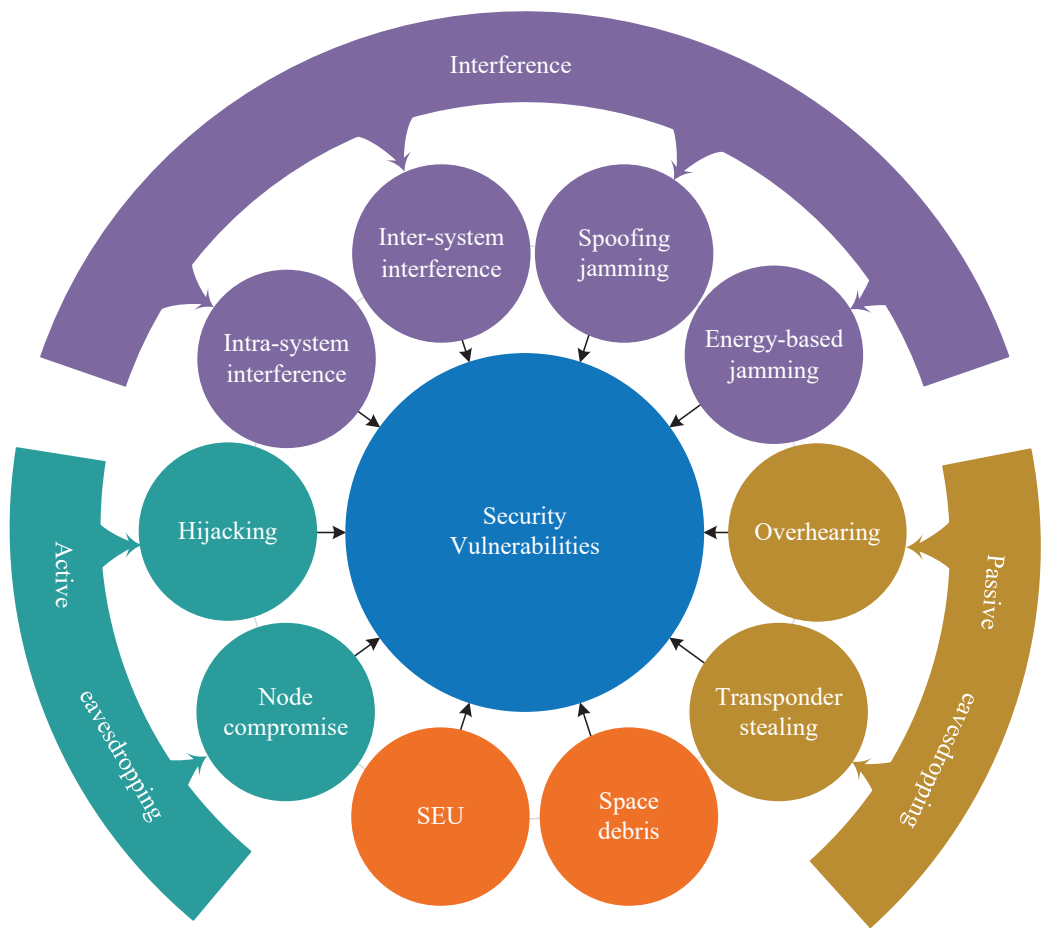

Fig. 5: Classification of security vulnerabilities.

Conversely, increasing the transmit power to improve the desired link's integrity, the probability of eavesdropping will also be inevitably increased [73].

Typically, cryptography techniques are adopted to prevent $\mathrm{E}$ from intercepting legitimate transmission between $\mathrm{S}$ and D. However, owing to the limited signal processing capability and power resources on-board of LEO satellites in the face of the potentially high computing power of the adversary, traditional cryptography is no longer a high-security solution. As a remedy, the family of information-theoretic security measures has been conceived, which exploits the random physical characteristics of wireless channels under the terminology of physical-layer security, which has been recommended for satellite-to-earth links, for example in [74]-[76].

2) Transponder Stealing: Transponder stealing constitutes another passive eavesdropping technique seen in Fig. 7, under which the eavesdropper adopts legitimate satellite transponders to transmit information. If the compromised satellites do not perform any signal processing, they are only harnessed for transparent forwarding [77]. Hence it is not possible to determine whether the received data is from a legitimate user. When attackers send their illegal signals, the satellite will still forward the signals [78]. For example, the $\mathrm{K} 3 \mathrm{H}$ transponder of the Asian No.7 satellite suffered unknown signal interference, some have suspected that hackers privately exploited the satellite transponder for their transmission [79].

Moreover, it is a challenge for legitimate users to detect, thus an eavesdropper, when it employs Direct Sequence Spread Spectrum (DSSS) techniques at a low Power Spectral Density (PSD) under the noise floor of the transponder's receiver. The authors of [80] proposed a sophisticated technique for tackling this problem. 


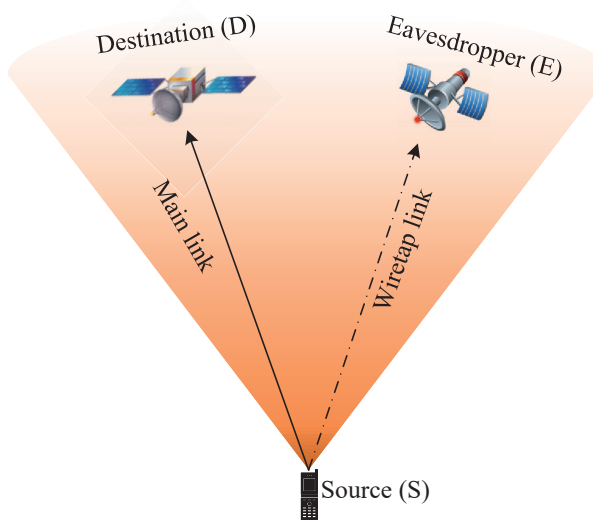

Fig. 6: A LEO SCS consisting of a source and a destination in the presence of an eavesdropper (uplink).

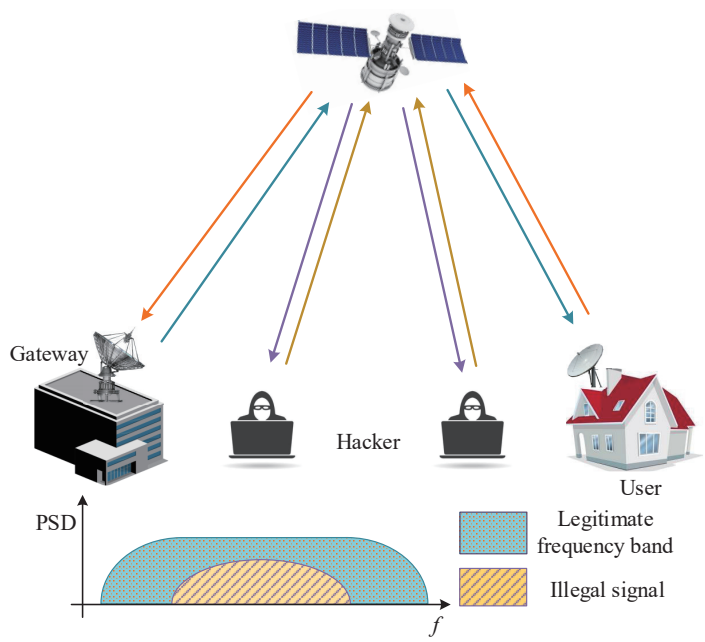

Fig. 7: Illustration of transponder stealing.

\section{B. Active Eavesdropping}

Active eavesdropping aims for disrupting the operation of legitimate systems by compromising or hijacking a node. This is typically achieved by imitating legitimate users for malicious access and communication [65]. Hijacking refers to the case when attackers hack into ground facilities in order to control satellites.

1) Node Compromise: In this scenario, a legitimate node is attacked by an adversary under the control of malicious algorithms, programs, or software, potentially threatening the entire network. Attackers may harness these compromised nodes for sending incessant requests, hence inducing network congestion [81]. More specifically, for satellite-IoT applications supported by LEO SCSs, the power-limited terminals operating without advanced security protection algorithms may suffer from a high risk of being captured by an adversary. The threat may be exacerbated by potential attacks from numerous geographically dispersed adversaries.

The IoT botnet in which malware source code was leaked in early 2015 is a typical paradigm of node compromise attacks [82]. IoT botnets, created as hackers, infect numerous IoT devices and recruit them to launch large-scale node compromise attacks. These attacks are difficult to detect and mitigate, because they use hit-and-run tactics that originate from numerous IoT vectors distributed around the world. Furthermore, these compromised nodes may also trick other legitimate nodes into compromised nodes [83]. It is challenging to detect compromised nodes, because the behaviour of these compromised nodes and legitimate nodes is hard to distinguish. Using code patches is a common method of mitigating the probabilities of these events [65].

2) Hijacking: LEO SCSs provide a powerful platform for military applications, which are hence prime targets for hostile attacks. Their facilities on the ground are responsible for all interactions with other terrestrial networks, and these facilities create opportunities for hackers [28]. Additionally, low-cost COTS components may also open the door for hackers. Some satellite manufacturers, especially CubeSat, employ off-theshelf technology to reduce costs, and the widespread availability of these low-cost COTS components also reduces the costs for hackers.

Furthermore, hackers can turn off satellites in batches by taking over the telemetry, tracking, and control link of LEO satellites, hence causing global service interruptions. They may also block or spoof signals from satellites, thereby causing damage to critical infrastructures, such as power grids, water supply networks, and transportation systems connected to the Internet of satellites. Hackers could control satellites to achieve self-destruction by malicious commands, or they can use special tools to trick satellites and ultimately use them to attack other satellites or space assets. Fortunately, there are potent security countermeasures against hijacking attacks [84].

\section{Interference}

Interference can be roughly divided into two types: unintentional interference and intentional interference, both of which degrade the quality of legitimate communications. To elaborate, unintentional interference is caused by the deficient design of legitimate systems or inappropriate frequency management among systems, hence inflicting both intra-system interference and inter-system interference. Intentional interference known as jamming represents the radio signal transmitted by the adversary, which falls into two categories: spoofing jamming and energy-based jamming. Next, we will introduce these four types of interference sources in more detail.

1) Intra-system Interference: Spread Spectrum (SS) techniques are eminently suitable for LEO SCSs in military applications, which are immune to most types of interference to a certain extent. However, it is difficult to avoid the nearfar effect caused by Multiple Access Interference (MAI) [85], where a CCI degrades the designed signal. Power control and multi-user detection are common methods of mitigating these near-far effects [7]. Additionally, the careful choice of SS codes may mitigate the near-far effects. Orthogonal complementary codes have been chosen to substantially mitigate MAI [86]-[88]. However, these orthogonal codes are sensitive to frequency shifts, which must be mitigated by future research.

Multi-beam satellites reuse the available frequencies within their coverage to increase capacity. However, frequency reuse 
among beams may cause CCI in the overlapping areas, when some beams rely on the same frequency [89], especially in adjacent beams using the same frequency. The angular sidelobes of the beam radiation patterns create interference leakage seen in Fig. 8. The interference level is typically quantified in terms of the Carrier to Interference Ratio (CIR). Clearly, the interference limits the attainable capacity. To improve the capacity, the conventional approach is to increase the distance between the positions using the same frequency. As a remedy, Transmit Precoding (TPC) techniques relying on transmitter side channel state information can be applied for mitigating the interference. A potent scheme based on hybrid wide-spot beams was designed for alleviating this source of interference in [48]. The main philosophy of this scheme is that the space-borne payload generates several fixed wide beams for providing wide-range coverage, so as to increase the frequency reuse distance. On this basis, the space-borne payload also adopts some high-gain spot beams for enhancing the capacity in tele-traffic hot spots.

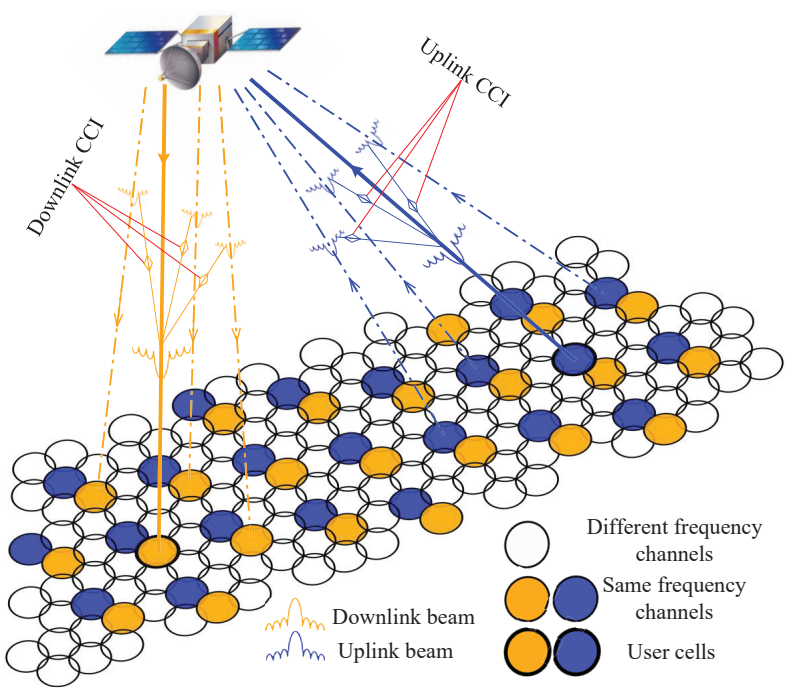

Fig. 8: Depiction of the satellite uplink and downlink CCI.

2) Inter-system Interference: An increasing number of LEO satellites has been deployed over the last few years, but the available radio spectrum remains limited. So LEO satellites require high spectral efficiency to address the spectrum scarcity problem. Furthermore, GEO SCSs have to coexist within the same spectrum in order to achieve this objective. Consequently, having high inter-system interference between LEO and GEO SCSs is unavoidable. When LEO satellites [90] approach the equator, they tend to inflict increased interference upon GEO satellites operating within the same frequency band, as shown in Fig. 9. According to current International Telecommunications Union (ITU) regulations, it is mandating to consider the spectrum sharing between GEO and LEO SCSs. LEO SCSs shall not impose unacceptable interference on GEO SCSs. In other words, GEO SCSs are regarded as the Primary User (PU), while LEO SCSs are regarded as the Secondary User (SU). Thus interference coordination is imperative for mitigating the interference.

On the other hand, the next-generation networks will pro-

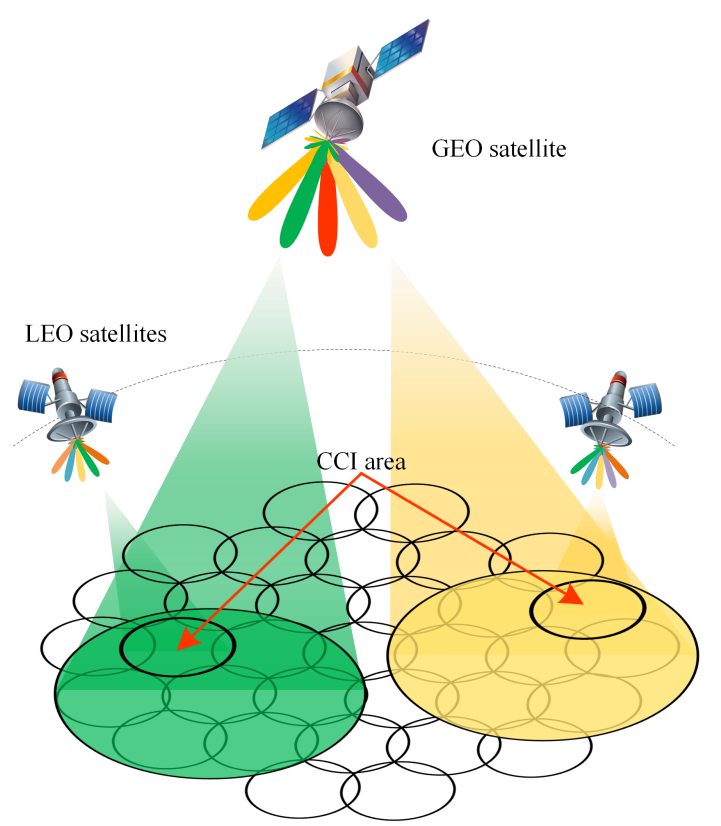

Fig. 9: Inter-system interference between LEO and GEO SCSs.

vide ubiquitous connectivity through the convergence of terrestrial systems, LEO SCSs, and GEO SCSs [91]. However, the coexistence with LEO and GEO SCSs has to be carefully planned. Adding terrestrial systems to the mix makes an already complicated picture more complex.

3) Spoofing Jamming: Spoofing jamming is a form of electronic attack where the attacker tricks a receiver into believing in the genuine nature of a malicious signal produced by the attacker. For example, spoofing jamming often occurs in the civilian Global Position System (GPS). It is easy for the adversary to release spoof GPS signals to provide false information, because the format of the civilian GPS signal is known [92]. Similar to GPS, there is usually a dedicated downlink pilot channel for broadcasting channel status, user management information, call information, etc. as exemplified by Iridium [6]. Attackers can imitate the dedicated pilot channel to broadcast false information to legitimate users, causing network paralysis. Fortunately, there are some common methods to alleviate spoofing jamming, such as energy detection, multiple antennas [93], and authentication. However, energy detection and multiple antennas increase the terminal complexity. Hence the most effective approach is to apply authentication for LEO SCSs. The authors of [94] proposed an Unmanned Aerial Vehicle (UAV) [95], [96]assisted authentication method to tackle spoofing jamming.

4) Energy-based Jamming: Energy-based jamming is also a form of electronic attack that interferes with Radio Frequency (RF) communications by generating noise in the same frequency band and within the field of view of the antenna at the targeted receiver. For example, most of the on-orbit satellites adopt the so-called bent-pipe ${ }^{1}$ transponder without

\footnotetext{
${ }^{1}$ Many satellites send back to Earth what goes into the satellite with only amplification and a shift from uplink to downlink frequency, like a bent pipe. A bent-pipe satellite does not demodulate and decode the signal.
} 
TABLE III: Comparison of energy-based jamming techniques

\begin{tabular}{|c|c|c|c|}
\hline \hline $\begin{array}{c}\text { Jamming } \\
\text { types }\end{array}$ & $\begin{array}{c}\text { Space-based } \\
\text { jamming }\end{array}$ & $\begin{array}{c}\text { Air-based } \\
\text { jamming }\end{array}$ & $\begin{array}{c}\text { Ground-based } \\
\text { jamming }\end{array}$ \\
\hline $\begin{array}{c}\text { Jamming } \\
\text { power }\end{array}$ & Low & Medium & High \\
\hline $\begin{array}{c}\text { Jamming } \\
\text { time }\end{array}$ & Burst & Burst & Continuous \\
\hline Resources & Limited & Limited & Rich \\
\hline Mobility & Poor & Strong & Poor \\
\hline $\begin{array}{c}\text { Sphere of } \\
\text { action }\end{array}$ & Large & Medium & Small \\
\hline Scenarios & downlink & $\begin{array}{c}\text { downlink } \\
\text { uplink }\end{array}$ & uplink \\
\hline
\end{tabular}

digital signal processing, so it is easy to encounter signal energy-based jamming attack. Attackers may easily perturb the satellite's operation by transmitting high-power jamming signals [97]. There are many types of jamming signals and classification methods. Zou et al. [66] classified jamming based on the grade of difficulty generating them and compared the different types of jamming schemes in terms of their energy efficiency, how disruptive their interference is their complexity, and the prior knowledge.

Due to the long open wireless link between LEO satellites and the Earth, the adversary may contaminate it by jamming at different locations, which can be divided into the types illustrated in Fig. 10.

Space-based Jamming: The space-based jamming is mainly released by spacecraft. This type of jamming has a large range over which it may disrupt the downlink transmission, but it has limited jamming time and power owing to having limited time above the horizon.

Air-based Jamming: The adversary may generate air-based electronic jamming from aircraft or airships. As the electronicjamming aircraft and airships have more flexibility than their space-based counterparts, they are suitable for releasing bursttype jamming. Compared to space-based jamming, the power of air-based jamming is typically higher. Because airships are generally located between the ground users and LEO satellites, they can interfere with the desired communication during both uplink and downlink transmissions.

Ground-based Jamming: The power of ground-based jamming is typically high and the jamming style is diverse, because the ground-based jamming is maliciously released by large-scale fixed, vehicle-mounted, or ship-borne jamming stations having abundant resources and power. Ground-based jamming mainly affects the uplink transmissions. There are many types of ground-based jamming, but the distance is not a dominant factor. Ground-based jamming is usually of blocking nature, which directly blocks the satellite transponder. These three types of energy-based jamming techniques are compared in Table III.

The family of SS modulations constitutes efficient techniques resisting jamming. Additionally, some non-SS antijamming techniques including, temporal domain adaptive filtering [98] and transform domain adaptive filtering [99], can be employed for mitigating jamming.

\section{Single Event Upsets}

The particles existing in cosmic radiation generate a large number of electrons and holes in the incident path by ionization. Electronic devices like FPGAs collect these charges, which may cause transient faults. If the charge exceeds the maximum level that the device can withstand without SEU, the logic state of the circuit will be inverted. However, the circuit can be restored to its original working state by rewriting or resetting. Hence SEUs constitute reversible soft errors [100].

The nature of SEUs is hardware-dependent. Compared to FPGAs, Application Specific Integrated Circuits (ASICs) exhibit better resistance to SEU, but they lack flexibility. Therefore, FPGAs are widely used in LEO satellites as a benefit of their high performance and flexibility. In order to ensure the reliable operation of FPGA in-orbit, it is necessary to employ SEU mitigation measures, such as TMRs and periodical refreshing.

\section{E. Space Debris}

In recent years, the launch activities have been increasing for LEO, Medium Earth Orbit (MEO), GEO satellites. The different orbital regions are unevenly populated. It is seen from Fig. 11 that the LEO orbits between 800 and $1400 \mathrm{~km}$ constitute the most crowded space fuelled by the miniaturization of satellites and the deployment of mega-constellations.

However, frequent launch activities in LEOs greatly increases the risk of collisions, which inevitably generate further debris. As a matter of fact, in 2009, the Iridium 33 satellite collided with the scrapped Russian Cosmos over Siberia, producing at least thousands of debris [102]. These space debris was fixed only a few months later, distributed between $500 \mathrm{~km}$ and $1300 \mathrm{~km}$. As a remedy, the collision avoidance control has to be carried out for reducing the risk of collisions with LEO satellites. On Sep. 2, 2019, European Space Agency (ESA) made an emergency steering of the Aeolus satellite, successfully avoiding a space 'car accident' with Starlink-44 [103]. As reported by United Nation Office for Outer Space Affairs, the China Space Station has successfully conducted two evasive manoeuvres to avoid potential collisions with the Starlink-1095 satellite on Jul. 1, 2021 and the Starlink-2305 satellite on Oct. 21, 2021, respectively [104].

Again, such frequent deployment activities have also led to a surge in space debris. Most orbital debris is humangenerated objects, such as pieces of spacecraft, tiny flecks of paint from a spacecraft, parts of rockets, and decayed satellites. According to the ESA, there are approximately 1036500 debris objects larger than $1 \mathrm{~cm}$ estimated by statistical models to be in orbit [105]. There are close to 6000 tons of materials in LEO. Most 'space debris' moves fast, reaching speeds of 18000 miles per hour, almost seven times that of bullets. They expose LEO satellites to the Kessler phenomenon ${ }^{2}$.

\footnotetext{
${ }^{2}$ The Kessler phenomenon, proposed by National Aeronautics and Space Administration (NASA) scientist Donald J. Kessler in 1978, is a chain reaction in which the resulting space debris would destroy other satellites and so on, with the result that LEO would become unusable [106].
} 


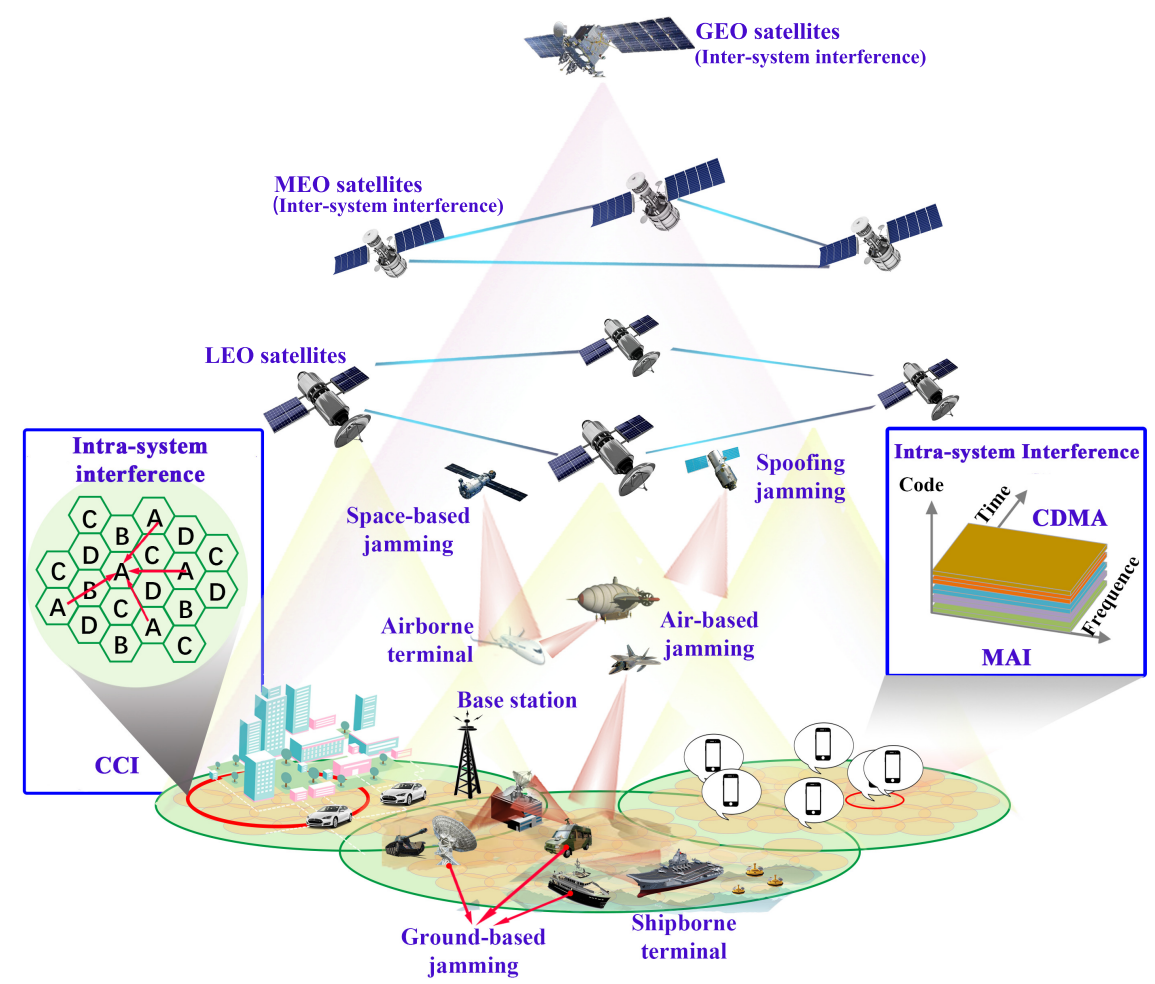

Fig. 10: Sources of interference contaminating LEO SCSs.

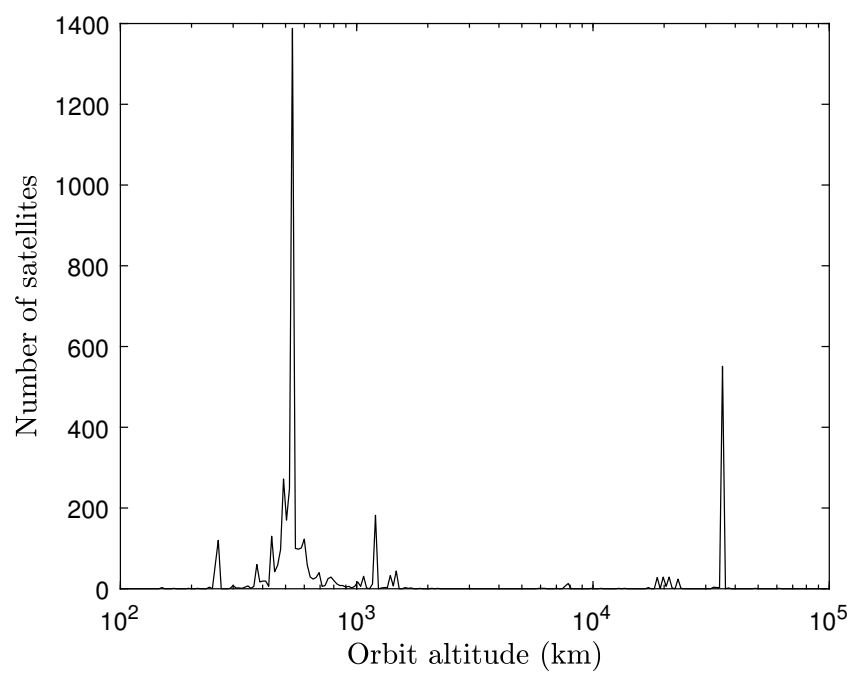

Fig. 11: Launch of satellites in different orbits during Sep. 2010 and Sep. 2021 [101].

Specifically, the density of space debris in LEO is high enough to cause cascade collisions, which adversely affects space exploration. With the advent of standardized production, the satellite development cycle and constellation deployment cycle have been substantially shortened, but there are also satellite failures, potentially requiring replacements during the deployment. Hence Kessler's hypothesis is becoming a reality.

As a matter of fact, collisions with debris at LEO orbits have already occurred [107]-[110], as shown in Fig. 12. Explicitly,
ESA has showcased the solar cells retrieved from the Hubble Space Telescope, which have been damaged by various collisions with space debris. In 2007, orbital debris completely penetrated one of the radiator panels of the shuttle Endeavour. On August 23rd, 2015, ESA engineers have discovered that a solar panel on the Sentinel-1A satellite was hit by a millimetersized debris according to space-borne cameras. Fortunately, this satellite still remained capable of operating normally. Fig. 12(d) shows the location of a space debris strike on the International Space Station's Canadarm2 robot arm, which was spotted on May 12th, 2021.

Because of these incidents, it is routine for operators of satellites in dense orbits to spend time on tracking the collision risk. When the probability of collision exceeds a certain limit, debris avoidance maneuvers have to be planned. For example, the International Space Station has carried out as many as 29 debris avoidance maneuvers since 1999 [111]. However, due to its excessive fuel consumption, the technical solutions in [111] are not suitable for low-cost LEO satellites with limited energy. Indeed, active debris removal is the best method of reducing the probability of collision, which will be described in detail in Section III.

In Table IV, we summarize, classify, and compare the security vulnerabilities encountered by LEO SCSs in terms of their types, attack location, degree of damage, reversibility, awareness, and collateral damage.

\section{Security Countermeasures}

In this section, a series of security countermeasures are presented as solutions for the aforementioned security vul- 


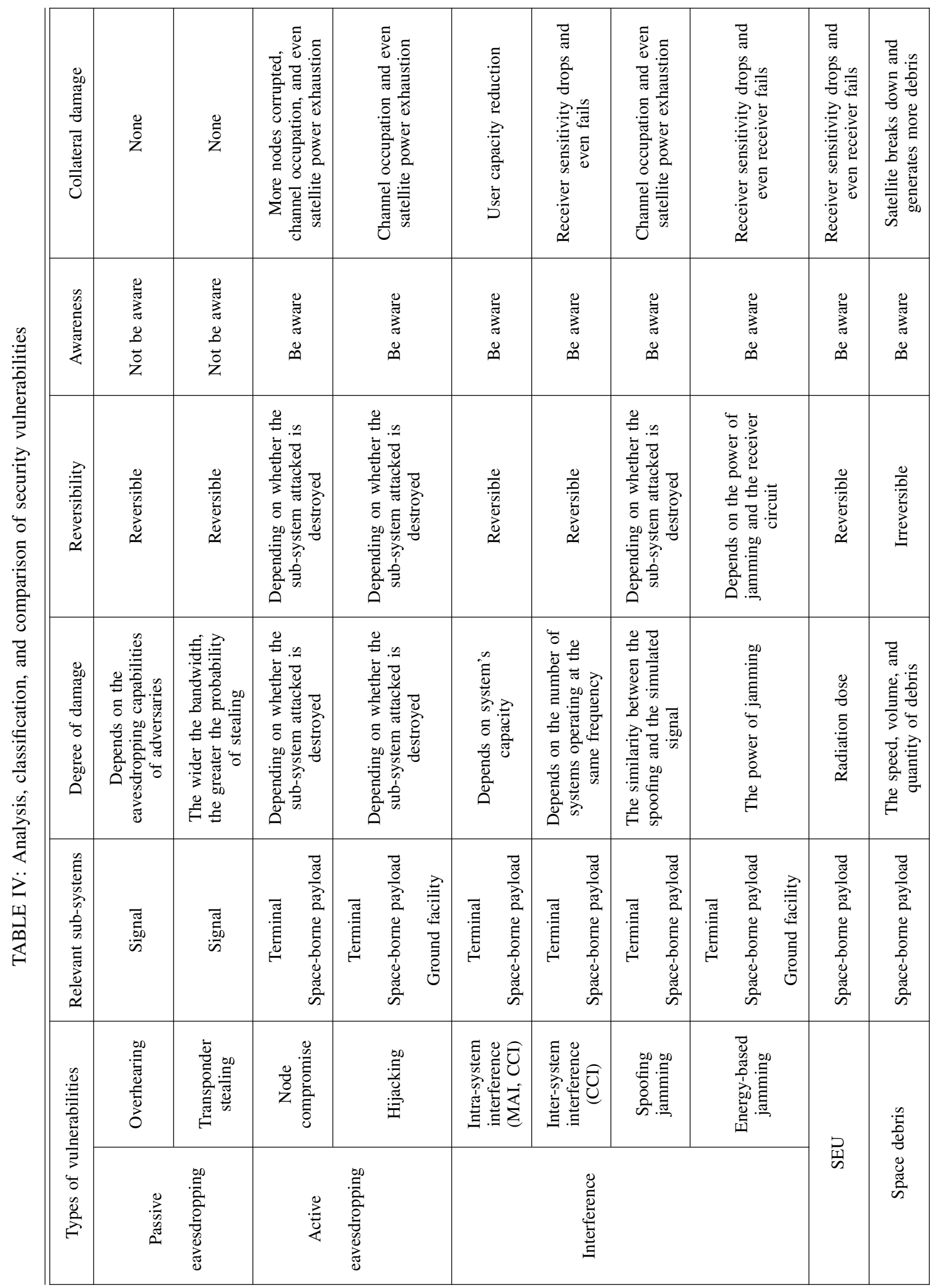




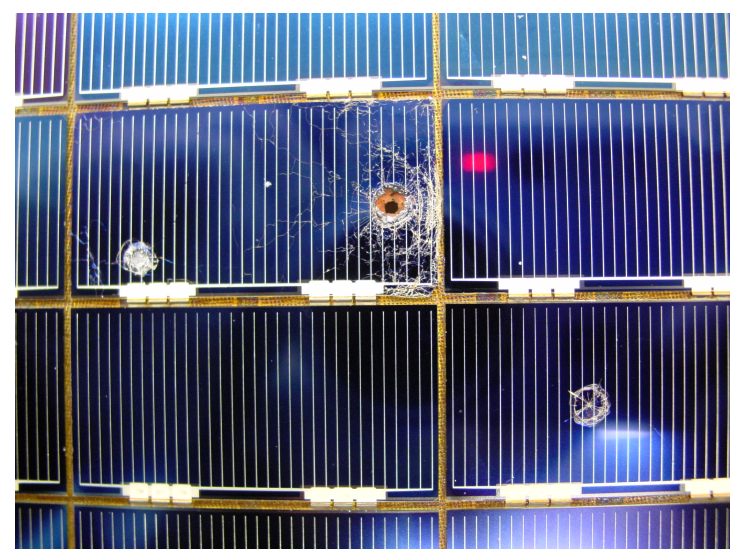

(a) Solar cells retrieved from the Hubble Space Telescope (b) (Credit: ESA.) [107].
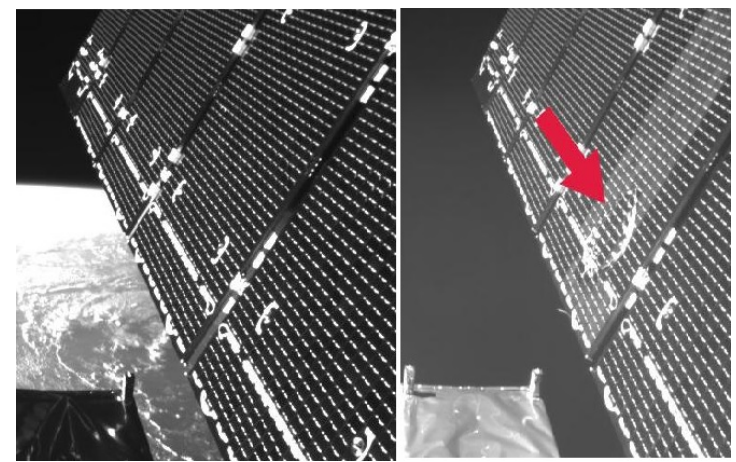

(c) Shot of the solar array struck by space debris on Sentinel 1-A satellite (Credit: ESA.) [108].

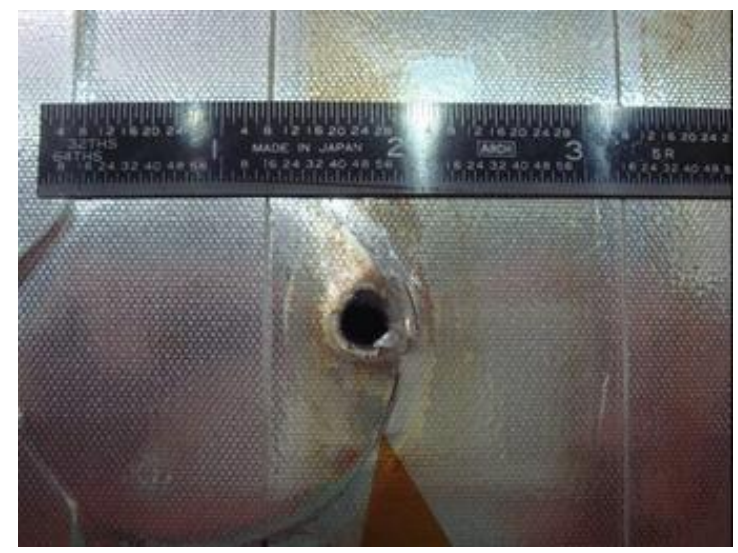
[108]

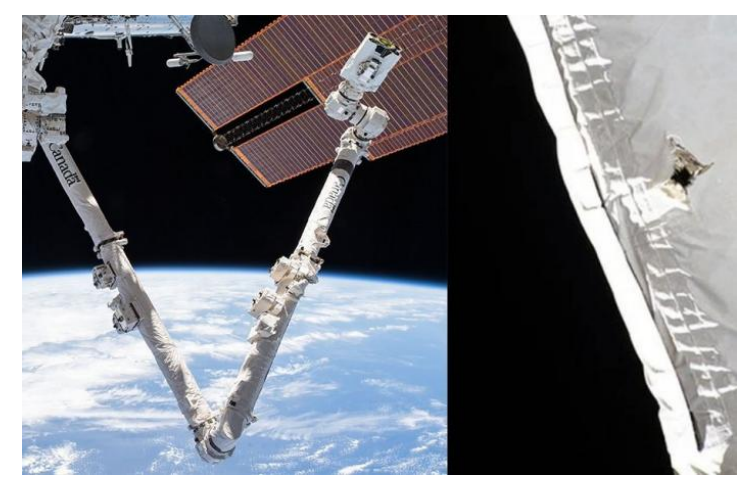

(d) Space debris hit a robotic arm on the International Space Station (Credit: NASA.) [110].

Fig. 12: Four examples of collisions with space debris in LEO orbits.

nerabilities. These countermeasures are mainly divided into active and passive security countermeasures. By definition, active security countermeasures are capable of avoiding, hindering, and even suppressing eavesdropping and interference. By contrast, passive security countermeasures must directly face these security threats and reduce or even eliminate the impact of eavesdropping and interference. Additionally, some security countermeasures such as debris removal and SEU mitigation measures aim for solving the problem of excessive space debris and SEU, respectively. The relationships between security vulnerabilities and countermeasures are demonstrated in Fig. 13.

\section{A. Active Security Countermeasures}

Advanced security countermeasures proactively aim for avoiding security vulnerabilities. Among them, advanced security-oriented antennas strive for enhancing the performance of legitimate users (desired signals), while mitigating the deleterious effects of both eavesdropping and interference. Interference cooperation is capable of preventatively tilting the beam in LEO SCSs before the occurrence of CCI between GEO and LEO SCSs. Additionally, space debris removal is capable of cleaning up space debris, thereby reducing the probability of collision. These active security countermeasures will be further detailed below.
1) Advanced Security-Oriented Antennas: There are recent studies on advanced security-oriented antennas for secure transmissions, since they are capable of reinforcing the radiation pattern in the direction of the desired receiver while suppressing the pattern in most of the other directions. However, an eavesdropper equipped with a sensitive receiver may still be capable of intercepting the communication link via a sidelobe. To tackle this problem, side-lobe randomization [112] may be used for alleviating side-lobe information leakage. The advanced security-oriented antennas employ BF and Artificial Noise (AN) [113] in the downlink to transmit AN in the direction of eavesdroppers for actively suppressing eavesdropping [114], [115].

However, as shown in Fig. 14, eavesdroppers may be able to penetrate the main-lobe direction anywhere between the satellite and the Earth. In this scenario, the aforementioned secure techniques no longer work, as their beams are only angle-dependent. The Frequency Diverse Array (FDA) [116] can be employed to address this problem. The authors of [117] introduced a Linear Frequency Diverse Array (LFDA) that can generate a beam pattern depending on both the angle and the distance by linearly shifting the carrier frequencies across different antennas. However, the distance and direction of the beam pattern generated are coupled, hence it may still be possible for the eavesdropper to incept the message of 


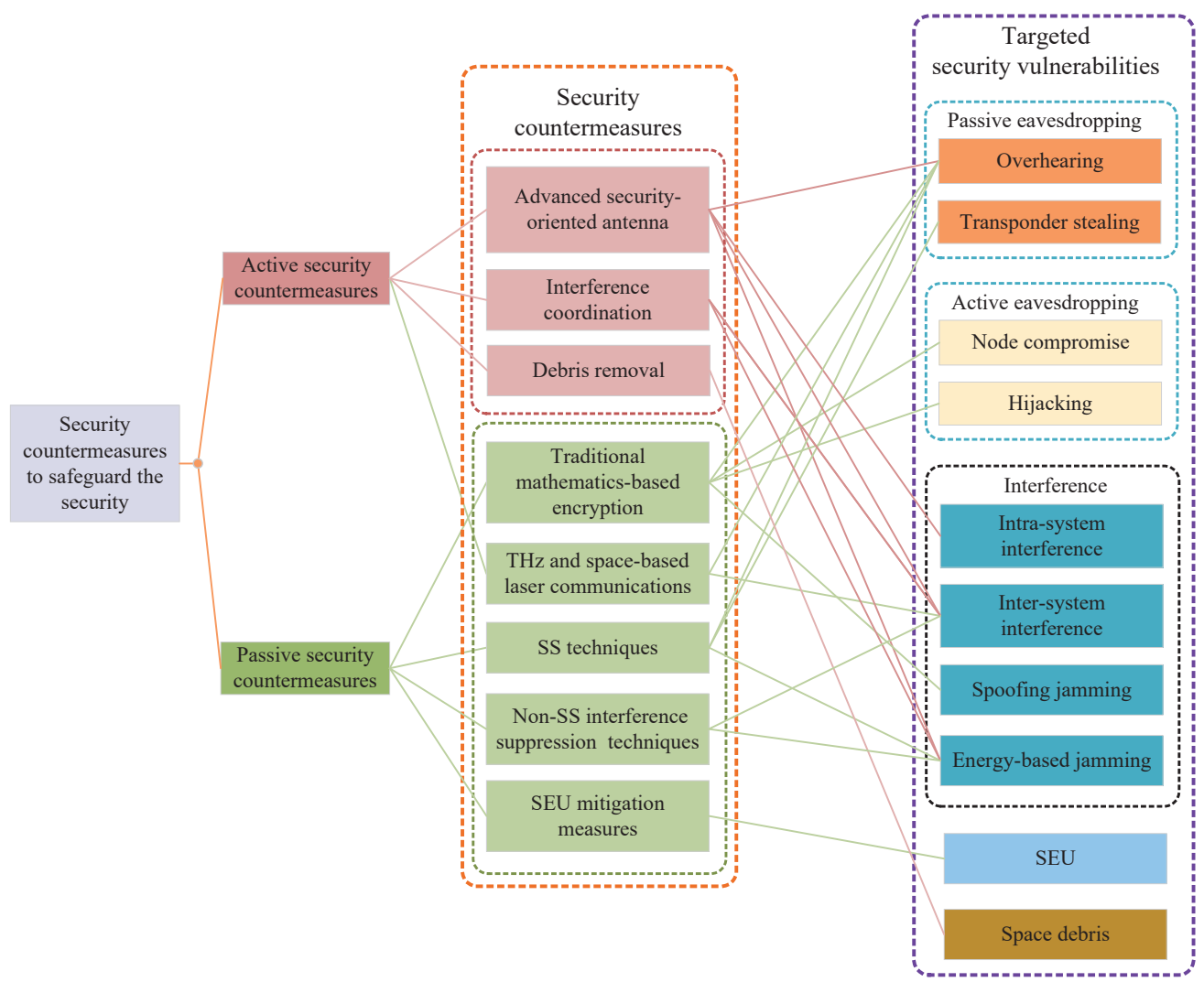

Fig. 13: Security countermeasures and their targeted security vulnerabilities.

the legitimate user at certain positions. Recently, the Radom Frequency Diverse Array (RFDA) concept was conceived [118], whose array elements were randomly assigned different frequencies for decoupling the correlation between direction and distance. Nevertheless, there are several drawbacks of the RFDA. Due to the randomness in the frequency of each array element, the implementation complexity of the terminal is increased. Although a FDA is capable of providing additional security in the distance dimension, its beam pattern is timevariant, which limits its field of application [119].

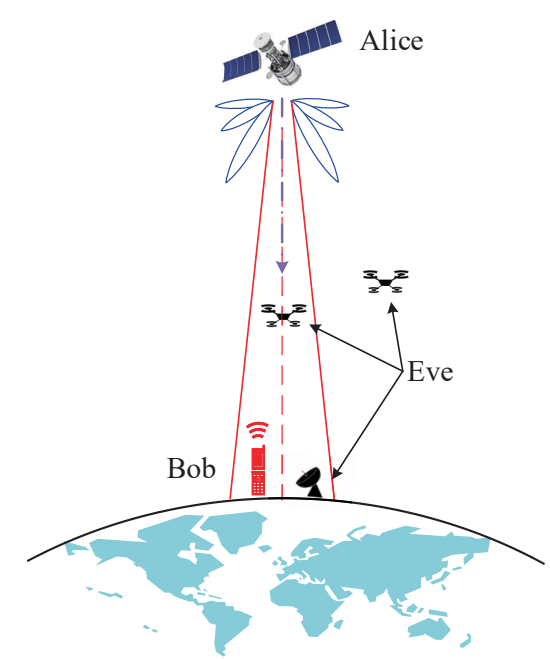

Fig. 14: Eve is aligned with Bob in the main-lobe's direction.
Relay cooperation constitutes a potential solution to the above problems [120], [121]. Each relay could send its downlink traffic information in the desired direction and $\mathrm{AN}$ in other directions to meddle with the eavesdropper's signal reception [122]. Additionally, relay cooperation also supports secure non-line-of-sight transmissions. However, the topology changes frequently due to the high mobility of LEO SCSs. Hence both the location and power of each node should be optimized for maximizing security performance.

Additionally, the NOMA-based TPC scheme is capable of improving the security, regardless of the specific location of E. Explicitly, recall from Fig. 6 that $\mathrm{S}$ can deliver both the legitimate signal as well as the interference used for confusing E, because similar to the concept of power-domain NOMA, the specifically conditioned interference may be superimposed on the legitimate signal. Successive interference cancellation (SIC) may be invoked by D for first detecting the higher-power specifically conditioned interference designed for confusing $\mathrm{E}$, which then leaves the clean desired signal behind.

However, there is a trade-off between the power assigned to the specifically conditioned interference designed for confusing $\mathrm{E}$ and the secrecy capacity improvement attainted. The maximum transmit power at $\mathrm{S}$ is written as $P$, while $P_{s}$ given by $0<P_{s}<P$ indicates the transmit power of the legitimate signal. Both the small-scale fading and the path loss are incorporated into the ordered channel gain. Based on the aforementioned assumptions, the instantaneous SNR of E and $\mathrm{D}$ can be written as 


$$
\begin{gathered}
\gamma_{S E}=\frac{\frac{P_{S}\left|h_{S D}\right|^{2}}{d_{S D}^{n}}}{\frac{P_{E}\left|h_{S E}\right|^{2}}{d_{S E}^{n}}+N_{0}} \\
\gamma_{S D}=\frac{P_{S}\left|h_{S D}\right|^{2}}{d_{S D}^{n_{1}} N_{0}}
\end{gathered}
$$

respectively. The channel's power gain over the satelliteterrestrial link, namely, $h_{S D}^{2}$, can be modeled as

$$
f_{\left|h_{S g}\right|^{2}}(x)=\alpha_{S g} \exp \left(-\beta_{S g} x\right){ }_{1} F_{1}\left(m_{S g} ; 1 ; \delta_{S g} x\right), x \geq 0,
$$

Hence, the ergodic secrecy capacity is given by

$$
\bar{C}_{S}=\mathbb{E}\left[\left(\log _{2}\left(1+\gamma_{S D}\right)-\log _{2}\left(1+\gamma_{S E}\right)\right]\right.
$$

where $\alpha_{S g}=\left(\frac{2 b_{S g} m_{S g}}{2 b_{S g} m_{S g}+\Omega_{S g}}\right)^{m_{S g}} /\left(2 b_{S g}\right), \beta_{S g}=\frac{1}{2 b_{S g}}$, $\delta_{S g}=\frac{\Omega_{S g}}{2 b_{S g}\left(2 b_{S g} m_{S g}+\Omega_{S g}\right)}, \Omega_{S g}$ and $2 b_{S g}$ are the average power of the LoS and multi-path components, respectively, $m_{S g}$ is the fading severity parameter, and ${ }_{1} F_{1}(\cdot ; 1 ; \cdot)$ is the confluent hyper-geometric function of the first kind.

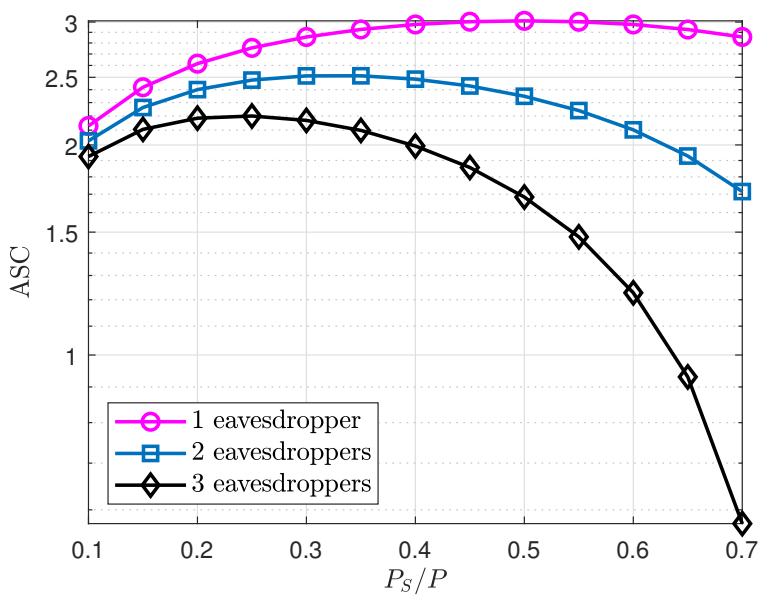

Fig. 15: The relationship between the ASC and $\frac{P_{s}}{P}$. The simulation parameters are as follows. The fading factor $m$ and $\Omega$ are given by 2 and 10 , respectively. The distance is $200 \mathrm{~km}$ and the $P$ is $20 \mathrm{dBW}$.

Aggressive frequency reuse results in high probability of CCI. The family of advanced security-oriented antennas [123] strikes a trade-off between the maximization of the signal power at the desired receiver and the minimization of the interference leaked to non-intended receivers. For this reason, sophisticated TPC techniques relying on advanced securityoriented antennas are required for mitigating the interference in order to facilitate adaptive coverage provision and dynamic traffic optimization [124]. The authors of [125] focused their attention on massive Multiple Input Multiple Output (MIMO) transmission for LEO SCSs by relying on sophisticated Doppler and delay compensations at the terminal.
In this way, the maximization of both the Average Signal to Leakage Plus Noise Ratio (ASLNR) and of the Average Signal to Interference Plus Noise Ratio (ASINR) was achieved.

The authors of [126] conceived a NOMA-based multi-beam LEO satellite-based IoT solution for mitigating the MAI of traditional orthogonal multiple access schemes. A robust BF design was formulated for minimizing the total transmit power of both non-critical and critical IoT applications.

The advanced security-oriented antennas also allow the beam pattern to be adjusted in response to time-variant jamming conditions. Explicitly, the beam pattern can be adjusted in azimuth to minimize the interference impinging from the left or right of an antenna or in elevation [127]-[129]. However, angular-domain anti-jamming schemes fail to suppress the jamming, when the legitimate user and the jamming are aligned in the same direction. The combination of temporal domain, transform domain, and spatial domain suppression [130], [131] represents a low-complexity solution in the case when the legitimate user and interference are aligned in the same direction. If there is still some residual interference in the signal after spatial domain jamming suppression, either temporal domain or transform domain adaptive filtering can be employed for further reducing the interference. The salient research advances in the field of advanced security-oriented antennas are summarized in Table $\mathrm{V}$.

2) Interference Coordination: Interference coordination is a promising technique of mitigating the inter-system interference caused by the spectrum crunch in SAGINs. It typically mitigates the interference by power control, beam drifting, cognitive radio techniques, etc. while improving the spectral efficiency and meeting the ever-increasing capacity demands.

The ITU specifies that GEO SCSs have priority over LEO SCSs with regard to frequency usage. Consequently, accurate power control is required in LEO SCSs for satisfying the interference constraints imposed by GEO SCSs. However, the power control also directly affects the throughput of LEO SCSs [132], [133]. As a remedy, the authors of [134] modeled this power control problem as an optimization problem aiming for maximizing the sum rate of the LEO SCSs. Then, the popular fractional programming technique was employed for transforming this nonconvex problem into a tractable form. By contrast, the authors of [135] conceived a joint multibeam power control algorithm for optimizing the transmit power of LEO and GEO satellite beams. On the premise of ensuring the signal quality of GEO SCSs. This algorithm judiciously reduced the transmission power of GEO beams, thereby maximizing the throughput of LEO SCSs.

Some schemes rely on so-called beam drifting in LEO SCSs, which force the LEO satellite users into the adjacent beam even before interference actually occurs [48], [136][138]. The authors of [136] conceived a sophisticated strategy for reducing the downlink interference inflicted by LEO satellites on GEO satellite users. The authors of [137] mitigated the interference between LEO and GEO satellites by appropriately tilting the transmission direction of the phased array based antennas of LEO satellites by solving a nonlinear programming problem used for finding the optimal direction. OneWeb adopted the method of [138] for LEO SCSs to avoid 
TABLE V: The evolution of advanced security-oriented antennas

\begin{tabular}{|c|c|c|c|}
\hline Target problems & Author(s) & Contribution & Year \\
\hline Overhearing & S. Goel et al. [112] & $\begin{array}{l}\text { Ensures secure communication by transmitting AN to } \\
\text { directions other than legitimate users. }\end{array}$ & 2008 \\
\hline Energy-based jamming & P. Rocca et al. [127] & $\begin{array}{l}\text { The dynamical configuration of the linear thin array is } \\
\text { proposed to form lower level side-lobes and nulls. }\end{array}$ & 2011 \\
\hline $\begin{array}{l}\text { The eavesdropper and legitimate } \\
\text { user are in the same direction }\end{array}$ & W. Wang [117] & $\begin{array}{r}\text { Proposes LFDA forms the beam pattern relevant with } \\
\text { direction and distance to guarantee a secure transmission } \\
\text { even if the legitimate user and eavesdropper locate in the } \\
\text { same direction. }\end{array}$ & 2015 \\
\hline $\begin{array}{l}\text { The eavesdropper and legitimate } \\
\text { user are in the same direction }\end{array}$ & J.g Hu et al. [118] & $\begin{array}{r}\text { Each element is randomly assigned different frequencies, } \\
\text { which can decouple the correlation between direction and } \\
\text { distance. }\end{array}$ & 2017 \\
\hline $\begin{array}{l}\text { The eavesdropper and legitimate } \\
\text { user are in the same direction }\end{array}$ & V. Bankey et al. [122] & $\begin{array}{r}\text { Each relay sends downlink traffic information in the } \\
\text { terminal direction and AN in other directions, which makes } \\
\text { the eavesdropper full of AN. }\end{array}$ & 2019 \\
\hline $\begin{array}{l}\text { CCI caused by frequency reuse } \\
\text { between beams }\end{array}$ & A. I. Neira et al. [124] & $\begin{array}{l}\text { Employed signal processing methods for efficient } \\
\text { interference suppression. Proposed advanced TPC techniques } \\
\text { to achieve adaptive coverage, dynamic traffic optimization } \\
\text { to maximize capacity within the limited frequency bands. }\end{array}$ & 2019 \\
\hline $\begin{array}{l}\mathrm{CCI} \text { caused by frequency reuse } \\
\text { between beams }\end{array}$ & L. You et al. [125] & $\begin{array}{l}\text { Establishes the massive MIMO channel model for LEO } \\
\text { SCS, simplifies the transmission designs by performing } \\
\text { Doppler and delay compensations at terminals, and obtains } \\
\text { the maximized ASLNR and ASINR. }\end{array}$ & 2020 \\
\hline $\begin{array}{l}\text { Access performance degradation } \\
\text { caused by co-frequency } \\
\text { interference in IoT supported by } \\
\text { LEO SCSs }\end{array}$ & J. Chu et al. [126] & $\begin{array}{l}\text { Adopts the NOMA scheme to support massive IoT } \\
\text { distributed over a wide range and designs the robust BF for } \\
\text { minimizing the total transmit power and mitigating } \\
\text { interference among adjacent beams. }\end{array}$ & 2021 \\
\hline
\end{tabular}

the risk of interference with GEO SCSs operating at the same frequency. Specifically, when an interference event occurs, some of the beams are briefly turned off as they cross the equator. Subsequently, when the LEO SCSs exit the GEO SCSs exclusion zone, the specific beams which were turned off are turned back on again. In the context of a hybrid beam based coverage scheme ${ }^{3}$, the authors of [48] also proposed a so-called coverage-extension method for beam drifting, which relies on expanding the wide beam to cover the serving areas of adjacent satellites. When the coverage area of a LEO satellite is overlapped by that of the adjacent satellites, one of them can be turned off to avoid the potential interference.

Given the ever-increasing deployment density of LEO megaconstellations, a spectrum crunch is imminent. Cognitive radio [139]-[141] techniques are capable of mitigating this problem. In cognitive radio networks, PUs have higher priority or legacy rights on the usage of a specific spectrum. SUs, which have a lower priority, should not cause interference to PUs. Hence SUs must have cognitive radio capabilities for adapting their communications channel access to the dynamic environments in which they operate. Explicitly, cognitive radio devices can sense, detect, and monitor the surrounding opportunities including spectrum, time, geographical space, code, as well as angle [142] and reconfigure the operating characteristics to best match those opportunities.

\footnotetext{
${ }^{3}$ There is a wide beam providing coverage for the whole service area and several spot beams for tracking users in each LEO satellite. The gain of a spot beam is designed to be much higher than that of the wide beam, hence the spot beam is provided for supporting data transmission, while the wide beam is fixed and it is suitable for control signals.
}

Cognitive radios are capable of making autonomous realtime decisions for mitigating the spectrum scarcity problem in SAGINs. The authors of [143] proposed a spectrum sensing scheme for LEO SCSs capable of mitigating the inter-system interference between GEO and LEO SCSs. Upon identifying the specific power level utilized by the GEO SCSs after differentiating the GEO signal from the interfering LEO signal and noise, the authors of [144] conceived a cognitive radio technique for improving the throughput of LEO SCSs, while guaranteeing that the signal quality of GEO SCSs can be satisfied. By applying sophisticated relaxation and approximation schemes, they significantly reduced the complexity of the related optimization problem. The authors of [145] proposed a cognitive satellite-terrestrial network relying on a distributed cooperative spectrum sensing technique by striking a tradeoff between the average throughput and the average energy consumption under specific interference constraints.

Additionally, the authors of [146] conceived a two-stage spectrum-sharing framework by combining the advantages of cognitive radio and power control techniques. This framework jointly optimizes the spectrum sensing time and the LEO SCSs transmit power with the objective of enhancing the spectral efficiency and seamless coexistence. The authors of [147] proposed a joint beam hopping and power control scheme for maximizing the throughput of LEO SCSs, while preserving the signal quality of GEO SCSs. A deep learning aided spectrum prediction method was proposed in [148] for mitigating the inter-system interference. A sophisticated combination of a convolutional neural network and of a carefully dimensioned bespoke memory was harnessed for data mining from the 
historical spectrum usage of the GEO SCSs. This technique was used for predicting the future spectral occupancy. As a further advance, a joint user pairing and power allocation scheme was designed for NOMA-based GEO and LEO SCSs in [51], where the sum rate was maximized. Furthermore, an adaptive modulation and coding method was adopted in [149] for interference mitigation. Specifically, this method adopted the angle between LEO and GEO satellites for controlling the specific choice of modulation and coding scheme, with the objective of improving the spectral efficiency of LEO SCS, while limiting the interference inflicted upon the GEO SCSs to the maximum tolerable limit.

The main results on both interference coordination are summarized in Table VI at glance.

3) Debris Removal: In practice, the LEO orbits are the most densely contaminated by space debris among all orbits, therefore LEO satellites are at the greatest risk of being hit by debris. As Wyler, the founder of OneWeb, said: "My epitaph should say 'Connect the World' instead of 'Making Orbital Garbage'." In order to exploit the space debris and effectively exploit the LEO for future exploration, we must make concerted, collaborative efforts to both prevent the generation of future debris and eliminate existing space debris. Pushing the failing or inoperative spacecraft into Earth's atmosphere and burning them down is an effective means of mitigating the generation of space debris. Researchers in Japan are even experimenting with wooden spacecraft to minimize the amount of space debris [150]. At the time of writing, many institutes are contributing to the clean-up of space debris.

Nets and Harpoons: The most famous initiative is that of European research institutions employing dedicated spacecraft to snare debris by firing harpoons and nets at them [151]. These space fishing nets are thousands of meters in diameter and are made of extremely fine wires that are woven together and strong enough to withstand the impact of space debris. The mesh is launched aboard a satellite to be deployed into space, and then it travels along Earth's orbit to sweep up space debris as it passes. Due to the gravitation of the Earth, it finally falls into the atmosphere and burns up. On September 16th, 2018, the RemoveDEBRIS satellite captured a nearby target probe that the vehicle had released a few seconds earlier, which verified the feasibility of this method [152].

Another alternative is to use space harpoons for 'hunting' satellites. Specifically, such hunting satellites employ a lidarbased guidance system to locate space debris, and a pneumatic device is designed to control the harpoon while catching moving targets. The hunting satellites could also carry tiny sub-satellites that would push the debris into the atmosphere to burn it up.

Laser 'Scavenger': A new way to deal with space debris has been proposed by Australian scientists based on adopting firing lasers from the Earth to break up space debris [153]. There are two main ways of using lasers to clean up space debris. For tiny debris, high-power laser light can be used to melt and vaporize it. Larger pieces of debris can be hit at a point, generating a backlash like a rocket jet. Thus, its course changes accordingly, and then it will drop into the Earth's atmosphere and burn up.
Robotic Arm: Japan's Aerospace Exploration Agency has also developed a robotic 'cleaner' that can use a robotic arm to firmly grasp large pieces of space debris, e.g., dead satellites, and collect them for hurling into the atmosphere to burn them up. The robot, which weighs about $140 \mathrm{~kg}$, has a robotic arm equipped with powerful magnets that can be used for slowing down space debris orbiting the Earth. However, the characteristics of most space debris are not precisely known beforehand, which results in measurement errors concerning the relative motion between the robotic arm and space debris. This makes capturing space debris complicated [154].

Giant Balloons: It is generally possible for a satellite to fire up its engines at the end of its life and head towards the Earth to burn up in the atmosphere, which would require extra fuel and eventually increase the cost of launch. The new cheaper solution is to carry a folding balloon from launch filled with helium or other gases. Once the satellite exhausted its lifespan, it could blow helium bubbles for increasing its drag through the atmosphere [155]. It takes only a year for a 37-meterdiameter balloon to drag a $1200 \mathrm{~kg}$ satellite out of its initial $830 \mathrm{~km}$ orbit and to crash it into the Earth's atmosphere to burn it up.

'Suicide' Satellite: The aforementioned methods of removing space debris, like using nets, harpoons, robotic arms, or lasers, are costly. Scientists in the UK developed a lowcost device called Cubic Sail to clean up space debris [156]. CubeSail is a 'suicide' micro-satellite, weighing just $3 \mathrm{~kg}$, that can be launched into space. Once locked on to its target, it would deploy its kite-like solar sail, attach itself to space debris and slow its flight. Eventually, they will perish.

Table VII compares the advantages and disadvantages of these debris removal techniques. However, these solutions are currently in the design or experimental phase, and more engineering efforts are required to put these ideas into practice.

\section{B. Passive Security Countermeasures}

In contrast to advanced security countermeasures, passive security countermeasures must first directly face security threats and then they are spurred into action to mitigate their impact as much as possible. Passive security countermeasures tend to rely on traditional mathematics-based encryption, SS techniques, Terahertz (THz) and space-based laser communications, and SEU mitigation measures, which will be detailed below.

1) Traditional Mathematics-based Encryption: At the time of writing, the traditional encryption techniques relying on excessive-complexity mathematical operations are widely used in the ground facilities of SCSs for improving the security. The five-layer transmission control protocol/Internet protocol model of SCSs is comprised of the application layer, transport layer, network layer, media access control layer, and physicallayer [157]. The above-mentioned traditional mathematicsbased encryption techniques are mainly employed in the upper four layers above the physical-layer for maintaining confidential and secure transmissions. Naturally, they have to satisfy the demanding authentication, integrity, and freshness specifications of the system to cope with spoofing jamming, 


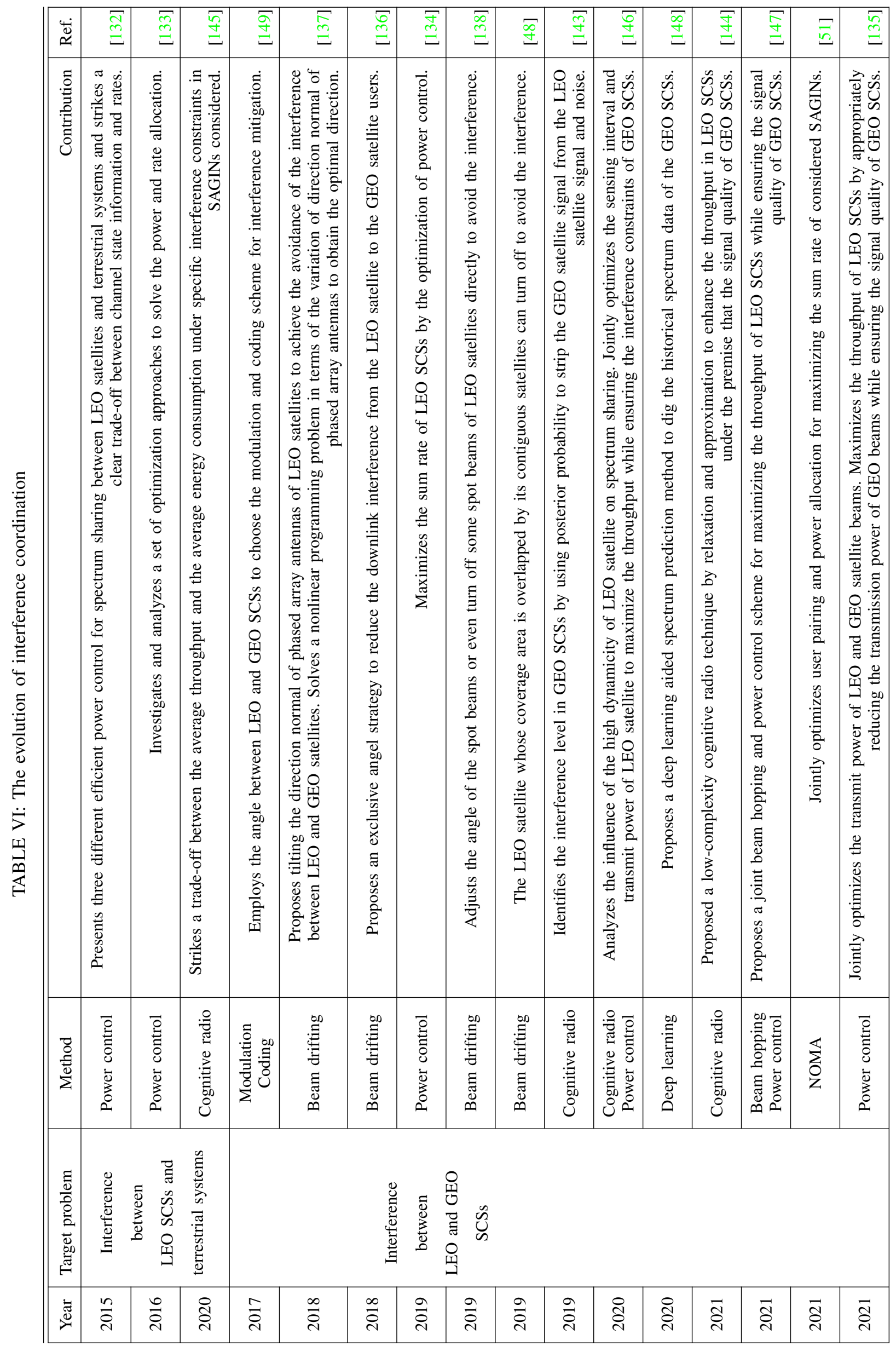


TABLE VII: A table comparison of debris removal techniques

\begin{tabular}{|c|c|c|}
\hline Project & Advantages & Disadvantages \\
\hline \multirow[t]{2}{*}{ Nets and harpoons } & $\begin{array}{l}\text { Able to handle irregular and spining debris } \\
\text { compared to a robotic arm }\end{array}$ & Nets is not able to be reused \\
\hline & Nets prevent further debris generation & $\begin{array}{r}\text { Smashing large space debris by harpoons may generate } \\
\text { further debris }\end{array}$ \\
\hline \multirow{3}{*}{ Laser 'scavenger' } & Effective for small space debris & May burn up the debris causing extra debris \\
\hline & Able to dexterously handle tumbling debris & $\begin{array}{l}\text { Large amount of beam energy is because it is hard to } \\
\text { generate a small beam at a long distance }\end{array}$ \\
\hline & Able to be reused & Sophisticated target detection and acquisition system \\
\hline \multirow{2}{*}{ Robotic arm } & Able to grasp space debris firmly & Sophisticated control \\
\hline & Able to be reused & Easily penetrated by debris, especially sharp debris \\
\hline \multirow[t]{2}{*}{ Giant Balloons } & $\begin{array}{l}\text { Effective large space debris such as failing or } \\
\text { inoperative spacecraft }\end{array}$ & Easily penetrated by debris, especially sharp debris \\
\hline & Preventing further debris generation & Slow response because of balloon inflation \\
\hline \multirow{2}{*}{ 'Suicide' Satellite } & Preventing further debris generation & Not able to be reused \\
\hline & Low cost & Suitable for larger debris \\
\hline
\end{tabular}

node compromise, and hijacking, as illustrated in Fig. 16. The associated aspects are further detailed below.

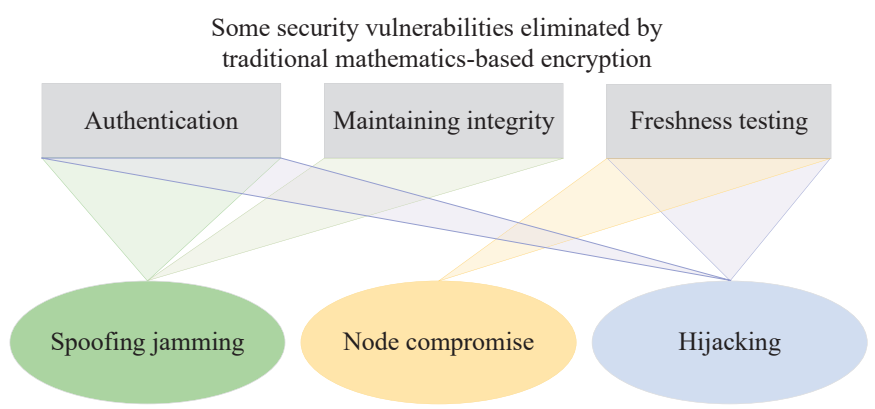

Fig. 16: The relationship between security vulnerabilities and requirements.

Authentication: Authentication refers to distinguishing legitimate users from illegal users. Because of the shared wireless communication link, each user has to reveal its identity. Authentication must be completed prior to communication, hence it can protect the system against hijacking and spoofing jamming [83], [92].

Maintaining Integrity: Integrity means that the information remains accurate and reliable during the transmission process, and has not been tampered with or modified by eavesdroppers [66]. In other words, the information received must be the same as the transmitted information. It is difficult for the adversary to accurately forge the original information. Hence including integrity detection mechanisms is capable of alleviating spoofing jamming [158].

Freshness Testing: A compromised node is capable of successful authentication because it has the secret keys of a legitimate node [92]. A compromised node running malicious software has all the distinctive characteristics of a legitimate node [66]. Freshness testing employs code patches for distinguishing compromised nodes from legitimate nodes [65]. Dynamic secret key generation based on freshness testing is capable of preventing hijacking attacks [159].

2) SS Techniques: SS techniques have been routinely adopted as one of the secure techniques in military communications for more than 70 years [160], where the transmitted signal is spread to a much wide bandwidth than the information bandwidth. The common SS techniques include DSSS, Frequency Hopping Spread Spectrum (FHSS), and MultiCarrier Direct Sequence Spread Spectrum (MC-DSSS). Unless the eavesdropper steals the random Frequency Hopping (FH) pattern or spreading code, it fails to detect the confidential information [161].

Again, DSSS has been widely used in satellite communications [162]. Whenever interference contaminates the desired signal, the receiver correlator spreads the interference to the entire bandwidth after despreading, because the interference and the local pseudo-noise code are uncorrelated. By contrast, the desired signal is despread back to its original narrower bandwidth. The SNR of the baseband data increases after despreading by a factor of the Processing Gain (PG). By contrast, the PSD of interference remains low in the baseband. Hence, the anti-interference ability also depends on the PG. However, the payload rate is given by the ratio of the bandwidth and the spreading factor, which explicitly indicates a throughput versus interference level trade-off in LEO SCSs. More specifically, when the interference is strong, the DSSS sequence length should be increased to improve the anti-interference capability controlled by its PG, hence leading to throughput reduction and vice versa.

Furthermore, FHSS constitutes another popular antiinterference technique. In contrast to DSSS, the FHSS transceiver continuously jumps from one sub-carrier frequency to another during transmission according to the SS code. Hence the FHSS signal bandwidth may be composed of discontinuous frequency bands, and it is often combined with cognitive radio techniques to avoid interference at locations subject to severe interference, whilst relying on adaptive frequency hopping. 
Hopping across multiple frequencies within a single symbol leads to the concept of Fast Frequency Hopping Spread Spectrum (FFHSS). More explicitly, the dwell time of each hop is shorter than the symbol duration and multiple frequency hops are completed within a single symbol duration, leading to strong anti-interference capability. FFHSS may rely on lowcomplexity non-coherent dehopping and demodulation methods, but this results in a substantial loss of SNR [163], [164]. By contrast, the coherent reception of FFHSS exhibits better performance [165], at a substantially increased complexity.

Compared to DSSS, the bandwidth of MC-DSSS systems [166] is wider due to the use of multiple carriers, when SS having the same length is adopted. More explicitly, the bandwidth is expanded proportionately by the number of subcarriers. Additionally, this waveform could also be combined with spectrum sensing for further improving the level of security. The results of spectrum sensing may be beneficially combined with adaptive sub-carrier activation schemes, and interference suppression arrangements in a flexible manner, as shown in Fig. 17. Hence there is a trade-off between the security and the integrity. Explicitly, the transmitter is capable of intelligently adjusting the center frequency as well as the transmit power of its sub-carriers for mitigating the interference, which improves the system's integrity. However, these sub-carriers remain more vulnerable to eavesdropping. Conversely, these sub-carriers of the transmit waveform could also be actively hidden in some of the existing interference for improving the security, which however makes the system more vulnerable to these interferences.

To further illustrate this trade-off, the dependence of the Bit Error Rate (BER) on the specific fraction of the total frequency band buried in the interference is plotted in Fig. 18. As shown in Fig. 18, the BER degrades as the fraction of the total frequency band concealed in the interference increases from $10 \%$ to $40 \%$.

3) Non-SS Interference Suppression Techniques: When the interference power exceeds the maximum tolerance level of the SS receiver, the SS system has to resort to employing dedicated interference suppression algorithms, such as temporal domain adaptive filtering [98] and transform domain adaptive filtering [99].

Temporal domain adaptive filtering algorithms are suitable for narrowband interference suppression. The Least Mean Square (LMS) [98], [167] algorithm is a popular design option due to its low complexity. The basic idea behind LMS algorithm is to mimic a causal Wiener filter by updating the filter weights until the least mean square of the error signal is approached. It is a stochastic gradient descent method, which means that the filter weights are only adapted based on the error at the current symbol instant. For a standard LMS algorithm, the convergence speed is determined by the step size parameter $(\mu)$, which may be gradually reduced upon approaching convergence to the minimum.

On one hand, the higher the value of $\mu$, the faster the weights converge. Hence we can promptly track and mitigate the fluctuating interference. On the other hand, the higher $\mu$, the higher the variance of the weights will be, which affects the performance of interference mitigation. Therefore, the realization of the LMS algorithm requires a trade-off, as illustrated in Fig. 19.

By contrast, transform domain adaptive filtering is capable of promptly tracking the fluctuation of narrowband interference without an iterative process [168]. Transform domain adaptive filtering processes the received signal in the frequency domain. Briefly, it identifies the interference and carries out the band-pass filtering, before transforming the signal back to the temporal domain.

4) THz and Space-based Laser Communications: The frequency allocations of several commercial LEO satellite constellations are shown in Fig. 20. Observe that many LEO satellites operate in the decimeter wave and centimeter wave bands, such as Iridium and Globalstar. As the time of writing, the Millimeter Wave (mmWave) band is attracting research attention as a benefit of its rich spectral resources [169]. Many LEO satellite manufacturers such as Boeing, Starlink, and OneWeb sought permission to launch satellites operating in the 50.2-52.4 Gigahertz (GHz) bands [12], [15], [170]. However, these frequency resources are becoming congested. A potential solution is to increase the operating frequency to the $\mathrm{THz}$ or even optical bands. Thanks to the development of device and communication technology, these emerging bands are gradually entering commercialization [171], [172].

THz communications: The $\mathrm{THz}$ band has a vast amount of available bandwidth, which has to be further explored. The radio frequencies above $100 \mathrm{GHz}$ are largely untapped for specific applications by the ITU, hence they might become available for SCSs. Fig. 21 shows the application scenarios of THz communications in LEO SCSs, including THz-based Inter-satellite Links (ISLs), THz-based smart wearable devices, and THz-based secure short-distance transmissions.

Although the high path loss of the $\mathrm{THz}$ band only facilitates short-range RF communications, this has the benefit of limiting the eavesdropping opportunities in secure communications. Additionally, the energy of $\mathrm{THz}$ photons is low, hence mitigating the biological effects. This suggests that sensors operating in the THz band can be embedded in the human body to transmit medical information to smart wearable terminals [173], [174].

Satellites are subject to atmospheric interference that prevents the use of microwave bands. The employments of $\mathrm{THz}$ communications for ISLs [175], which operate above the Earth's atmosphere, could be an attractive alternative. According to [176], THz transmitters and receivers could be designed for circumventing the disadvantages of the microwave bands. Although the attenuation of the $\mathrm{THz}$ band is high, this may potentially be compensated by large-scale antennas used for BF on a space-borne payload. The beamwidth of the largescale antennas in the $\mathrm{THz}$ band is narrower than that of common microwave ISLs, which enhances their ability to resist eavesdropping.

A summary of the state of the art of successful wireless data transmissions, in terms of data rate versus link distance, is presented in Fig. 22. Observe that the longest communication distance was $21 \mathrm{~km}$ at $140 \mathrm{GHz}$ [177], which is insufficient for ISLs. Therefore, a large antenna array and high-power devices operating in the $\mathrm{THz}$ band should be developed to overcome 


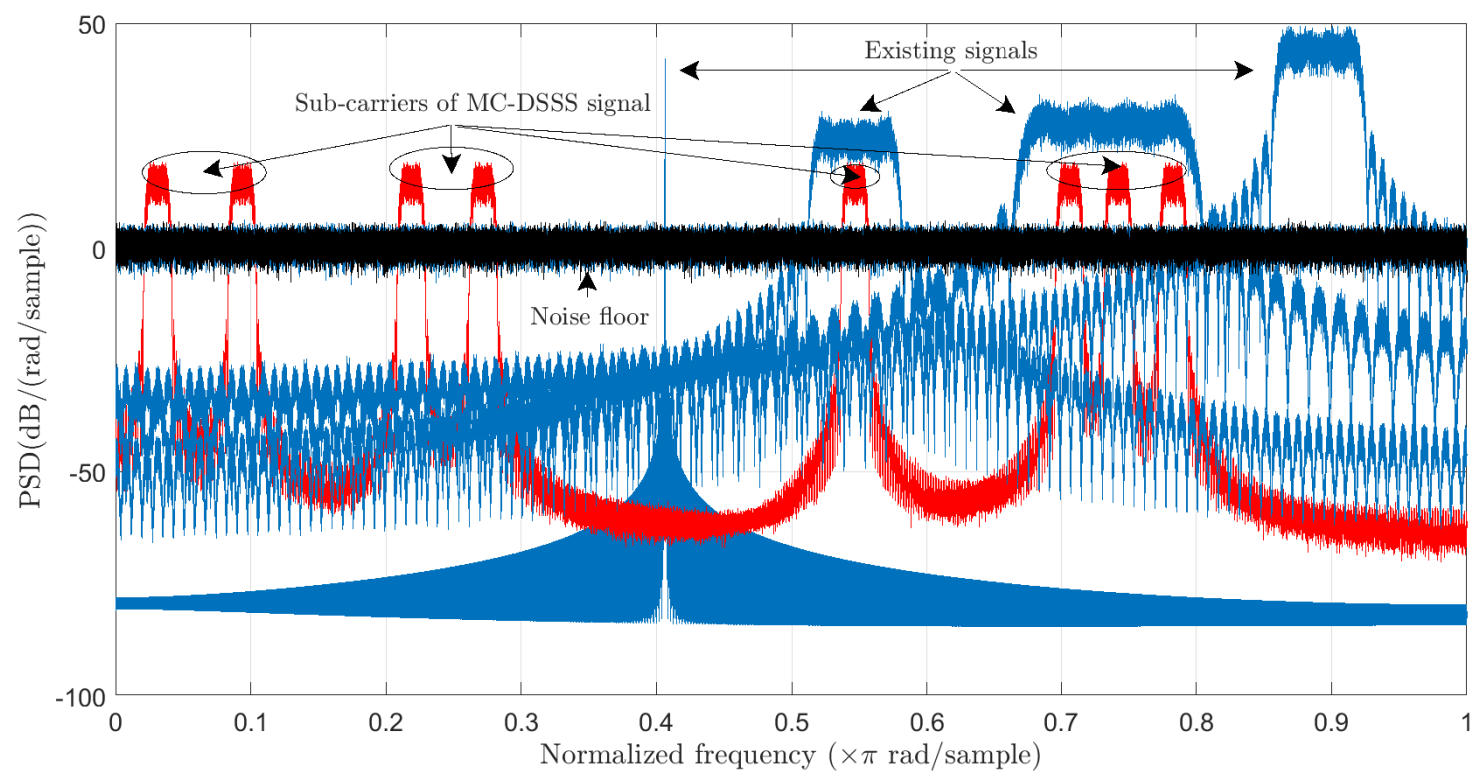

Fig. 17: The trade-off between security and integrity in MC-DSSS systems.

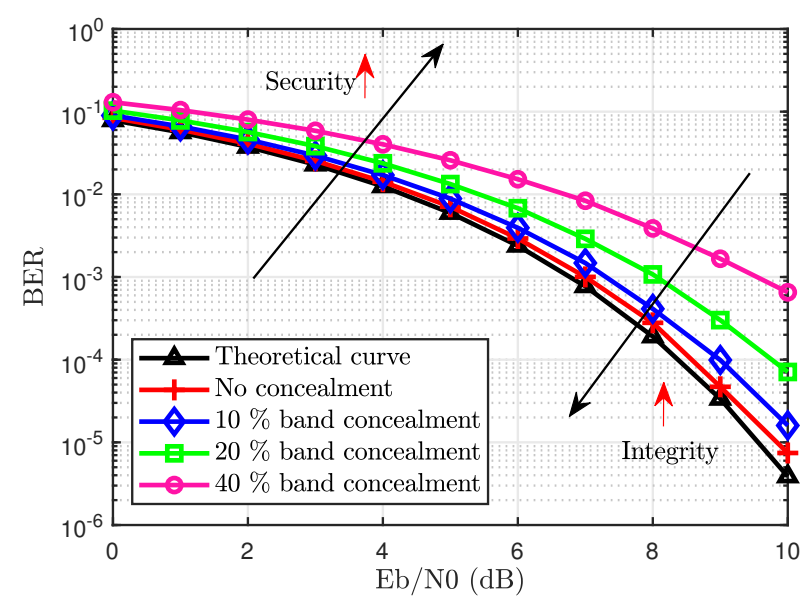

Fig. 18: The variation of BER with band concealed in existing interference.

the extremely high propagation loss and power limitations of the space-borne transceivers in harsh operating environments.

\section{Space-based laser communications:}

The laser band is far above the electromagnetic spectrum, thus it has a strong anti-interference capability. Laser communications cannot be detected by spectrum analyzers or RF meters, since the laser beam is highly directional, which makes it a strong candidate for ISLs and cross-layer links [192]. Additionally, laser offers several advantages over microwave communications in terms of size, weight, and power dissipation compared to the mmWave band under the same data rate conditions [193], [194].

Many research institutions across the world have conducted numerous experiments, which are summarized in Table VIII at glance. Additionally, Starlink tested 'space lasers' between two

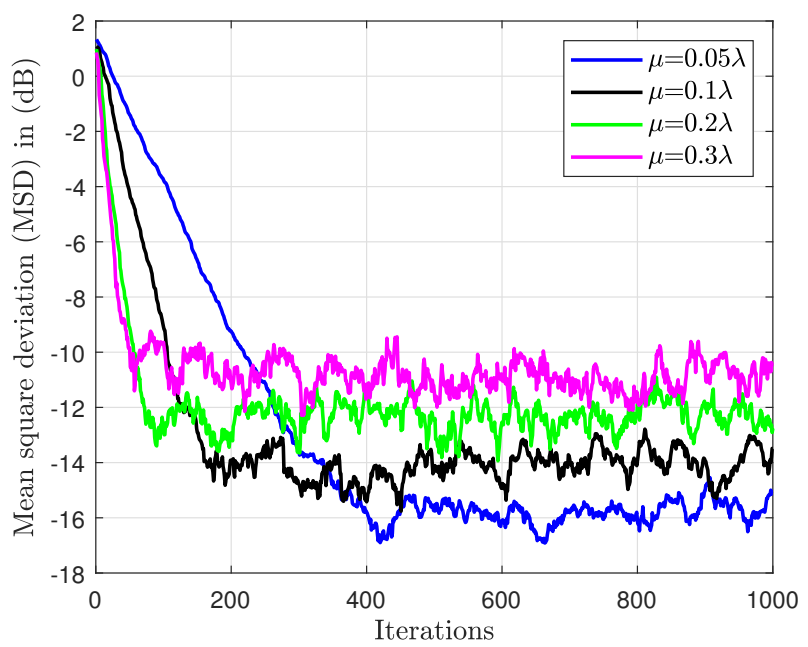

Fig. 19: The comparison between the convergence performance and the NWD of weights. The number of taps

is 4. $\mu$ is given as $0<\mu<\frac{2}{\lambda}$, where $\lambda$ is the greatest eigenvalue of the autocorrelation matrix

$$
R=E\left\{X(n) X^{H}(n)\right\} \text {. }
$$

satellites, relaying hundreds of Gbytes of data in Sep. 2020. At the time of writing, Starlink is engaged in rolling out further laser cross-links amongst their satellites for minimizing the number of ground facilities and for extending the coverage to remote areas [195], [196].

Although space-based laser communications are not affected by the atmosphere and weather, the high velocity and the jitter of the space-borne payload [214] make the alignment and focus of the beam a challenge. Furthermore, significant Doppler frequency shifts may be observed by the spaceborne laser terminals in the 'reverse seam', as illustrated in 


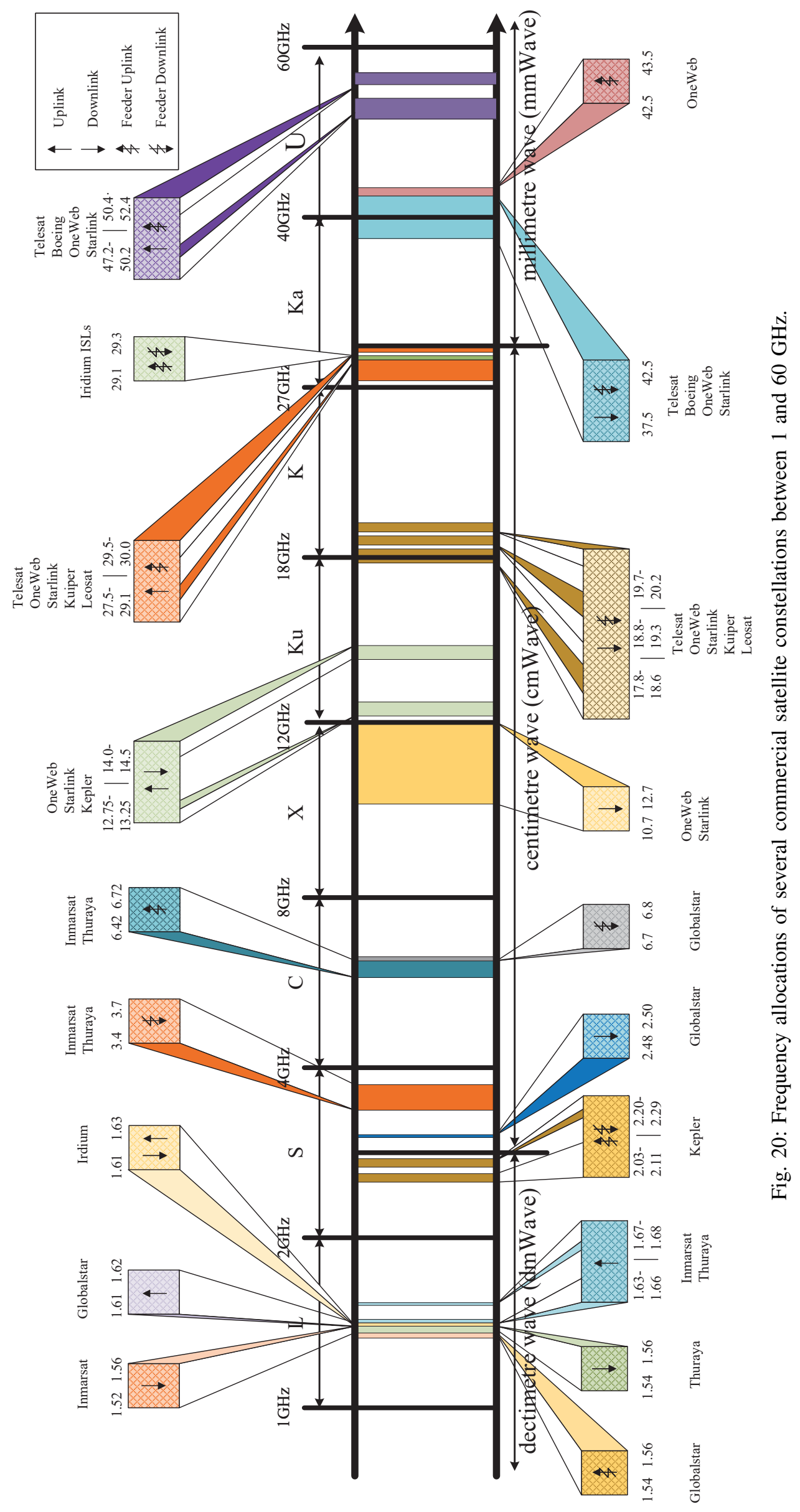


TABLE VIII: The evolution of space-based laser communications

\begin{tabular}{|c|c|c|c|c|c|c|c|}
\hline Year & Project & Type & Country/Region & Data rate (Mbps) & Modulation & Distance $(\mathrm{km})$ & Ref. \\
\hline 2001 & SILEX & GEO-LEO & Europe & 50 & IMDD & 45000 & [197] \\
\hline 2006 & OICETS & LEO-OGS & Japan & 50 & IMDD & 610 & [198] \\
\hline 2010 & $\begin{array}{l}\text { TerraSAR- } \\
\text { X/NFIRE }\end{array}$ & $\begin{array}{l}\text { LEO-OGS } \\
\text { LEO-LEO }\end{array}$ & Europe & 5625 & BPSK & $\begin{array}{c}500-1000 \\
1000-5000\end{array}$ & [199] \\
\hline 2011 & BTLS & LEO-OGS & Russia & 125 & IMDD & 400 & [200] \\
\hline 2013 & LLCD & Lunar-OGS & US & 622 & PPM & 400000 & [201] \\
\hline 2013 & Alphasat & GEO-LEO & Europe & 1800 & BPSK & 45000 & [202] \\
\hline 2014 & OPALS & LEO-OGS & US & 50 & IMDD & 400 & [203] \\
\hline 2014 & SOTA & LEO-OGS & Japan & 10 & OOK/IMDD & 642 & [204] \\
\hline 2016 & MICIUS & LEO-OGS & China & 5120 & DPSK & 1500 & [205] \\
\hline 2016 & OCSD & LEO-OGS & US & 200 & IMDD & 450 & [206] \\
\hline 2017 & VSOTA & LEO-OGS & Japan & 10 & - & 1000 & [207] \\
\hline 2017 & SJ-13 & GEO-OGS & China & 4800 & IMDD & 36000 & [208] \\
\hline 2020 & EDRS-C & GEO-LEO & Europe & 1800 & BPSK & 45000 & [209] \\
\hline 2020 & SJ-20 & GEO-OGS & China & 10000 & OOK/BPSK/QPSK & 36000 & [210] \\
\hline 2023 & CubeSOTA & $\begin{array}{l}\text { GEO-LEO } \\
\text { LEO-OGS }\end{array}$ & Japan & 10000 & DPSK & $\begin{array}{c}39693 \\
1103\end{array}$ & [211] \\
\hline 2025 & EDRS-D & GEO-GEO & Europe & $3600-10000$ & BPSK & 80000 & $\begin{array}{l}\text { [208], } \\
\text { [212] }\end{array}$ \\
\hline 2025 & ScyLight & $\begin{array}{l}\text { GEO-LEO } \\
\text { LEO-OGS }\end{array}$ & Europe & 100000 & - & $\overline{80000}$ & $\begin{array}{l}\text { [208], } \\
{[213]}\end{array}$ \\
\hline
\end{tabular}
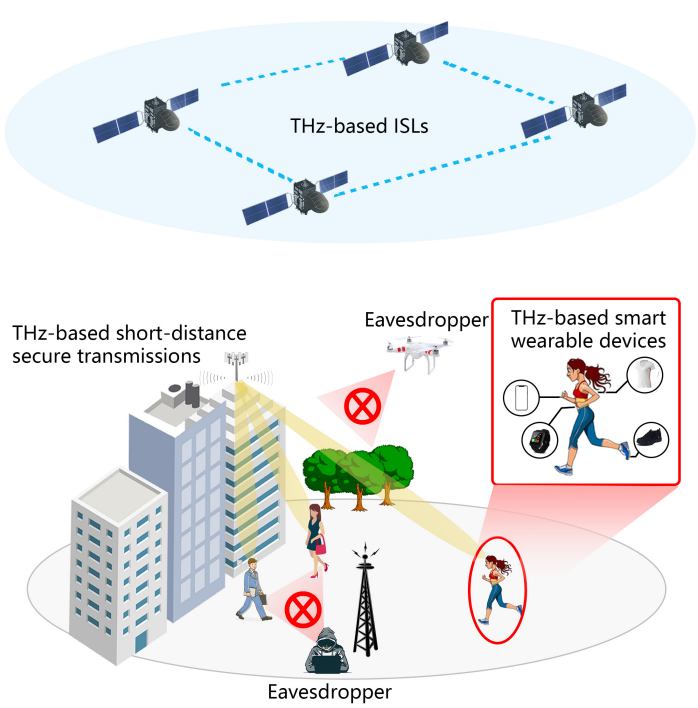

Fig. 21: Some THz application scenarios.

Fig. 23(b), which is formed between two orbits evolving in the opposite directions. The authors of [215] analyzed the Doppler frequency shift of LEO SCSs relying on laser links. Inadequate Doppler frequency shift compensation results in loss of data and frequency synchronization issues at the receiver.

5) SEU Mitigation Measures: SEU mitigation is an engineering problem involving advanced chip technology and different forms of redundancy for ensuring the reliable operation of the space-borne payload in harsh space environments. The formulation of SEU mitigation measures usually obeys the process shown in Fig. 24. The time-invariant functions should be implemented by ASICs, while the programs that have to be upgraded or iterated should be implemented using FPGAs because of their flexibility.

For the program implemented in FPGA, usually TMR is adopted for preventing the impact of SEU [216]. Briefly, TMR is a fault-masking scheme based on feeding the outputs of three identical copies of the original program module to a majority voter. If the output of the three modules is the same, the system will be regarded to operate normally. If any faults occur in one of the modules, the other modules can mask the fault. Thus, TMR can efficiently prevent single faults from propagating to the output.

However, there is a trade-off between resource consumption and reliability. The resource consumption of TMR is three times that of the original program module. Hence, designers usually apply the TMR philosophy only to the key part of the program, such as the control part.

The parts operating without TMR require the periodical refreshing technique of [217] to correct errors by refreshing the program without interrupting its execution as detailed in [218]. However, the block Random Access Memory (RAM) used in FPGAs will be initialized during the periodical refreshing operation, when its real-time state is lost. Hence the block RAM should also adopt TMR for mitigating the impact of SEU [219]. In a nutshell, the combination of partial TMR and periodical refreshing should be adopted for ensuring reliable 


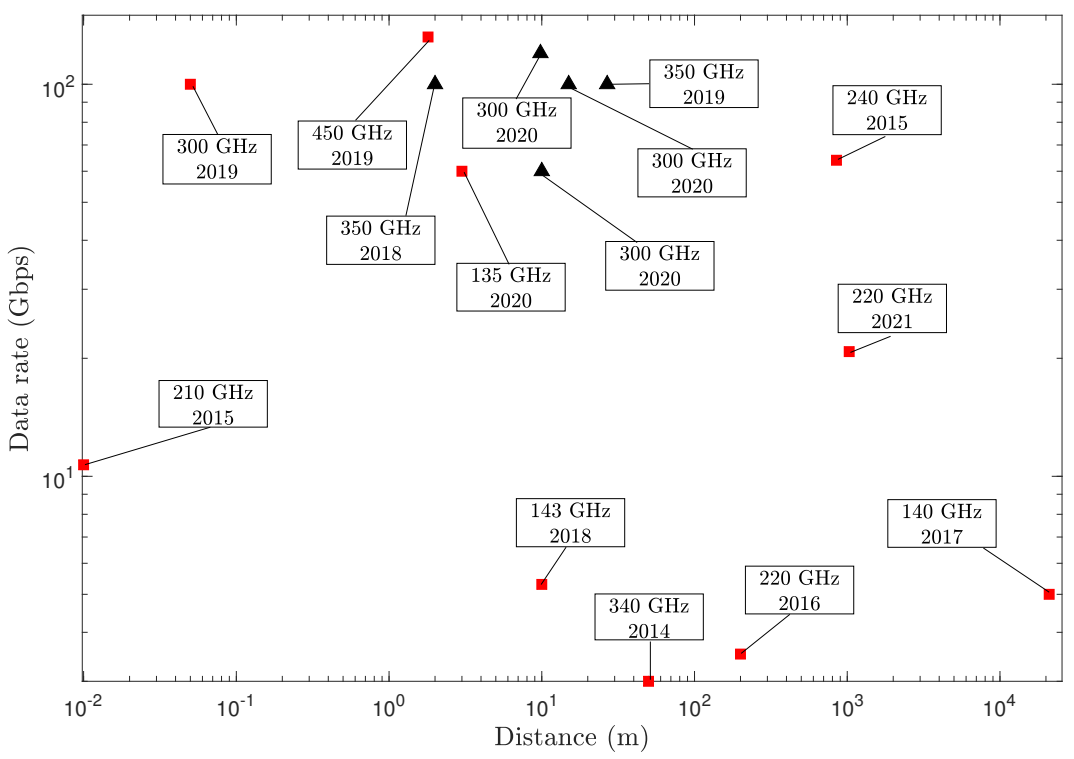

Fig. 22: State of the art in $\mathrm{THz}$ wireless communication links operating at carrier frequencies above $100 \mathrm{GHz}$. Experimental results, including BER measurements, are defined as real-time demodulation [177]-[186], indicated by squares. By contrast, the triangles indicate non-real-time demodulation [187]-[191].

and stable operation.

\section{Future TRends}

Given the rapid developments of quantum technology, Artificial Intelligence (AI), IRS, and blockchain, they have great potential to cope with the security vulnerabilities of LEO SCSs. This section will address the new opportunities in the security of LEO SCSs and highlight potent research topics for stimulating future research.

\section{A. Secure Quantum Communications}

The conceptually simplest encryption method relies on generating a pseudo-random secret key and then taking the modulo-two function of the key and the information to be encrypted, which is termed as plain text. Naturally, the key has to be as long as the data sequence to be transmitted, which implies imposing an overhead of $100 \%$.

Then the resultant so-called ciphertext may be transmitted from the source to the destination over a public channel. Given the knowledge of the secret key, the receiver can recover the original plaintext using the secret key. Since the key must remain confidential for the communications of the two parties, it must be shared between them over a secure channel.

The family of legacy cryptography schemes was conceived under the assumption that it would require an excessive amount of time even upon using the most powerful computers by the eavesdropper to infer the key. However, given the threat of powerful quantum computers, it is no longer safe to rely on the above-mentioned antiquated assumption.

Similarly simple principles may be used in Quantum Key Distribution (QKD) systems for the encryption/decryption process, but the negotiation of the secret key relies on a quantum channel as well as on an insecure public channel plus an authenticated public channel. The family of satellitebased QKD systems was richly characterized in [220], along with diverse satellite channels using detailed examples, hence here we dispence with elaborating on them further. We note however that a detailed list of future research ideas on QKD was also provided. Before concluding our discussions of QKD we note that in 2016 the so-called MICIUS experiment demonstrated the feeasibility of QKD over a satellite link, bridging a distance of $1200 \mathrm{~km}$ using free-space optical links [221]. However, as the terminology suggests, QKD remains a key-negotiation and distribution protocol used by classical systems.

By contrast, quantum secure direct communications (QSDC) [222] is a fully-fledged quantum communication protocol, which has hence enjoyed a rapid evolution, as documented in [223]-[230].

\section{B. 3D Virtual Arrays}

Due to the physical constraints on the weight, size, and energy consumption of LEO SCSs, only a limited number of space-borne antennas can be used on each satellite, which limits the array gain and interference suppression capability. For example, it is difficult to suppress interference, when the legitimate user and the interference are in the same direction. A potential solution is to improve the performance through the collaboration of multiple satellites. Given the proliferation of LEO mega-constellations, these LEO satellites are capable of forming a 3D virtual array.

To elaborate briefly, 3D virtual arrays can be formed by sharing antennas among all cooperating satellites in the orbits for better interference mitigation and information transmission. 


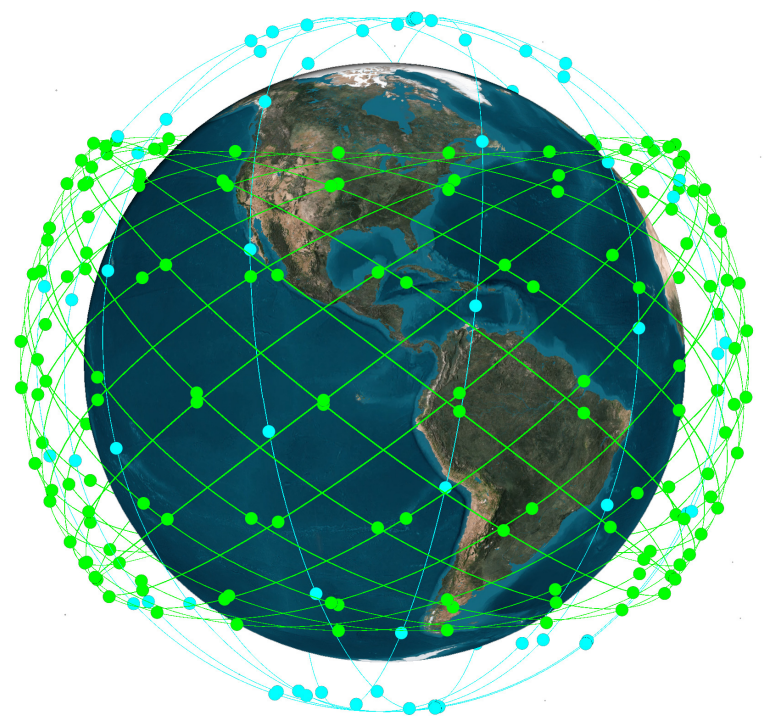

(a) Lightspeed's LEO satellite constellation.

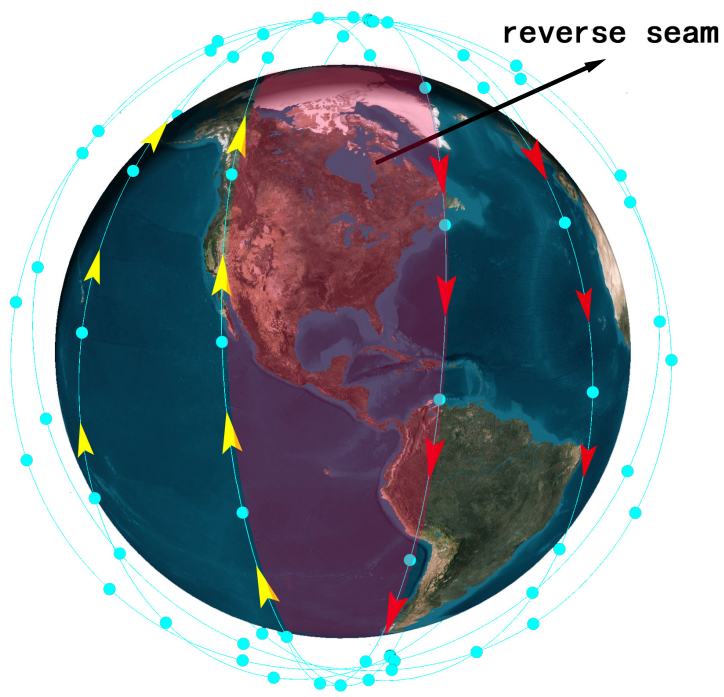

(b) Description of the 'reverse seam' in polar orbits

Fig. 23: Lightspeed's constellation and a snapshot of the 'reverse seam' in the constellation.

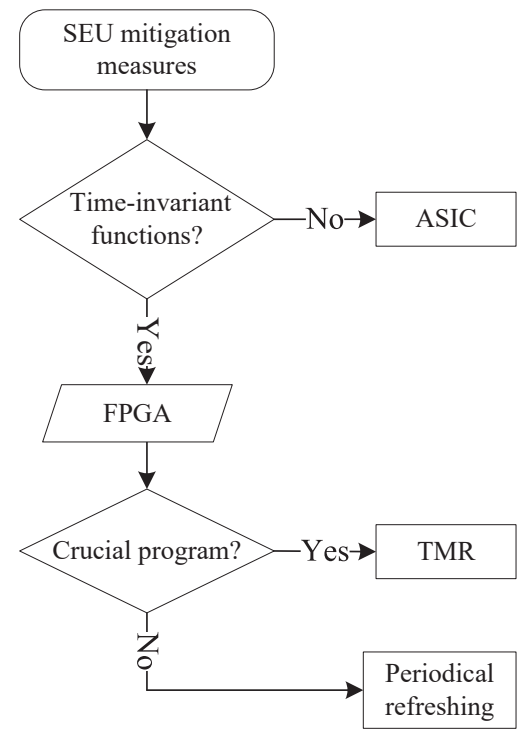

Fig. 24: A flow chart of SEU mitigation measures.

Even when the legitimate user and the interference are in the same direction within the coverage of a satellite, other satellites in the virtual array can still perform interference suppression by creating 3D virtual transmission nulls. By combining satellites in the array, there is a great potential to achieve performance gains.

Again, the 3D virtual arrays are capable of substantially improving the received SNR with the aid of the weighted combining of the signals transmitted by a specific terminal in the uplink, provided that the frequency and phase shifts as well as delays amongst the satellites are accurately estimated and compensated. However, owing to the high velocity of LEO satellites, the time-variant frequency and phase shifts as well as delays pose a serious challenge in terms of carrying out the aforementioned weighted combining at multiple satellites.

\section{AI-based Security Measures}

AI [231] will facilitate the flexible intelligent interference suppression [232]. The ability to learn is of pivotal importance, since there are large discrepancies in satellite coverage quality across the globe. Hence the prevalent traffic flow, service type, and CIR in the coverage area have to be recorded, learned, and analyzed. As a benefit, the communications resources can be adjusted in real time according to the predicted service distribution within the satellite's coverage area in real time. Consequently, more resources are allocated in areas with heavy traffic and vice versa.

However, given the limited resources of space-borne payloads, most of the AI algorithms tend to rely on the abundant computing resources of the ground facility, which inevitably the real-time performance of the system. Hence there is a pressing need for the concept of low-complexity space-based AI algorithms, which are capable of operating in the face of uncertainty.

\section{Space-based Blockchain}

At the time of writing, both the satellites of LEO megaconstellations and the terminals are centrally managed by the ground facility, which poses a serious security challenge. Blockchain [233] has the benefits of both decentralized tamper-resistance and anonymity. Hence it has been proposed for next-generation networks [234], [235]. The blockchain philosophy relies on a decentralized database that is jointly maintained by multiple parties and uses sophisticated encryption. 
Space-based blockchain can also enhance the spatial situational awareness, which helps avoid space debris in LEO. By using blockchain, Surdi et al. [236] investigated a selforganized decentralized ground facilities and satellites with the objective of avoiding deficiencies of the current satellite and debris tracking systems.

On the other hand, user data can be stored, relayed, securely registered, and updated in each LEO satellite node, which substantially enhances the data security [237]. Thanks to the associated distributed secure management, illegal nodes that drop packets can be reliably detected. Additionally, the authors of [238] revealed that sharing location information in the blockchain can prevent spoofing jamming. However, the data stored in the blockchain is public, thus illegal users may be able to breach privacy through data mining. Therefore, how to improve the privacy protectional ability of blockchain remains an open issue.

\section{E. IRS-Aided Secure Transmission}

Again, there is a high path loss between the LEO satellites and the ground terminals owing to the long transmission distance. Additionally, when the elevation angle ${ }^{4}$ of the ground terminal is low, tall buildings and other structures often block the wireless signals. To circumvent this problem, IRSs constitute promising range expansion techniques, which impose carefully optimized phase shifts on their incident signal, as detailed in [239]-[241].

To expound a little further, the authors of [242] conceived a beneficial scheme for improving the received SNR at the ground terminals receiving at a low elevation angle by deploying IRSs on another satellite having a higher elevation angle wrt the ground terminal considered. Furthermore, the joint optimization of the active transmit beamformer and of the passive reflection-based beamforming was proposed for maximizing the received SNR. As a further evolution, by exploiting the predictable mobility of LEO satellites, the authors of [243] developed a continuous time model and optimized the configuration of IRSs with respect to the timevariant received SNR, Doppler frequency shifts, and delay.

However, numerous new opportunities are provided by the IRSs in terms of safeguarding the security of wireless communication systems [240]. Briefly, the IRs and transmitter cooperation based BFs are capable of enhancing the signal at the legitimate users and degrading the signal received by the eavesdroppers [244]. Yu et al. [245] conceived an $\mathrm{AN}$-assisted beamforming scheme for sum-rate maximization, while limiting the maximum information leakage.

However, there is a paucity of literature on the security of LEO SCSs relying on IRSs. Hence, how to exploit the unique advantages of IRSs for enhancing the security of LEO SCSs and what benefits they will provide for the LEO SCSs' security requires substantial further research in the face of their timevariant received SNR, Doppler frequency shifts, and delay.

\footnotetext{
${ }^{4}$ The elevation angle represents the angle between the satellite and the horizontal tangetial line touching the earth' surface.
}

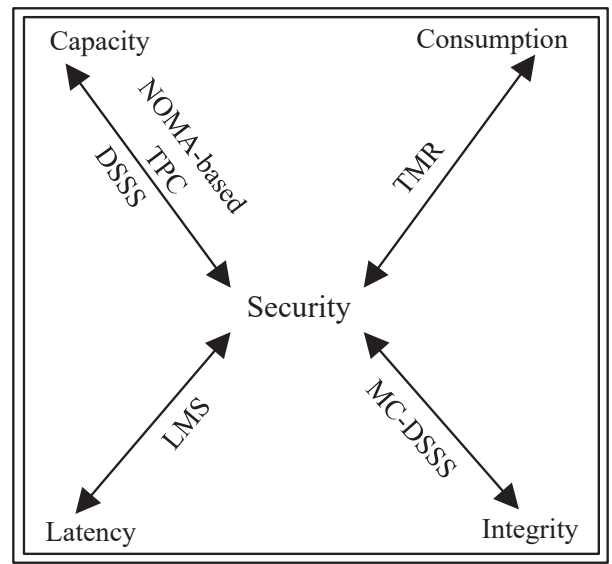

Fig. 25: Relationship of the countermeasures mentioned in this paper for security, resulting in different design trade-offs.

\section{Summary and Design Guidelines}

In this section, we provide some general design guidelines for secure LEO SCSs based on the associated trade-offs and summarize the main points' take-away messages.

\section{A. Summary}

LEO SCSs have attracted increasing attention as a benefit of their seamless global coverage with low latency. However, there are many open issues in the course of exploiting the full potential of LEO SCSs, including their security issues. Due to inherent characteristics such as special location, high mobility, and so on, LEO SCSs suffer several severe security challenges. In this paper, We mainly discussed their security vulnerabilities and corresponding security countermeasures.

We analyzed the security vulnerabilities of LEO SCSs based on their inherent characteristics, which include passive eavesdropping, active eavesdropping, interference contamination, SEU, and space debris. For mitigating these security vulnerabilities, we then introduced and summarized some corresponding security countermeasures, which can be divided into active and passive security countermeasures. Finally, we highlighted secure quantum communications, 3D virtual arrays, AI-based security measures, space-based blockchain, and IRS enabled secure transmission as security countermeasures for employment in future LEO SCSs, which require substantial future research.

\section{B. Design Trade-Offs in Secure LEO SCSs}

By identifying the specific cause of security vulnerabilities in LEO SCSs, they can potentially be mitigated. Specifically, recall from Fig. 6 that we highlighted the security versus integrity trade-off encountered in LEO SCSs without using any secrecy coding, since increasing the transmit power improves the integrity but inevitably increases also the eavesdropping probability. Increasing the frequency reuse factor for improving the capacity will also inevitably increase the probability of $\mathrm{CCI}$, thereby increasing the BER, ie. degrading the integrity.

A series of security countermeasures were presented, but most of them are subject to design trade-offs, as intimated in 
TABLE IX: List of acronyms

\begin{tabular}{|c|c|c|c|}
\hline Acronyms & Definitions & Acronyms & Definitions \\
\hline $3 \mathrm{D}$ & Three-dimensional & LoS & Line of Sight \\
\hline AI & Artificial Intelligence & MAI & Multiple Access Interference \\
\hline AN & Artificial Noise & MC-DSSS & Multi-Carrier Direct Sequence Spread Spectrum \\
\hline ASC & Average Secrecy Capacity & MEO & Medium Earth Orbit \\
\hline ASIC & Application Specific Integrated Circuit & MIMO & Multiple Input Multiple Output \\
\hline ASINR & Average Signal to Interference Plus Noise Ratio & MmWave & Millimeter Wave \\
\hline ASLNR & Average Signal to Leakage Plus Noise Ratio & $\mathrm{ms}$ & Milliseconds \\
\hline BER & Bit Error Rate & NASA & National Aeronautics and Space Administration \\
\hline $\mathrm{BF}$ & Beamforming & NOMA & Non-orthogonal Multiple Access \\
\hline $\mathrm{CCI}$ & Co-channel Interference & OGS & Optical Ground Station \\
\hline CIR & Carrier to Interference Ratio & PG & Processing Gain \\
\hline COTS & Commercial Off The Shelf & PSD & Power Spectral Density \\
\hline DRL & Deep Reinforcement Learning & PU & Primary User \\
\hline DSSS & Direct Sequence Spread Spectrum & QKD & Quantum Key Distribution \\
\hline ESA & European Space Agency & RAM & Random Access Memory \\
\hline FDA & Frequency Diverse Array & $\mathrm{RF}$ & Radio Frequency \\
\hline $\mathrm{FH}$ & Frequency Hopping & RFDA & Radom Frequency Diverse Array \\
\hline FHSS & Frequency Hopping Spread Spectrum & RRM & Radio Resource Management \\
\hline FFHSS & Fast Frequency Hopping Spread Spectrum & SAGIN & Space-air-ground Integrated Network \\
\hline FPGA & Field Programmable Gate Array & SCS & Satellite Communication System \\
\hline GEO & Geostationary Earth Orbit & SDR & Software Defined Radio \\
\hline $\mathrm{GHz}$ & Gigahertz & TMR & Single Event Upsets \\
\hline GPS & Global Position System & TPC & Transmit Precoding \\
\hline IoT & Internet of Things & SIC & Successive Interference Cancellation \\
\hline IRS & Intelligent Reflecting Surface & SNR & Signal to Noise Ratio \\
\hline ISL & Inter-satellite Link & SS & Spread Spectrum \\
\hline ITU & International Telecommunications Union & SOP & Secrecy Outage Probability \\
\hline $\mathrm{kg}$ & kilogram & SU & Secondary User \\
\hline $\mathrm{km}$ & kilometer & $\mathrm{THz}$ & Terahertz \\
\hline LEO & Low Earth Orbit & TMR & Triple Module Redundancy \\
\hline LFDA & Linear Frequency Diverse Array & UAV & Unmanned Aerial Vehicle \\
\hline LMS & Least Mean Square & & \\
\hline
\end{tabular}

the stylized Fig. 25. Below we briefly touch upon the most influential design factors:

- It is of vital importance to determine the specific choice of security countermeasures employed by the different subsystems of LEO SCSs according to the specific tradeoff between the security improvement attained and its cost in terms of the overhead imposed. Explicitly, traditional mathematics-based encryption relying on excessive computational complexity is not suitable for the powerlimited space-borne payloads, but it is routinely used at the ground facilities for improving the security level;

- Advanced security-oriented antennas are capable of substantially mitigating the eavesdropping probability. However, the family of NOMA-based TPC techniques has to strike a trade-off between the capacity and the security level attained;

- Interference coordination techniques - including power control, cognitive radio, and so on - are routinely adopted for mitigating the CCI between terrestrial systems and GEO SCSs. These techniques have to be jointly optimized in conjunction with GEO SCSs or terrestrial systems;

- SS techniques exhibit natural anti-eavesdropping and anti-jamming capabilities. Hence they constitute the preferred choice of waveforms for safeguarding the secu- rity of LEO SCSs. However, they reduce the effective throughput by a factor commensurate with the spreading factor. Additionally, there is also a trade-off between the integrity and the security of MC-DSSS systems, as seen in Fig. 18. A fraction of the sub-carriers may be hidden in the interference, which improves the confidentiality of the transmitted signal, but potentially degrades the BER;

- Some countermeasures such as advanced securityoriented antennas, as well as both transform and temporal domain adaptive filtering, can also be used for improved jamming mitigation. Among them, the low complexity LMS algorithm - which is a popular design option of temporal domain adaptive filtering technique - is eminently suitable for space-borne payloads. However, the selection of $\mu$ affects the trade-off between the security and the latency as shown in Fig. 19;

- There is no doubt that TMR mitigates the impact of SEU, but at the cost of a certain additional resource consumption. Periodical refreshing is another protection measure, which may be beneficially combined with the TMR technique for improving the FPGAs' reliability.

\section{REFERENCES}

[1] S. Liu, Z. Gao, Y. Wu et al., "LEO satellite constellations for 5G and beyond: How will they reshape vertical domains?" IEEE Commun. 
Mag., vol. 59, no. 7, pp. 30-36, Jul. 2021.

[2] K. An, M. Lin, J. Ouyang et al., "Secure transmission in cognitive satellite terrestrial networks," IEEE J. Sel. Areas Commun., vol. 34 no. 11, pp. 3025-3037, Nov. 2016.

[3] E. Meng and $\mathrm{X}$. Bu, "Two-dimensional joint acquisition of Doppler factor and delay for MC-DS-CDMA in LEO satellite system," IEEE Access, vol. 8, pp. 148 203-148 213, Aug. 2020.

[4] B. Di, L. Song, Y. Li et al., "Ultra-dense LEO: Integration of satellite access networks into 5G and beyond," IEEE Wireless Commun., vol. 26, no. 2, pp. 62-69, Apr. 2019

[5] I. Leyva-Mayorga, B. Soret, M. Röper et al., "LEO small-satellite constellations for $5 \mathrm{G}$ and beyond-5G communications," IEEE Access, vol. 8, pp. $184955-184964$, Oct. 2020

[6] S. R. Pratt, R. A. Raines, C. E. Fossa et al., "An operational and performance overview of the Iridium low earth orbit satellite system," IEEE Commun. Surveys Tuts., vol. 2, no. 2, pp. 2-10, Second Quart. 1999.

[7] F. J. Dietrich, P. Metzen, and P. Monte, "The Globalstar cellular satellite system," IEEE Trans. Antennas Propagat., vol. 46, no. 6, pp. 935-942, Jun. 1998.

[8] P. Wang, J. Zhang, X. Zhang et al., "Convergence of satellite and terrestrial networks: A comprehensive survey," IEEE Access, vol. 8, pp. 5550-5588, Dec. 2019.

[9] R. Cochetti, Low Earth Orbit (LEO) Mobile Satellite Communications Systems. Wiley, Oct. 2014, pp. 119-156.

[10] H. Boiardt and C. Rodriguez, "Low earth orbit nanosatellite communications using Iridium's network," IEEE Aerosp. Electron. Syst. Mag., vol. 25 , no. 9 , pp. 35-39, Sep. 2010.

[11] P. Timothy and J. Allnutt, Satellite communications. John Wiley \& Sons, Oct. 2019

[12] S. Xia, Q. Jiang, C. Zou et al., "Beam coverage comparison of LEO satellite systems based on user diversification," IEEE Access, vol. 7, pp. $181656-181667$, Dec. 2019.

[13] L. Perino-Gallice, O. Masson, M. Bel et al., "Batteries for satellites constellation, using lean manufacturing for space industry," in Proc. European Space Power Conference, Juan-les-Pins, France, Dec. 2019, pp. 1-6.

[14] Y. Liu, H. Xing, C. Pan et al., "Multiple-antenna-assisted nonorthogonal multiple access," IEEE Wireless Commun., vol. 25, no. 2, pp. 17-23, Apr. 2018.

[15] T. Duan and V. Dinavahi, "Starlink space network-enhanced cyber-physical power system," IEEE Trans. Smart. Grid., vol. 12, no. 4, pp. 3673-3675, Mar. 2021.

[16] X. Zhu, Y. Yang, z. liu et al., "Towards 6G wireless communication networks: Vision, enabling technologies, and new paradigm shifts," Sci. China Inf. Sci., pp. 1-74, Nov. 2020.

[17] C. Fossa, R. Raines, G. Gunsch et al., "An overview of the Iridium (R) low earth orbit (LEO) satellite system," in Proc. IEEE National Aerospace and Electronics Conference, Dayton, OH, USA, Jul. 1998, pp. $152-159$.

[18] W. Zhang, M. Yang, J. Yang et al., Low Earth Orbit High-Throughput Satellite Multibeam Design Schemes. Communications, Signal Processing, and Systems, May 2019.

[19] Y. Henri, The OneWeb Satellite System. Cham: Springer International Publishing, Feb. 2020, pp. 1-10.

[20] I. D. Portillo, B. G. Cameron, and E. F. Crawley, "A technical comparison of three low earth orbit satellite constellation systems to provide global broadband," Acta Astronaut., vol. 159, pp. 123-135, Jun. 2019.

[21] K. Ravel, C. Koechlin, E. Prevost et al., "Optical switch matrix development for new concepts of photonic based flexible telecom payloads," in Proc. International Conference on Space Optics, vol. 11180, Chania, Greece, Oct. 2018, pp. 1319-1332.

[22] R. Jewett. Telesat Picks Thales for Lightspeed LEO Constellation. (Feb. 2, 2021). [Online]. Available: https://www.satellitetoday.com/ broadband/2021/02/09/

[23] C. Henry. Kepler decides to build its 140-satellite cubesat constellation in-house. (Jan. 29, 2020). [Online]. Available: https://spacenews.com/ kepler-decides-to-build-its-140-satellite

[24] P. Butani. GEO-HTS is here today but... Is LEOHTS the future? (Jan. 20, 2015). [Online]. Available: http://satcompost.com/geo-hts-is-here-today-but-is-leo-hts-the-future/

[25] O. B. Osoro and E. J. Oughton, "A techno-economic framework for satellite networks applied to low earth orbit constellations: Assessing Starlink, OneWeb and Kuiper," IEEE Access, vol. 9, pp. 141611 141625 , Oct. 2021.
[26] P. Paganini. Hacking the Iridium network could be very easy. (Aug 23, 2015). [Online]. Available: https://securityaffairs.co/wordpress/ 39510/hacking/hacking-iridium-network

[27] M. RIZK. Network: Signal jammed in Egypt during comedy show. (Mar. 9, 2014). [Online]. Available: http://www.citicsat.com/Info_ News/14/125

[28] L. H. Newman. Hackers are building an army of cheap satellite trackers. (Aug. 04, 2020). [Online]. Available: https://www.wired.com/ story/nyansat-open-source-satellite-tracker/

[29] E. Nakashima. Russian hacker group exploits satellites to steal data, hide tracks. (Sep. 9, 2015). [Online]. Available: https://www.washingtonpost.com/world/national-security/ russian-hacker-group-exploits-satellites-to-steal-data-hide-tracks

[30] AEDT. Hackers could shut down satellites - or turn them into weapons. (Feb. 13, 2020). [Online]. Available: https://theconversation.com/ hackers-could-shut-down-satellites-or-turn-them-into-weapons- 130932

[31] M. Tafazoli, "A study of on-orbit spacecraft failures," Acta Astronaut., vol. 64, no. 2-3, pp. 195-205, Oct. 2008.

[32] B. Li, J. Huang, Y. Feng et al., "A machine learning-based approach for improved orbit predictions of LEO space debris with sparse tracking data from a single station," IEEE Trans. Aerosp. Electron. Syst., vol. 56, no. 6, pp. 4253-4268, Apr. 2020.

[33] Q. Chen, W. Meng, S. Han et al., "Service-oriented fair resource allocation and auction for civil aircrafts augmented space-air-ground integrated networks," IEEE Trans. Veh. Technol., vol. 69, no. 11, pp. 13658-13672, Sep. 2020.

[34] M. G. Padmashree, J. S. Arunalatha, and K. R. Venugopal, "HPAKE: Hybrid precocious authentication and key establishment in IoT," in Proc. Car. C. Secur., Chennai, India, Oct. 2019, pp. 1-6.

[35] R. S. M. Joshitta and L. Arockiam, "Device authentication mechanism for IoT enabled healthcare system," in Proc. International Conference on Algorithms, Methodology, Models and Applications in Emerging Technologies, Chennai, India, Feb. 2017, pp. 1-6.

[36] I. Ali, N. Al-Dhahir, and J. E. Hershey, "Doppler characterization for LEO satellites," IEEE Trans. Commun., vol. 46, no. 3, pp. 309-313, Mar. 1998.

[37] D. Valentini, A. Pasini, G. Pace et al., "Green propellant thruster design for LEO platforms active debris removal," in Proc. ESA Space Propulsion 2018 Conference, Seville, Spain, May. 2018, pp. 1-11.

[38] Z. Lin, M. Lin, J. Ouyang et al., "Robust secure beamforming for multibeam satellite communication systems," IEEE Trans. Veh. Technol., vol. 68, no. 6, pp. 6202-6206, Apr. 2019.

[39] K. Guo, K. An, B. Zhang et al., "Physical layer security for multiuser satellite communication systems with threshold-based scheduling scheme," IEEE Trans. Veh. Technol., vol. 69, no. 5, pp. 5129-5141, May 2020

[40] S. Xu, J. Liu, Y. Cao, J. Li et al., "Intelligent reflecting surface enabled secure cooperative transmission for satellite-terrestrial integrated networks," IEEE Trans. Veh. Technol., vol. 70, no. 2, pp. 2007-2011, Feb. 2021

[41] M. Yi, X. Xu, and L. Xu, "An intelligent communication warning vulnerability detection algorithm based on IoT technology," IEEE Access, vol. 7, pp. 164 803-164 814, Nov. 2019.

[42] K. Vieira, F. L. Koch, J. M. Sobral et al., "Autonomic intrusion detection and response using Big Data," IEEE Syst. J., vol. 14, no. 2, pp. 1984-1991, Jun. 2020.

[43] G. He, W. Su, S. Gao et al., "Roachain: Securing route origin authorization with blockchain for inter-domain routing," IEEE Trans. Netw. Service Manag., vol. 18, no. 2, pp. 1690-1705, 2021.

[44] N. Wang and J. Li, "Shortest path routing with risk control for compromised wireless sensor networks," IEEE Access, vol. 7, pp. 19303-19311, Feb. 2019.

[45] Y. Chen, L. Wang, and S. Wang, "Stochastic blockchain for IoT data integrity," IEEE Trans. Netw. Sci. Eng., vol. 7, no. 1, pp. 373-384, Mar. 2020.

[46] J. Pacheco, V. H. Benitez, L. C. Félix-Herrán et al., "Artificial neural networks-based intrusion detection system for Internet of Things fog nodes," IEEE Access, vol. 8, pp. 73 907-73918, May 2020.

[47] M. Mohammad, B. Cambou, C. R. Philabaum et al., "Resilient password manager using physical unclonable functions," IEEE Access, vol. 9, pp. $17060-17070$, Jan. 2021.

[48] Y. Su, Y. Liu, Y. Zhou et al., "Broadband LEO satellite communications: Architectures and key technologies," IEEE Wireless Commun., vol. 26, no. 2, pp. 55-61, Apr. 2019.

[49] E. Re, A. Murrell, and D. Roques, "Radio resource management for large constellations in a spectrum sharing environment," Int. J. Satell. Commun. Netw., vol. 39, no. 1, pp. 78-91, Oct. 2020. 
[50] H. Wang, R. Ren, D. Qu et al., "A radio environment mapping based spectrum awareness for cognitive space information network with GEO and LEO coexistence," in Proc. International Conference on Wireless Communications and Signal Processing, Nanjing, China, Dec. 2020, pp. 654-659.

[51] R. Ge, D. Bian, J. Cheng et al., "Joint user pairing and power allocation for NOMA-Based GEO and LEO satellite network," IEEE Access, vol. 9, pp. 93 255-93 266, May 2021.

[52] Y. Liao, X. Wu, Z. Wu et al., "Robust constrained inverse beamforming algorithm based on space time adaptive processing," IEEE Access, vol. 7, pp. 55 191-55 198, May 2019.

[53] C. Han, L. Huo, X. Tong et al., "Spatial anti-jamming scheme for Internet of Satellites based on the deep reinforcement learning and stackelberg game," IEEE Trans. Veh. Technol., vol. 69, no. 5, pp. 53315342, May 2020

[54] H. Yang, Z. Xiong, J. Zhao et al., "Intelligent reflecting surface assisted anti-jamming communications: A fast reinforcement learning approach," IEEE Trans. Wireless Commun., vol. 20, no. 3, pp. 19631974, Mar. 2021

[55] V. Weerackody, "Satellite diversity to mitigate jamming in LEO satellite mega-constellations," in Proc. IEEE Int. Conf. Commun. China workshops, Montreal, QC, Canada, Jun. 2021, pp. 1-6.

[56] C. A. Rigo, M. Luza, L, E. D. Tramontin et al., "A fault-tolerant reconfigurable platform for communication modules of satellites," in Proc. Latin American Test Symposium, Santiago, Chile, May 2019, pp. $1-6$.

[57] R. M. Monreal, J. Alvarez, G. Dennis et al., "Impact of single event effects on key electronic components for COTS-based satellite systems," in Proc. Radiation Effects Data Workshop, San Antonio, TX, USA, Jul. 2019, pp. 1-7.

[58] C. Gkiokas and M. Schoeberl, "A fault-tolerant time-predictable processor," in Proc. Nordic Circuits and Systems Conference, Helsinki, Finland, Oct. 2019, pp. 1-6.

[59] Z. Gao, L. Yan, J. Zhu et al., "Radiation tolerant Viterbi decoders for on-board processing (OBP) in satellite communications," China Commun., vol. 17, no. 1, pp. 140-150, Jan. 2020.

[60] Z. Gao, L. Zhang, R. Han et al., "Reliability evaluation of Turbo decoders implemented on SRAM-FPGAs," in Proc. VLSI Test Symposium, San Diego, CA, USA, Jun. 2020, pp. 1-6.

[61] K. Saito, S. Hatta, and T. Hanada, "Digital currency design for sustainable active debris removal in space," IEEE Trans. Comput. Soc. Syst., vol. 6, no. 1, pp. 127-134, Jan. 2019.

[62] B. Wei and B. D. Nener, "Multi-sensor space debris tracking for space situational awareness with labeled random finite sets," IEEE Access, vol. 7, pp. 36991-37 003, Mar. 2019.

[63] J. Yang, X. Hou, Y. H. Hu et al., "A reinforcement learning scheme for active multi-debris removal mission planning with modified upper confidence bound tree search," IEEE Access, vol. 8, pp. 108461 108 473, Jun. 2020.

[64] D. Cataldo, L. Gentile, S. Ghio et al., "Multibistatic radar for space surveillance and tracking," IEEE Aerosp. Electron. Syst. Mag., vol. 35, no. 8, pp. 14-30, Aug. 2020.

[65] Y. Zou, J. Zhu, L. Yang et al., "Securing physical-layer communications for cognitive radio networks," IEEE Commun. Mag., vol. 53, no. 9, pp. 48-54, Sep. 2015

[66] Y. Zou, J. Zhu, X. Wang et al., "A survey on wireless security: Technical challenges, recent advances, and future trends," Proc. IEEE, vol. 104, no. 9, pp. 1727-1765, Sep. 2016.

[67] J. Liu, Y. Shi, Z. M. Fadlullah et al., "Space-air-ground integrated network: A survey," IEEE Commun. Surveys Tuts., vol. 20, no. 4, pp. 2714-2741, Fourth Quart. 2018.

[68] A. Fotouhi, H. Qiang, M. Ding et al., "Survey on UAV cellular communications: Practical aspects, standardization advancements, regulation, and security challenges," IEEE Commun. Surveys Tuts., vol. 21, no. 4, pp. 3417-3442, Fourth Quart. 2019.

[69] M. Lin, Q. Huang, T. de Cola et al., "Integrated 5G-satellite networks: A perspective on physical layer reliability and security," IEEE Wireless Commun., vol. 27, no. 6, pp. 152-159, Dec. 2020.

[70] O. Kodheli, E. Lagunas, N. Maturo et al., "Satellite communications in the new space era: A survey and future challenges," IEEE Commun. Surveys Tuts., vol. 23, no. 1, pp. 70-109, First Quart. 2021.

[71] A. Coronetti, R. G. Alía, J. Budroweit et al., "Radiation hardness assurance through system-level testing: Risk acceptance, facility requirements, test methodology, and data exploitation," IEEE Trans. Nucl. Sci., vol. 68, no. 5, pp. 958-969, May 2021.
[72] H. Guo, J. Li, J. Liu et al., "A survey on space-air-ground-sea integrated network security in 6G," IEEE Commun. Surveys Tuts., Fourth Quart. 2021. [Online]. Available: 10.1109/COMST.2021.3131332

[73] Y. Zou, J. Zhu, X. Li et al., "Relay selection for wireless communications against eavesdropping: A security-reliability trade-off perspective," IEEE Netw., vol. 30, no. 5, pp. 74-79, Sep. 2016.

[74] F. Shu, T. Shen, L. Xu et al., "Directional modulation: A physical-layer security solution to B5G and future wireless networks," IEEE Netw. vol. 34, no. 2, pp. 210-216, Apr. 2020.

[75] S. Yan, X. Wang, Z. Li et al., "Cooperative jamming for physical layer security in hybrid satellite terrestrial relay networks," China Commun., vol. 16, no. 12, pp. 154-164, Dec. 2019.

[76] T. Li, J. Ye, J. Dai et al., "Secure UAV-to-vehicle communications," IEEE Trans. Commun., vol. 69, no. 8, pp. 5381-5393, Apr. 2021.

[77] X. Zhu, C. Jiang, L. Kuang et al., "Non-orthogonal multiple access based integrated terrestrial-satellite networks," IEEE J. Sel. Areas Commun., vol. 35, no. 10, pp. 2253-2267, Oct. 2017.

[78] J. Pavur, D. Moser, V. Lenders et al., "Secrets in the sky: On privacy and infrastructure security in DVB-S satellite broadband," in Proc. Conference on Security and Privacy in Wireless and Mobile Networks, Miami, FL, USA, May 2019, pp. 277-284.

[79] CITIC. How to Effectively Reduce the Impact of Ground Interference on Satellite transponder? (Mar. 18, 2019). [Online]. Available: http://www.citicsat.com/Info_News/14/125

[80] H. Choi and H. Moon, "Blind estimation of spreading sequence and data bits in direct-sequence spread spectrum communication systems," IEEE Access, vol. 8, pp. $148066-148$ 074, Aug. 2020.

[81] A. Roy-Chowdhury, J. S. Baras, M. Hadjitheodosiou et al., "Security issues in hybrid networks with a satellite component," IEEE Wireless Commun., vol. 12, no. 6, pp. 50-61, Dec. 2005.

[82] G. Cluley. Could this be the world's most harmless IoT botnet? (May 08, 2020). [Online]. Available: https://www.bitdefender.com/ blog/hotforsecurity/worlds-harmless-iot-botnet

[83] E. Shi and A. Perrig, "Designing secure sensor networks," IEEE Wireless Commun., vol. 11, no. 6, pp. 38-43, Dec. 2004.

[84] X. Chen, K. Makki, K. Yen et al., "Sensor network security: A survey," IEEE Commun. Surveys Tuts., vol. 11, no. 2, pp. 52-73, Second Quart. 2009.

[85] R. Radhakrishnan, W. W. Edmonson, F. Afghah et al., "Survey of intersatellite communication for small satellite systems: Physical layer to network layer view," IEEE Commun. Surveys Tuts., vol. 18, no. 4, pp. 2442-2473, Fourth Quart. 2016.

[86] H. Chen, Y. Xiao, J. Li et al., "The OCC-CDMA/OS for 4G wireless," IEEE Veh. Technol. Mag., vol. 1, no. 3, pp. 12-21, Sep. 2006.

[87] H. Chen, D. Hank, M. E. Maganaz et al., "Design of next-generation CDMA using orthogonal complementary codes and offset stacked spreading," IEEE Wireless Commun., vol. 14, no. 3, pp. 61-69, Jul. 2007.

[88] J. Li, A. Huang, M. Guizani et al., "Inter-group complementary codes for interference-resistant CDMA wireless communications," IEEE Trans. Wireless Commun., vol. 7, no. 1, pp. 166-174, Jan. 2008.

[89] J. P. Choi and C. Joo, "Challenges for efficient and seamless spaceterrestrial heterogeneous networks," IEEE Commun. Mag., vol. 53, no. 5, pp. 156-162, May 2015.

[90] J. Ye, G. Pan, and M. S. Alouini, "Earth rotation-aware non-stationary satellite communication systems: Modeling and analysis," IEEE Trans. Wireless Commun., vol. 20, no. 9, pp. 5942-5956, Apr. 2021.

[91] S. Chen, Y. C. Liang, S. Sun et al., "Vision, requirements, and technology trend of 6G: How to tackle the challenges of system coverage, capacity, user data-rate and movement speed," IEEE Wireless Commun., vol. 27, no. 2, pp. 218-228, Apr. 2020.

[92] D. He, S. Chan, and M. Guizani, "Communication security of unmanned aerial vehicles," IEEE Wireless Commun., vol. 24, no. 4, pp. 134-139, Aug. 2017.

[93] J. Magiera and R. Katulski, "Detection and mitigation of GPS spoofing based on antenna array processing," J. Appl. Res. Technol., vol. 13, no. 1 , pp. 45-57, Feb. 2015.

[94] A. S. Abdalla, K. Powell, V. Marojevic et al., "UAV-assisted attack prevention, detection, and recovery of $5 \mathrm{G}$ networks," IEEE Wireless Commun., vol. 27, no. 4, pp. 40-47, Aug. 2020.

[95] Y. Tian, G. Pan, M. A. Kishk et al., "Stochastic analysis of cooperative satellite-UAV communications," IEEE Trans. Wireless Commun., Oct. 2021. [Online]. Available: 10.1109/TWC.2021.3121299

[96] K. Wang, H. Lei, G. Pan et al., "Detection performance to spatially random UAV using the ground vehicle," IEEE Trans. Veh. Technol., vol. 69 , no. 12 , pp. 16320-16324, Dec. 2020. 
[97] H. Cao, L. Wu, Y. Chen et al., "Analysis on the security of satellite internet," in China Cyber Security Annual Conference, Beijing, China, Dec. 2020, pp. 193-205.

[98] K. Mayyas, "Performance analysis of the deficient length LMS adaptive algorithm," IEEE Trans. Signal Process., vol. 53, no. 8, pp. 2727-2734, Aug. 2005.

[99] R. Merched and A. Sayed, "An embedding approach to frequencydomain and subband adaptive filtering," IEEE Trans. Signal Process., vol. 48, no. 9, pp. 2607-2619, 2000.

[100] V. Vargas, P. Ramos, R. Velazco et al., "Evaluating SEU fault-injection on parallel applications implemented on multicore processors," in Proc. Latin American Symposium on Circuits Systems, Montevideo, Uruguay, Feb. 2015, pp. 1-4.

[101] Union of Concerned Scientists. UCS Satellite Database. (Sep. 1, 2021). [Online]. Available: https://www.ucsusa.org/resources/ satellite-database

[102] L. David. Effects of worst satellite breakups in history still felt today. (Jan. 28, 2013). [Online]. Available: https://www.space.com/ 19450-space-junk-worst-events-anniversaries.html

[103] J. Foust. ESA spacecraft dodges potential collision with starlink satellite. (Sep. 2, 2019). [Online]. Available: https://spacenews.com/ esa-spacecraft-dodges-potential-collision-with-starlink-satellite/

[104] Accessed: Dec. 28, 2021. [Online]. Available: https://www.unoosa.org/ oosa/en/oosadoc/data/documents/2021/aac.105/aac.1051262_0.html

[105] Accessed: Dec. 28, 2021. [Online]. Available: https://www.esa.int/ Safety_Security/Space_Debris/Space_debris_by_the_numbers

[106] J. Drmola and T. Hubik, "Kessler syndrome: System dynamics model," Space Policy, vol. 44-45, pp. 29-39, Aug. 2018.

[107] Accessed: Dec. 28, 2021. [Online]. Available: https: //www.esa.int/ESA_Multimedia/Images/2009/05/ESA_built-solar_ cells_retrieved_from_the_Hubble_Space_Telescope_in_2002

[108] D. M. Lear. STS-118 Radiator Impact Damage. (Jan. 1, 2008). [Online]. Available: https://ntrs.nasa.gov/citations/20080010742

[109] L. David. Copernicus Sentinel-1A satellite hit by space particle. (Aug. 31, 2016). [Online]. Available: https://www.space.com/ 33920-european-satellite-space-particle-strike.html

[110] E. Howell. Space station robotic arm hit by orbital debris in 'lucky strike'. (May 31, 2021). [Online]. Available: https: //www.space.com/space-station-robot-arm-orbital-debris-strike

[111] Space debris and human spacecraft. (May 26, 2021). [Online]. Available: https://www.nasa.gov/mission_pages/station/news/orbital_ debris.html

[112] S. Goel and R. Negi, "Guaranteeing secrecy using artificial noise," Trans. Wireless Commun., vol. 7, no. 6, pp. 2180-2189, Jun. 2008.

[113] X. Ding, T. Song, Y. Zou et al., "Security-reliability tradeoff analysis of artificial noise aided two-way opportunistic relay selection," IEEE Trans. Veh. Technol., vol. 66, no. 5, pp. 3930-3941, May 2017.

[114] Y. Deng, L. Wang, S. A. R. Zaidi et al., "Artificial-noise aided secure transmission in large scale spectrum sharing networks," IEEE Trans. Commun., vol. 64, no. 5, pp. 2116-2129, May 2016.

[115] S. Yun, J.-M. Kang, I.-M. Kim et al., "Deep artificial noise: Deep learning-based precoding optimization for artificial noise scheme," IEEE Trans. Veh. Technol., vol. 69, no. 3, pp. 3465-3469, Mar. 2020.

[116] Y. Liao, J. Wang, and Q. H. Liu, "Transmit beampattern synthesis for frequency diverse array with particle swarm frequency offset optimization," IEEE Trans. Antennas Propag., vol. 69, no. 2, pp. 892 901, Feb. 2021.

[117] W. Wang, "Frequency diverse array antenna: New opportunities," IEEE Antennas Propagat. Mag., vol. 57, no. 2, pp. 145-152, Apr. 2015.

[118] J. Hu, S. Yan, F. Shu et al., "Artificial-noise-aided secure transmission with directional modulation based on random frequency diverse arrays,' IEEE Access, vol. 5, pp. 1658-1667, Jan. 2017.

[119] Y. Xu, X. Shi, W. Li et al., "Low-sidelobe range-angle beamforming with FDA using multiple parameter optimization," IEEE Trans. Aerosp. Electron. Syst., vol. 55, no. 5, pp. 2214-2225, Oct. 2019.

[120] L. Fan, N. Yang, T. Q. Duong et al., "Exploiting direct links for physical layer security in multiuser multirelay networks," IEEE Trans. Wireless Commun., vol. 15, no. 6, pp. 3856-3867, Jun. 2016.

[121] L. Wang, Y. Cai, Y. Zou et al., "Joint relay and jammer selection improves the physical layer security in the face of CSI feedback delays," IEEE Trans. Veh. Technol., vol. 65, no. 8, pp. 6259-6274, Aug. 2016.

[122] V. Bankey and P. K. Upadhyay, "Physical layer security of multiuser multirelay hybrid satellite-terrestrial relay networks," IEEE Trans. Veh. Technol., vol. 68, no. 3, pp. 2488-2501, Mar. 2019.
[123] J. Zhang, E. Björnson, M. Matthaiou et al., "Prospective multiple antenna technologies for beyond 5G," IEEE J. Sel. Areas Commun., vol. 38, no. 8, pp. 1637-1660, Aug. 2020.

[124] A. I. Perez-Neira, M. A. Vazquez, M. R. B. Shankar et al., "Signal processing for high-throughput satellites: Challenges in new interferencelimited scenarios," IEEE Signal Process. Mag., vol. 36, no. 4, pp. 112131, Jul. 2019.

[125] L. You, K. X. Li, J. Wang et al., "Massive MIMO transmission for LEO satellite communications," IEEE J. Sel. Areas Commun., vol. 38 , no. 8 , pp. 1851-1865, Aug. 2020

[126] J. Chu, X. Chen, C. Zhong et al., "Robust design for NOMA-based multibeam LEO satellite Internet of Things," IEEE Internet Things $J$., vol. 8, no. 3, pp. 1959-1970, Feb. 2021.

[127] P. Rocca, R. L. Haupt, and A. Massa, "Interference suppression in uniform linear arrays through a dynamic thinning strategy," IEEE Trans. Antennas Propagat., vol. 59, no. 12, pp. 4525-4533, Dec. 2011.

[128] Tapan K. S., Hong W., Sheeyun P. et al., "A deterministic leastsquares approach to space-time adaptive processing (STAP)," IEEE Trans. Antennas Propagat., vol. 49, no. 1, pp. 91-103, Jan. 2001.

[129] D. Cristallini and W. Burger, "A robust direct data domain approach for STAP," IEEE Trans. Signal Process., vol. 60, no. 3, pp. 1283-1294, Mar. 2012

[130] T. Ikuma and A. A. Beex, "Improved mean-square error estimate for the LMS transversal equalizer with narrowband interference," IEEE Trans. Signal Process., vol. 56, no. 10, pp. 5273-5277, Oct. 2008.

[131] J. J. Perez-Solano, S. Felici-Castell, and M. A. Rodriguez-Hernandez, "Narrowband interference suppression in frequency-hopping spread spectrum using undecimated wavelet packet transform," IEEE Trans. Veh. Technol., vol. 57, no. 3, pp. 1620-1629, May 2008

[132] E. Lagunas, S. K. Sharma, S. Maleki et al., "Power control for satellite uplink and terrestrial fixed-service co-existence in Ka-band," in Proc. Vehicular Technology Conference, Boston, MA, USA, Sep. 2015, pp. $1-5$.

[133] E. Lagunas, S. Maleki, S. Chatzinotas et al., "Power and rate allocation in cognitive satellite uplink networks," in Proc. IEEE Int. Conf. Comm., Kuala Lumpur, Malaysia, May 2016, pp. 1-6.

[134] R. Li, P. Gu, and C. Hua, "Optimal beam power control for co-existing multibeam GEO and LEO satellite system," in Proc. International Conference on Wireless Communications and Signal Processing, Xi' an, China, Oct. 2019, pp. 1-6.

[135] M. Jia, Z. Li, X. Gu, and Q. Guo, "Joint multi-beam power control for LEO and GEO spectrum-sharing networks," in Proc. IEEE Int. Conf. Comm., Xiamen, China, Jul. 2021, pp. 841-846.

[136] H. Wang, C. Wang, J. Yuan et al., "Coexistence downlink interference analysis between LEO system and GEO system in Ka band," in Proc. IEEE Int. Conf. Comm., Beijing, China, Aug. 2018, pp. 465-469.

[137] C. Zhang, J. Jin, H. Zhang et al., "Spectral coexistence between LEO and GEO satellites by optimizing direction normal of phased array antennas," China Commun., vol. 15, no. 6, pp. 18-27, Jun. 2018.

[138] T. Li, J. Jin, W. Li et al., "Research on interference avoidance effect of OneWeb satellite constellation's progressive pitch strategy," Int. J. Satell. Commun. Netw., Mar. 2021.

[139] G. Ding, Y. Jiao, J. Wang et al., "Spectrum inference in cognitive radio networks: Algorithms and applications," IEEE Commun. Surveys Tuts., vol. 20, no. 1, pp. 150-182, First Quart. 2018.

[140] W. Liang, S. X. Ng, and L. Hanzo, "Cooperative overlay spectrum access in cognitive radio networks," IEEE Commun. Surveys Tuts. vol. 19, no. 3, pp. 1924-1944, Third Quart. 2017.

[141] C. Jiang, Y. Chen, K. J. R. Liu et al., "Renewal-theoretical dynamic spectrum access in cognitive radio network with unknown primary behavior," IEEE J. Sel. Areas Commun., vol. 31, no. 3, pp. 406-416, Mar. 2013

[142] T. Yucek and H. Arslan, "A survey of spectrum sensing algorithms for cognitive radio applications," IEEE Commun. Surveys Tuts., vol. 11, no. 1, pp. 116-130, First Quart. 2009.

[143] C. Zhang, C. Jiang, J. Jin et al., "Spectrum sensing and recognition in satellite systems," IEEE Trans. Veh. Technol., vol. 68, no. 3, pp. 2502-2516, Mar. 2019.

[144] P. Gu, R. Li, C. Hua et al., "Dynamic cooperative spectrum sharing in a multi-beam LEO-GEO co-existing satellite system," IEEE Trans. Wireless Commun., Aug. 2021. [Online]. Available: 10.1109/TWC.2021.3102704

[145] J. Hu, G. Li, D. Bian et al., "Energy-efficient cooperative spectrum sensing in cognitive satellite terrestrial networks," IEEE Access, vol. 8 , pp. $161396-161405$, Sep. 2020. 
[146] Y. Wang, X. Ding, and G. Zhang, "A novel dynamic spectrum-sharing method for GEO and LEO satellite networks," IEEE Access, vol. 8, pp. 147 895-147906, Aug. 2020.

[147] J. Tang, D. Bian, G. Li et al., "Resource allocation for LEO beamhopping satellites in a spectrum sharing scenario," IEEE Access, vol. 9, pp. 56468-56478, Apr. 2021.

[148] X. Ding, L. Feng, Y. Zou et al., "Deep learning aided spectrum prediction for satellite communication systems," IEEE Trans. Veh. Technol., vol. 69, no. 12, pp. 16314-16319, Dec 2020.

[149] C. Yang, Q. Zhang, Q. Tian et al., "In-line interference mitigation method based on adaptive modulation and coding for satellite system," in Proc. International Conference on Optical Communications and Networks, Wuzhen, China, Aug. 2017, pp. 1-3.

[150] T. Pultarova. The world's first wooden satellite will launch this year. (Jun. 15, 2021). [Online]. Available: https://www.space.com/ first-wooden-satellite-will-launch-in-2021

[151] R. Dudziak, S. Tuttle, and S. Barraclough, "Harpoon technology development for the active removal of space debris," Adv. Space Res., vol. 56, no. 3, pp. 509-527, Aug. 2015.

[152] T. Pultarova, "Robots, harpoons and nets: How to clean up orbital rubbish," Engineering Technology, vol. 13, no. 10, pp. 62-65, Nov. 2018.

[153] B. Yang, "Research on the strategy how to clean up space debris," in Proc. International Conference on Education, Management and Computing Technology, Hangzhou, China, Apr. 2016, pp. 1054-1057.

[154] S. Nishida, S. Kawamoto, Y. Okawa et al., "Space debris removal system using a small satellite," Acta Astronaut., vol. 65, no. 1, pp. 95-102, Aug. 2009.

[155] D. Shiga. Giant balloons could clear out space junk. (Aug. 4, 2010). [Online]. Available: https://www.newscientist.com/article/ dn19262-giant-balloons-could-clear-out-space-junk/

[156] B. Ren, "The most optimal device for removing space debris," in Proc. International Conference on Machinery, Materials, Environment, Biotechnology and Computer, Tianjin, China, Jun. 2016, pp. 1144 1147.

[157] H. Yao, L. Wang, X. Wang et al., "The space-terrestrial integrated network: An overview," IEEE Commun. Mag., vol. 56, no. 9, pp. 178185, Sep. 2018.

[158] G. Baldini, T. Sturman, A. R. Biswas et al., "Security aspects in software defined radio and cognitive radio networks: A survey and a way ahead," IEEE Commun. Surveys Tuts., vol. 14, no. 2, pp. 355-379, Second Quart. 2012.

[159] C. Javali, G. Revadigar, M. Ding et al., "Secret key generation by virtual link estimation," in Proc. ACM BodyNets, Sydney, Australia, Sep. 2015.

[160] R. Scholtz, "The origins of spread-spectrum communications," IEEE Trans. Commun., vol. 30, no. 5, pp. 822-854, May 1982.

[161] L. Hanzo, L.-L. Yang, E. Kuan et al., Single-and multi-carrier DS CDMA: Multi-user detection, space-time spreading, synchronisation, standards and networking. John Wiley \& Sons, 2003.

[162] R. Iltis and L. Milstein, "Performance analysis of narrow-band interference rejection techniques in DS spread-spectrum systems," IEEE Trans. Commun., vol. 32, no. 11, pp. 1169-1177, Nov. 1984.

[163] M. K. Simon and A. Polydoros, "Coherent detection of frequencyhopped quadrature modulations in the presence of jamming," IEEE Trans. Commun., vol. 29, no. 11, pp. 1644-1660, Nov. 1981.

[164] G. Li, Q. Wang, V. K. Bhargava et al., "Maximum-likelihood diversity combining in partial-band noise," IEEE Trans. Commun., vol. 46, no. 12, pp. 1569-1574, Dec. 1998.

[165] J. Kang and K. Teh, "Performance analyses of coherent fast frequencyhopping spread-spectrum systems with partial band noise jamming and AWGN," in Proc. Fourth International Conference on Information, Communications and Signal Processing, 2003 and the Fourth Pacific Rim Conference on Multimedia, vol. 1, Singapore, Dec. 2003, pp. 678681.

[166] S. Wang, S. Chen, A. Wang et al., "Joint timing and channel estimation for bandlimited long-code-based MC-DS-CDMA: A low-complexity near-optimal algorithm and the CRLB," IEEE Trans. Commun., vol. 61, no. 5, pp. 1998-2011, May 2013.

[167] S. C. Douglas, Quanhong Zhu, and K. F. Smith, "A pipelined LMS adaptive FIR filter architecture without adaptation delay," IEEE Trans. Signal Process., vol. 46, no. 3, pp. 775-779, Mar. 1998.

[168] B. Raghothaman, D. A. Linebarger, and D. Begusic, "A new method for low rank transform domain adaptive filtering," IEEE Trans. Signal Process., vol. 48, no. 4, pp. 1097-1109, May 2000.
[169] I. A. Hemadeh, K. Satyanarayana, M. El-Hajjar et al., "Millimeterwave communications: Physical channel models, design considerations, antenna constructions, and link-budget," IEEE Commun. Surveys Tuts., vol. 20, no. 2, pp. 870-913, Second Quart. 2018.

[170] D. DiSanto, T. Shirley, and R. Shimon, "Technology options for mmwave test and measurement equipment," in Proc. IEEE Compound Semiconductor Integrated Circuit Symposium, Miami, FL, USA, Oct. 2017, pp. 1-6.

[171] M. Toyoshima, Y. Takayama, T. Takahashi et al., "Ground-to-satellite laser communication experiments," IEEE Aerosp. Electron. Syst. Mag, vol. 23 , no. 8, pp. 10-18, Aug. 2008.

[172] H. Han, J. Yuan, and J. Tong, "Terahertz band communication systems: Challenges, novelties and standardization efforts," Journal of Computer and Communications, vol. 3, pp. 61-65, Mar. 2015.

[173] R. Zhang, K. Yang, Q. Abbasi et al., "Analytical characterisation of the terahertz in-vivo nano-network in the presence of interference based on TS-OOK communication scheme," IEEE Access, vol. 5, pp. 10172 10181 , Jun. 2017.

[174] K. Tekbiyik, A. R. Ekti, G. K. Kurt et al., "Terahertz band communication systems: Challenges, novelties and standardization efforts," Phys. Commun., vol. 35, pp. 1-18, May 2019.

[175] K. Tekbiyik, A. R. Ekti, G. K. Kurt et al., "A holistic investigation of terahertz propagation and channel modeling toward vertical heterogeneous networks," IEEE Commun. Mag., vol. 58, no. 11, pp. 14-20, Nov. 2020.

[176] J. N. Pelton, S. Madry, and S. Camacho-Lara, New Millimeter, Terahertz, and Light-Wave Frequencies for Satellite Communications. Springer International Publishing, Jan. 2017.

[177] Q. Wu, C. Lin, B. Lu et al., "A $21 \mathrm{~km} 5$ Gbps real time wireless communication system at $0.14 \mathrm{THz}$," in Proc. International Conference on Infrared, Millimeter, and Terahertz Waves, Cancun, Mexico, Sep. 2017, pp. 1-2.

[178] C. Wang, B. Lu, C. Lin et al., " $0.34 \mathrm{THz}$ wireless link based on highorder modulation for future wireless local area network applications," IEEE Trans. Terahertz Sci. Technol., vol. 4, no. 1, pp. 75-85, Jan. 2014.

[179] I. Kallfass, F. Boes, T. Messinger et al., "64 Gbit/s transmission over $850 \mathrm{~m}$ fixed wireless link at $240 \mathrm{GHz}$ carrier frequency," J. Infrared. Millim. Terahertz Waves, vol. 36, pp. 221-233, Feb. 2015.

[180] S. Moghadami, F. Hajilou, P. Agrawal et al., "A $210 \mathrm{GHz}$ fullyintegrated OOK transceiver for short-range wireless chip-to-chip communication in $40 \mathrm{~nm}$ CMOS technology," IEEE Trans. Terahertz Sci. Technol., vol. 5, no. 5, pp. 737-741, Sep. 2015.

[181] Z. Chen, B. Zhang, Y. Zhang et al., " $220 \mathrm{GHz}$ outdoor wireless communication system based on a schottky-diode transceiver," IEICE Electronics Express, vol. 13, no. 9, pp. 20160282-20160282, May 2016.

[182] V. Vassilev, Z. S. He, S. Carpenter et al., "Spectrum efficient D band communication link for real-time multi-gigabit wireless transmission," in Proc. IEEE International Microwave Symposium, Philadelphia, PA, USA, Aug. 2018, pp. 1523-1526.

[183] C. Castro, R. Elschner, J. Machado et al., "Ethernet transmission over a $100 \mathrm{~Gb} / \mathrm{s}$ real-time terahertz wireless link," in Proc. IEEE Globecom Workshops, Waikoloa, HI, USA, Dec. 2019, pp. 1-5.

[184] X. Li, J. Yu, L. Zhao et al., " $132 \mathrm{~Gb} / \mathrm{s}$ photonics-aided single-carrier wireless terahertz-wave signal transmission at $450 \mathrm{GHz}$ enabled by 64QAM modulation and probabilistic shaping," in Proc. Optical Fiber Communications Conference and Exhibition, San Diego, CA, USA, Apr. 2019, pp. 1-3.

[185] W. Zhou, L. Zhao, J. Zhang et al., " $135 \mathrm{GHz}$ D band 60 Gbps PAM- 8 wireless transmission employing a joint DNN equalizer with BP and CMMA," Journal of Lightwave Technology, vol. 38, no. 14, pp. 35923601, Jul. 2020

[186] Y. Feng, B. Zhang, C. Zhi et al., "A 20.8 Gbps dual-carrier wireless communication link in $220 \mathrm{GHz}$ band," China Commun., vol. 18, no. 5, pp. 210-220, May 2021.

[187] K. Liu, S. Jia, S. Wang et al., "100 Gbit/s THz photonic wireless transmission in the $350 \mathrm{GHz}$ band with extended reach," IEEE Photon. Technol. Lett., vol. 30, no. 11, pp. 1064-1067, Apr. 2018.

[188] Z. Lu, S. Wang, W. Li et al., " $26.8 \mathrm{~m} 350 \mathrm{GHz}$ wireless transmission of beyond $100 \mathrm{Gbit} / \mathrm{s}$ supported by THz photonics," in Proc. Asia Communications and Photonics Conference, Chengdu, China, Nov. 2019, p. M4D.6.

[189] I. Dan, G. Ducournau, S. Hisatake et al., "A terahertz wireless communication link using a superheterodyne approach," IEEE Trans. Terahertz Sci. Technol., vol. 10, no. 1, pp. 32-43, Jan. 2020. 
[190] H. Hamada, T. Tsutsumi, H. Matsuzaki et al., "300 GHz band 120 $\mathrm{Gb} / \mathrm{s}$ wireless front-end based on InP-HEMT PAs and mixers," IEEE J. Solid-State Circuits, vol. 55, no. 9, pp. 2316-2335, Jul. 2020.

[191] I. Dan, P. Szriftgiser, E. Peytavit et al., "A $300 \mathrm{GHz}$ wireless link employing a photonic transmitter and an active electronic receiver with a transmission bandwidth of $54 \mathrm{GHz}$," IEEE Trans. Terahertz Sci. Technol., vol. 10, no. 3, pp. 271-281, Mar. 2020.

[192] H. Kaushal and G. Kaddoum, "Optical communication in space: Challenges and mitigation techniques," IEEE Commun. Surveys Tuts., vol. 19, no. 1, pp. 57-96, First Quart. 2017.

[193] M. Toyoshima, "Trends in satellite communications and the role of optical free-space communications," Journal of Optical Networking, vol. 4, no. 6, pp. 300-311, 2005.

[194] A. U. Chaudhry and H. Yanikomeroglu, "Free space optics for nextgeneration satellite networks," IEEE Consum. Electron. Mag., vol. 10, no. 6, pp. 21-31, Nov. 2021.

[195] Q. Chen, G. Giambene, L. Yang et al., "Analysis of inter-satellite link paths for LEO mega-constellation networks," IEEE Trans. Veh. Technol., vol. 70, no. 3, pp. 2743-2755, Mar. 2021.

[196] A. U. Chaudhry and H. Yanikomeroglu, "Laser intersatellite links in a starlink constellation: A classification and analysis," IEEE Veh. Technol. Mag., vol. 16, no. 2, pp. 48-56, Apr. 2021.

[197] T. Tolker-Nielsen and G. Oppenhauser, "In-orbit test result of an operational optical intersatellite link between ARTEMIS and SPOT4, SILEX," in Proc. Free-Space Laser Communication Technologies, vol. 4635, San Jose, CA, USA, Apr. 2002, pp. 1-15.

[198] T. Jono, Y. Takayama, K. Shiratama et al., "Overview of the interorbit and the orbit-to-ground laser communication demonstration by OICETS," in Proc. Free-Space Laser Communication Technologies XIX and Atmospheric Propagation of Electromagnetic Waves, vol. 6457, San Jose, CA, USA, Mar. 2007, pp. 9-18.

[199] M. Gregory, F. Heine, H. Kämpfner et al., "TESAT laser communication terminal performance results on 5.6Gbit coherent inter satellite and satellite to ground links," in Proc. International Conference on Space Optics, E. Armandillo, B. Cugny, and N. Karafolas, Eds., vol. 10565, Rhodes Island, Greece, Nov. 2017, pp. 324-329.

[200] M. Toyoshima, T. Fuse, D. R. Kolev et al., "Current status of research and development on space laser communications technologies and future plans in NICT," in 2015 IEEE International Conference on Space Optical Systems and Applications, Oct. 2015, pp. 1-5.

[201] T. Wang, P. Lin, F. Dong et al., "Progress and prospect of space laser communication technology," Strategic Study of Chinese Academy of Engineering, vol. 22, no. 3, pp. 92-99, May 2020.

[202] H. Zech, F. Heine, D. Tröndle et al., "LCT for EDRS: LEO to GEO optical communications at 1.8 Gbps between Alphasat and Sentinel 1a," in Proc. Advanced Free-Space Optical Communication Techniques and Applications, vol. 9647, Toulouse, France, Oct. 2015, pp. 85-92.

[203] B. V. Oaida, M. J. Abrahamson, R. J. Witoff et al., "OPALS: An optical communications technology demonstration from the international space station," in Proc. IEEE Aerospace Conference, Big Sky, MT, USA, May 2013, pp. 1-20.

[204] A. Carrasco-Casado, H. Takenaka, D. Kolev, et al., "LEO-to-ground optical communications using sota (small optical transponder)-payload verification results and experiments on space quantum communications," Acta Astronaut., vol. 139, pp. 377-384, Oct. 2017.

[205] W. Chen, L. Sun, i, K. Xie et al., "5.12Gbps optical communication link between LEO satellite and ground station," in Proc. IEEE International Conference on Space Optical Systems and Applications, Naha, Japan, Nov. 2017, pp. 260-263.

[206] T. S. Rose, D. W. Rowen, S. LaLumondiere et al., "Optical communications downlink from a $1.5 \mathrm{U}$ Cubesat: OCSD program," in Proc. International Conference on Space Optics, Z. Sodnik, N. Karafolas, and B. Cugny, Eds., vol. 11180, Chania, Greece, Jul. 2019, pp. 201212.

[207] H. Takenaka, A. Carrasco-Casado, M. Fujiwara et al., "Satelliteto-ground quantum-limited communication using a $50-\mathrm{kg}$-class microsatellite," Nature photonics, vol. 11, no. 8, pp. 502-508, Aug. 2017.

[208] R. Zhang, W. Zhang, X. Zhang et al., "Research status and development trend of high earth orbit satellite laser relay links," Laser Optoelectronics Progress, vol. 58, no. 5, pp. 1-13, Mar. 2021.

[209] D. Calzolaio, F. Curreli, J. Duncan et al., "EDRS-C - the second node of the european data relay system is in orbit," Acta Astronaut., vol. 177, pp. 537-544, Dec. 2020.

[210] C. Xu, Y. Jin, L. Li et al., "Wireless transmission technology of satellite-terrestrial integration for 6G mobile communication," Journal of Electronics Information Technology, vol. 43, no. 1, pp. 28-36, Jan. 2021
[211] A. Carrasco-Casado, P. X. Do, D. Kolev et al., "Intersatellite-link demonstration mission between CubeSOTA (LEO CubeSat) and ETS9HICALI (GEO Satellite)," in Proc. IEEE International Conference on Space Optical Systems and Applications, Portland, OR, USA, Oct. 2019, pp. 1-5.

[212] H. Hauschildt, N. le Gallou, S. Mezzasoma et al., "Global quasi-realtime-services back to Europe: EDRS Global," in Proc. International Conference on Space Optics, vol. 11180, Chania, Greece, Oct. 2018, pp. 353-357.

[213] H. Hauschildt, C. Elia, A. Jones et al., "ESAs ScyLight programme: Activities and status of the high throughput optical network" HydRON"," in Proc. International Conference on Space Optics, vol. 11180, Chania, Greece, Oct. 2018, pp. 1-8.

[214] X. Li, J. Ma, S. Yu et al., "Investigation of optical intensity fluctuation in the presence of satellite vibration for intersatellite optical communications," in Proc. International Conference on Computer Science and Network Technology, vol. 1, Harbin, China, Dec. 2011, pp. 65-67.

[215] Q. Yang, L. Tan, and J. Ma, "Doppler characterization of laser intersatellite links for optical LEO satellite constellations," Opt. Commun., vol. 282 , no. 17 , pp. 3547-3552, Sep. 2009.

[216] M. Cannon, A. Keller, and M. Wirthlin, "Improving the effectiveness of TMR designs on FPGAs with SEU-aware incremental placement," in Proc. IEEE International Symposium on Field-Programmable Custom Computing Machines, Boulder, CO, USA, Sep. 2018, pp. 141-148.

[217] O. Gonçalves, G. Prenat, G. Di Pendina et al., "Nonvolatile runtimereconfigurable FPGA secured through MRAM-based periodic refresh," in Proc. IEEE International Memory Workshop, Monterey, CA, USA, Aug. 2013, pp. 170-173.

[218] F. L. Kastensmidt, L. Carro, and R. A. da Luz Reis, Fault-tolerance techniques for SRAM-based FPGAs. Springer, 2006, vol. 1.

[219] M. Yin, "SEU-tolerant design of SRAM FPGA for space use," Spacecraft Environ. Eng., vol. 28, no. 6, Dec. 2011.

[220] N. Hosseinidehaj, Z. Babar, R. Malaney et al., "Satellite-based continuous-variable quantum communications: State-of-the-art and a predictive outlook," IEEE Commun. Surveys Tuts., vol. 21, no. 1, pp. 881-919, First Quart. 2019.

[221] J. Yin, Y. Cao, and Y. Li, "Satellite-based entanglement distribution over 1200 kilometers," Science, vol. 356, no. 6343, pp. 1140-1144, Jun. 2017.

[222] G.-L. Long and X.-S. Liu, "Theoretically efficient high-capacity quantum-key-distribution scheme," Physical Review A, Feb. 2002. [Online]. Available: http://dx.doi.org/10.1103/PhysRevA.65.032302

[223] F.-G. Deng, G. L. Long, and X.-S. Liu, "Two-step quantum direct communication protocol using the Einstein-Podolsky-Rosen pair block," Physical Review A, Oct. 2003. [Online]. Available: https://link.aps.org/doi:10.1103/PhysRevA.68.042317

[224] F.-G. Deng and G. L. Long, "Secure direct communication with a quantum one-time pad," Physical Review A, May 2004. [Online]. Available: https://link.aps.org/doi/10.1103/PhysRevA.69.052319

[225] F. Yan and X. Zhang, "A scheme for secure direct communication using EPR pairs and teleportation," The European Physical Journal $B$-Condensed Matter and Complex Systems, vol. 41, no. 1, pp. 75-78, Sep. 2004.

[226] C. Wang, F.-G. Deng, Y.-S. Li et al., "Quantum secure direct communication with high-dimension quantum superdense coding," Physical Review A, Apr. 2005. [Online]. Available: https://doi.org/10. 1103/PhysRevA.71.044305

[227] Z. Zhou, Y. Sheng, P. Niu et al., "Measurement-device-independent quantum secure direct communication," Science China Physics, Me chanics \& Astronomy, vol. 63, no. 3, pp. 1-6, Dec. 2019.

[228] A. Huang, S. Barz, E. Andersson et al., "Implementation vulnerabilities in general quantum cryptography," New Journal of Physics, Oct. 2018. [Online]. Available: https://doi.org/10.1088/1367-2630/aade06

[229] D. Chandra, A. S. Cacciapuoti, M. Caleffi et al., "Direct quantum communications in the presence of realistic noisy entanglement," IEEE Trans. Commun., Oct. 2021. [Online]. Available: 10.1109/TCOMM. 2021.3122786

[230] Z. Sun, L. Song, Q. Huang et al., "Toward practical quantum secure direct communication: A quantum-memory-free protocol and code design," IEEE Trans. Commun., vol. 68, no. 9, pp. 5778-5792, Jul. 2020 .

[231] Q. Y. Yu, H. C. Lin, and H. H. Chen, "Intelligent radio for next generation wireless communications: An overview," IEEE Wireless Commun., vol. 26, no. 4, pp. 94-101, Aug. 2019.

[232] L. Li, Z. Zhang, K. Xue et al., "AI-aided downlink interference control in dense interference-aware drone small cells networks," IEEE Access, vol. 8, pp. $15110-15122$, Jan. 2020. 
[233] Z. Yang, K. Yang, L. Lei et al., "Blockchain-based decentralized trust management in vehicular networks," IEEE Internet Things J., vol. 6, no. 2, pp. 1495-1505, Mar. 2019.

[234] T. Huang, W. Yang, J. Wu et al., "A survey on green 6G network: Architecture and technologies," IEEE Access, vol. 7, pp. 175758 175768 , Dec. 2019.

[235] W. Li, Z. Su, R. Li et al., "Blockchain-based data security for artificial intelligence applications in 6G networks," IEEE Netw., vol. 34, no. 6, pp. 31-37, Nov/Dec. 2020.

[236] S. A. Surdi, "Space situational awareness through blockchain technology," J. Space Saf. Eng., vol. 7, no. 3, pp. 295-301, Sep. 2020.

[237] S. Fu, J. Gao, and L. Zhao, "Integrated resource management for terrestrial-satellite systems," IEEE Trans. Veh. Technol., vol. 69, no. 3, pp. 3256-3266, Mar. 2020.

[238] R. Han, L. Bai, J. Liu et al., "Blockchain-based GNSS spoofing detection for multiple UAV systems," J. Commun. Netw., vol. 4, no. 2 , pp. 81-88, Jun. 2019.

[239] H. Hashida, Y. Kawamoto, and N. Kato, "Intelligent reflecting surface placement optimization in air-ground communication networks toward 6G," IEEE Wireless Commun., vol. 27, no. 6, pp. 146-151, Dec. 2020.

[240] G. Pan, J. Ye, J. An et al., "Full-duplex enabled intelligent reflecting surface systems: Opportunities and challenges," IEEE Wireless Commun., vol. 28, no. 3, pp. 122-129, Jun. 2021.

[241] S. Gong, X. Lu, D. T. Hoang et al., "Toward smart wireless communications via intelligent reflecting surfaces: A contemporary survey," IEEE Commun. Surveys Tuts., vol. 22, no. 4, pp. 2283-2314, Fourth Quart. 2020.

[242] J. Lee, W. Shin, and J. Lee, "Performance analysis of IRS-assisted LEO satellite communication systems," in Proc. International Conference on Information and Communication Technology Convergence, Jeju Island, South Korea, Dec. 2021, pp. 323-325.

[243] B. Matthiesen, E. Björnson, E. De Carvalho et al., "Intelligent reflecting surface operation under predictable receiver mobility: A continuous time propagation model," IEEE Wireless Commun. Lett., vol. 10, no. 2, pp. 216-220, Feb 2021.

[244] X. Yu, D. Xu, and R. Schober, "Enabling secure wireless communications via intelligent reflecting surfaces," in Proc. IEEE Glob. Commun. Conf., Waikoloa, HI, USA, Dec. 2019, pp. 1-6.

[245] X. Yu, D. Xu, Y. Sun et al., "Robust and secure wireless communications via intelligent reflecting surfaces," IEEE J. Sel. Areas Commun., vol. 38 , no. 11 , pp. 2637-2652, Nov. 2020 